\title{
Every entangled stuff has its own avatar
}

\author{
Mario Mastriani \\ Quantum Communications Lab, Qubit Reset LLC, 2875 NE 191, suite 801, Aventura, FL 33180, USA
}

\author{
ORCID Id: 0000-0002-5627-3935 \\ mmastri@qubitreset.com
}

\begin{abstract}
During the last century, entanglement was the bone of contention between the two main pillars of Physics: General Relativity (GR) and Quantum Mechanics (QM). This began in 1935 with the Einstein-Podolsky-Rosen paradox (EPR paradox) which concluded that although Quantum Mechanics is not wrong, it is an incomplete theory to represent physical reality. In this paper it is demonstrated that some byproducts resulting from entanglement, which we will call avatars, act as a hinge that link both theories making the completeness of QM clear. Moreover, a thorough analysis of the non-locality of this effect will be carried out while demonstrating that entanglement is an instantaneous phenomenon, and that it does not require the use of a superluminal signaling for this purpose. Finally, the avatars will also appear in each wormhole resulting from an entanglement process (WREP) demonstrating that they are traversable with an equivalent path of null length which can be crossed in a null time with all that this implies in Quantum Communications.
\end{abstract}

Keywords-Entanglement; EPR Paradox; ER = EPR conjecture; GR = QM; Locality; Theory of Everything; Quantum Communications.

PACS numbers: 03.67.Hk, 03.65.Ud, 03.67.-a, 03.67.Lx, 03.65.Yz, 03.65.Yz, 03.67.Bg, 04.62.+v, 04.70.-s, 04.70.Dy, 04.20.-q, 03.67.Mn, 03.65.-w

\begin{abstract}
Prologue - This paper attempts to evaluate the viability of the GR=QM conjecture. Therefore, it has been completely developed outside of String Theory for reasons that will be understood during the reading of this work. It has also been stripped of all symbolic logic in order to facilitate physical expression over mathematical symbolism and thus achieve the greatest audience of possible readers.
\end{abstract}

\section{Introduction}

Quantum entanglement [1-3] has turned out to be one of the hottest frontiers between the two greatest exponents of Modern Physics: the Theory of Relativity (TR) [4] and Quantum Mechanics (QM) [5-9]. Another controversial border, undoubtedly is constituted by the study of black holes (BH) in general and its supposedly interior in particular [10-13]. We said supposedly because there is a relatively new analysis by Almheiri-Marolf-Polchinski-Sully (AMPS) [14] which tries to resolve the controversy between the complementarity and firewalls in a $\mathrm{BH}$ concluding that these do not have a deep interior but that they end in a firewall [14] necessary to avoid violating the entanglement monogamy [1-3] between the resulting particles of a Hawking's radiation [11, 13]. Both controversies are consistent with the great affirmation made in the 70s by the Prof. Stephen Hawking [15]: "the information is lost inside a BH". The consequences of this statement -if so- would be deadly for QM and inadmissible for a large number of physicists, given that if the information is lost inside a $\mathrm{BH}$, then the QM loses its main attribute: the reversibility and therefore it dies. This has led to a real intellectual duel between them, which became known as "the war of BHs" [16] in relation to the so-called "BH Information Paradox" [16]. So far, several theories and conjectures have been developed to resolve this controversy. In this context, the ER = EPR conjecture [17-19] appears with the intention of giving an answer to the AMPS problem of firewalls [14].

On the other hand, the failure of the GR to approach the study of particles and subatomic particles is similar to the failure of the QM when facing the planetary systems. This is highlighted in the BHs as in no other scenario, driving away all hope for an early Theory of Everything (TOE) [20]. 
A similar situation arises when trying to unify the four fundamental forces of the universe [20]: the electromagnetism, the strong nuclear force, the weak nuclear force and gravity. The first three are insignificant at the planetary level (except for the magnetic shield that protects us from solar radiation) while the last one is insignificant at the level of particles and subatomic particles.

A delicate dilemma has been installed since 1935 with the appearance of the EPR paradox paper [21] in relation to the instantaneous nature of the entanglement, which according to the GR [4] is only reserved for local phenomena. Thus, Einstein understood that the only way to justify such behavior by this phenomenon is through local hidden variables, which unequivocally determined and conditioned any experiment related to it. Einstein proposed a thought experiment (in German: gedankenversuch) which involves a pair of gloves that are separated into two corresponding boxes and distributed to two distant people (Alice and Bob) without knowing initially which glove each one received. When Alice opens her box and finds a right glove, we are instantly certain that Bob has the left one. The problem is that the entanglement does not work that way, since in each box there will be a glove that is right and left at the same time and once one of the boxes is opened (that is, we had applied quantum measurement [22]) then and only then, if the glove in question is right, the other one instantaneously, and only at that precise moment, becomes the left one and not before; therefore, there is no preexisting conditioning of a local character as Einstein suggested. In fact, there are not even two gloves but a combination of both of them distributed or shared by both boxes. However, and based on a lack of knowledge in the profound nature of the entanglement and its eventual collision with the GR, Einstein and his colleagues inferred that the QM was not wrong but incomplete. The current position is more tolerant, yet it is far from permissive given that although the conflict between the instantaneous nature of entanglement and the postulates of the GR is evident, physicists recognize both positions but with the proviso that useful information cannot be transmitted to a speed greater than that of light [23-29].

For many physicists, the fact that you cannot transmit useful information through an instantaneous link based on entanglement is a cold water cloth on an infected wound, that is, it is a rare and questionable palliative that nothing explains and much less solves. In fact, strictly speaking and according to them, regardless of whether useful information can be transmitted or not, no non-local phenomenon (such as entanglement) can be instantaneous. Formally, and leaving aside our insatiable technological appetite for trying to communicate two infinitely distant points in an instantaneously form, lies the fact that we are still not able to explain how when Alice opens her box the state of itself is completely defined and therefore the state of Bob's box is also defined in an unequivocally and instantaneously way from the other side of the universe for a non-local phenomenon. This is something more than counter-intuitive or non-intuitive, it is something which is directly absent or completely wrong in the hypotheses that are sustained. For this reason, a pivotal work emerges in the history of QM due to John Bell [30] and published in 1964, which raises an experimental possibility to definitively derive the question of locality or non-locality of entanglement. In this paper, an inequality is posed, which, if violated, would prove the non-locality of QM, which has been experimentally verified in laboratories all over the world [31,32] since then. The theorem developed by Bell tells us that if its inequality is violated by the QM, then there are no local hidden variables associated with the entanglement, but says nothing about hidden non-local variables. On this hypothesis, Bohm develops a theory based on hidden nonlocal variables in order to explain the entanglement [33].

After Bell's work an improvement of his theorem appeared due to Clauser-Horne-Shimony-Holt (CHSH) [34] which is used today. Both the Bell's version and the CHSH's version are not exempt from critics, given that they are reproached in the following points:

- the existence of loopholes $[31,32]$ which distorts the experimental results,

- the existence of hidden hypothesis [35, 36] which sows a seed of doubt about the rigor of the theorem, and,

- the fact of leaving aside the most conspicuous icon of the locality, which is undoubtedly the Euclidean distance, given that Bell's theorem (as well as CHSH [34]) only speaks of angles and projections [30]. 
This last criticism has been practiced in a much more reserved way than the previous ones although, to date, there is no published paper that supports it. However, in his time, this criticism seems to have reached John Bell's ears since he himself made a definition per se of the locality based on the past light cones of the individual particles and the overlapping of such said cones for entangled particles and that it will be developed in more detail in the next section. Notwithstanding the above, Bell also does not explain how a non-local phenomenon can be instantaneous at infinitely long distances.

Evidently, any new contribution that helps to clarify, even if not in part, the doubts and apparent contradictions raised in this section will be very welcome. This is the motivation that gave rise to this work.

Finally, the necessary tools for the treatment of this paper are exposed in $\S 2$. $\S 3$ is devoted to analyzing the degree of subsystems' correlations. Attributes and particular characteristics of avatars are treated in $\$ 4$. $\S 5$ is devoted to the generalization of the concepts developed in $\S 3$. In $\S 6$, we will demonstrate the consequences of the avatars' existence. Finally, conclusions and future works are made in $\S 7$.

\section{Setup}

The doubts of the scientific community about the rigor of Bell's Theorem to demonstrate that entanglement is a non-local phenomenon are explored in this section. The analysis and study of such doubts gave origin to the new tool called avatar, as well as the tools necessary for its manifestation.

\subsection{Locality, past light cones and the Bell's theorem}

Much has been written about locality and non-locality [37-41], past light cones and space-like separations [42-46] and the influence domain of a wave-function for an isolated particle as well as for an EPR pair [1-3]. Although it is a huge amount of analysis, it has left aside a phenomenon that is hard to assimilate and consists in the instantaneous nature of the entanglement [1]. Let us illustrate this with the following example: suppose we have two entangled particles [2] like those in Figure 2.1, where the arrow pointing up means spin-up and the arrow pointing down represents spin-down [1-3]. The colours represent which subsystem each particle belongs to: red for subsystem A (Alice) and green for subsystem B (Bob). We can then give the entanglement any of the two interpretations presented in the upper part of the figure above the light green line, i.e., the one on the left or the one on the right.

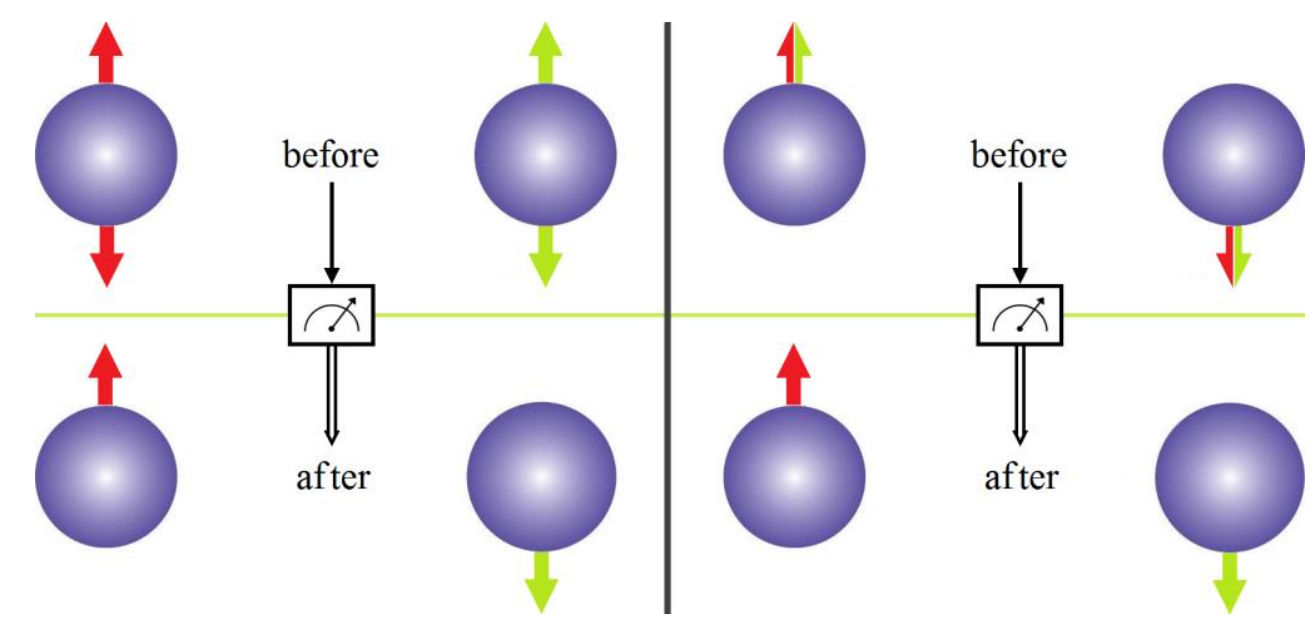

Figure 2.1 To the left of the vertical black line and above the horizontal green line we have a way of representing an EPR pair. After the quantum measurement of one of the elements of the pair, its spin is defined; therefore, the spin of its counterpart is defined instantly, too. We can see that at the bottom of the figure. To the right of the vertical black line and above the horizontal green line we have another way of representing an EPR pair, however, when we perform a quantum measurement we obtain the same result as in the previous case, which can be observed in the lower part of the figure. 
In both cases each element of the EPR pair shares a non-separable combination of the original spins. In the image on the left, we do not know if both of them (Alice and Bob) have a spin-up or a spindown in their hands, while in the image on the right, we do not know if both spins: spin-up and spindown belong to Alice or Bob. Another important fact is to consider each element of the EPR pair in diametrically opposite extremes of the universe. If now we perform a quantum measurement [22] on any of them, then we will find the scenario established in the lower part of Figure 2.1, where both elements are unequivocally defined at the same instant, i.e., instantaneously. But, how is it possible that there is such coordination between elements separated by galactic distances? Now, if both elements of the pair were stuck side by side sharing the same location, that is, both were in the same point, there would be no problem. This is represented without problems by the equation (2.1)

$d=c t=310^{8} \mathrm{~m} / \mathrm{sec} 0 \mathrm{sec}=0 \mathrm{~m}$

where $d$ is the distance between both elements of the EPR pair, $c$ is the speed of light and $t$ is the elapsed time for the influence of the measurement to go from one element of the pair to the other one. Now, being the entanglement instantaneous $t=0$, and being $c$ a finite number expressed in meter per seconds, the distance $d$ will result equal to zero too, as equation (2.1) shows us. Instead, if we send an element of the pair EPR to Alpha Centauri and the other one we leave it on the Earth and we make a measurement on the latter, so that the influence of the measurement arrives instantly at Alpha Centauri, then, a speed like the following will be needed:

$v=\frac{d}{t}=\frac{4367 \text { light }- \text { years }}{0 \text { sec }}=\infty=\infty c \gg c$

It is evident that we have a problem because the TR tells us that nothing can travel faster than light, so either entanglement is not instantaneous or there is something that we may not see. The truth of this matter, and the way we see it, goes beyond these two aspects: the experimental implementation of Bell's theorem without loopholes [47], and whether we should confirm the non-local nature of entanglement. What is relevant here is to understand how nature can send messages instantaneously between two diametrically opposed points of the universe. At this point, it does not even matter if the mentioned quality can be used by the man to send useful information or not, since what we are trying to understand is: how does nature work? After all, that is what Physics is all about. But, since we are convinced that the entanglement is instantaneous, then it is evident that there is something we are not acknowledging. We will try to find out what it is, although unfortunately Bell will help us little or nothing in this difficult task. The reasons for this claim lies in the fact that Bell says nothing about the instantaneity of entanglement. Besides, Bell's definition of locality based on local beables [48] does not apply to any of these: arbitrary theories and non-Markovian theories. For this reason, robustness and generality are lost when we try to analyze a problem as complex as the instantaneity of the entanglement with that tool. In addition, it is evident that the mere notion of locality from past light cones that are overlapping is very weak to face such a challenge. However, if we leave the instantaneity of entanglement aside for a moment and return to the notion of Bell's locality with projection on his famous theorem [30] we also encounter problems.

In order to better understand this last observation, we propose the following mental experiment based on Figure 2.2 without caring, initially, whether or not it has loopholes. If we start with the EPR pair of the extremes, i.e., the EPR located in positions A and B, which are 10.8 kilometers apart from each other [49], it is clear that the experimental evidence obtained from the application of Bell's Theorem [30] results in the non-locality of both elements (EPR pair). If we now approach both elements of the pair at a distance of-say-12 meters between them [32] (it goes without saying that the figure does not respect the distance scales established here), the new positions will be $\mathrm{A}^{\prime}$ and $\mathrm{B}^{\prime}$. Thus, when we apply the theorem again in an experimental way, the result will be identical to the previous one, that is, the phenomenon is still non-local. But, if we now put together both elements of the pair in such a way that they touch each other (and in fact, so do the respective measuring instruments), the new positions coincide (A" $\equiv$ B") and clearly both spins will be local among them under the same past light cone. 


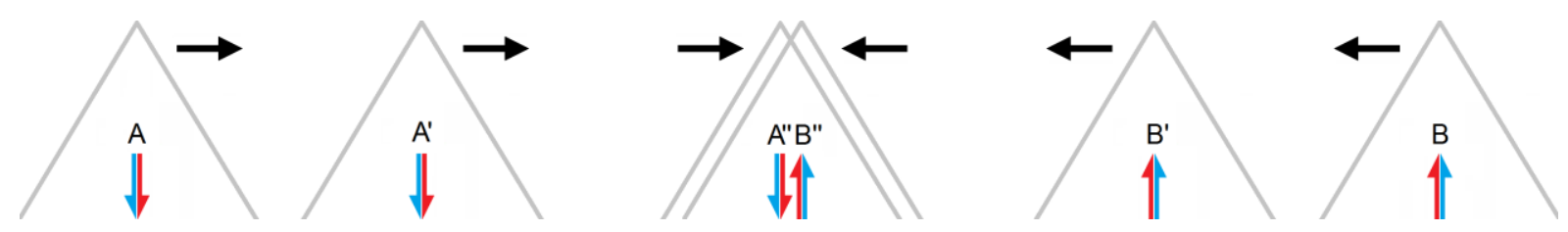

Figure 2.2 Mental experiment which begins at the extremes A and B, where the experimental implementation of Bell's Theorem indicates that the EPR pair is non-local. Similar results can be reached when both EPR elements are in position A' and B', much closer to each other than in the previous case. In the last case, both elements of the EPR pair are in the same location, i.e., A" = B", under the same past light cone, however the experimental implementation of Bell's Theorem continues saying that both elements are non-local when it is evident that they are mutually local.

However, the experimental application of Bell's theorem will yield the same results as in the two previous cases, that is, it will tell us that they are non-local. It is evident that there is a contradiction in this last case. So, both Bell [30] and CHSH [34] do not make any contribution to clarify anything about the instantaneity of entanglement but also do not give convincing results, or the mentioned results are not given in circumstances that assure the entire scientific community about the non-locality of entanglement, even in the case when experimental implementations without loopholes [47] are considered. Therefore, based on Bell's Theorem, we cannot be $100 \%$ sure whether or not the entanglement includes the two main attributes necessaries to be used with total success in quantum communications: instantaneity and non-locality. However, one thing that Bell's Theorem does confirm is that locality means that physical influences cannot propagate faster than light, which we already knew due to GR.

Returning to equations (2.1) and (2.2) and considering that the speed of light is finite, an instantaneous interchange of information between two observers can only occur when the equivalent Euclidean distance between them is zero. On the other hand, if the distance between both observers is not null and even though we pretend that the interchange is instantaneous, then the conclusion is inadmissible since the interchange speed should be infinite. With these extreme examples, we tried to establish that the discussion should go beyond whether or not the observers are local, since they are not local enough to explain the instantaneity of entanglement. This comment responds to the fact that nowadays all the efforts are oriented to improve the experimental implementations of Bell's theorem [50], as if by testing it, we would finally be sure this is something positive for Physics.

Notwithstanding the foregoing, it is evident that entanglement is a non-local and instantaneous phenomenon, independently of the possibility of being able to use a link based on it to send useful information or not.

Summing up, our main commitment should be with the formalization and thoroughly understanding of this fascinating phenomenon called entanglement. Or in other words, we need the right tools to test what we know (or believe we know) so as not to have any questions about it.

\subsection{Quantum entanglement and its measurement}

For pure states, that is, states on the Bloch's sphere [51-53] of Figure 2.3, any wave-function

$|\psi\rangle=\alpha|0\rangle+\beta|1\rangle$

arises from the superposition of the so-called Computational Basis States (CBS) or qubit basis states $\{|0\rangle,|1\rangle\}$, which are located at the poles of the already mentioned sphere with $|\alpha|^{2}+|\beta|^{2}=1$, such that $\alpha \wedge \beta \in \mathbb{C}$. Strictly, the complete wave-function will be

$$
|\psi\rangle=e^{i \gamma}\left(\cos \frac{\theta}{2}|0\rangle+e^{i \phi} \sin \frac{\theta}{2}|1\rangle\right)=e^{i \gamma}\left(\cos \frac{\theta}{2}|0\rangle+(\cos \phi+i \sin \phi) \sin \frac{\theta}{2}|1\rangle\right)
$$


where $0 \leq \theta \leq \pi, 0 \leq \phi<2 \pi$ [51]. However, we can ignore the factor of $e^{i \gamma}$ out the front, because it has no observable effects [52], and for that reason we can effectively write

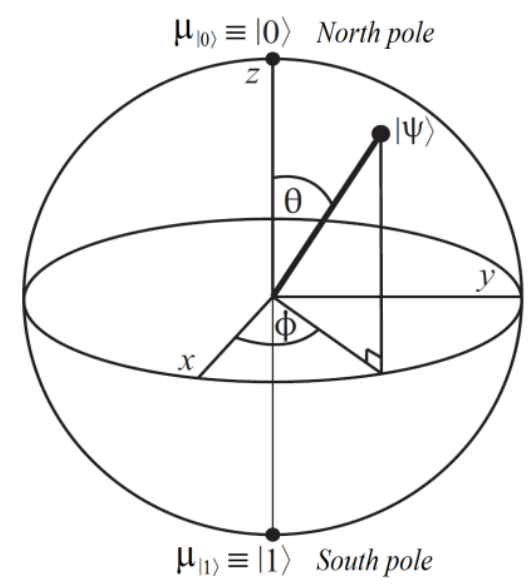

Figure 2.3 Bloch's Sphere.

$|\psi\rangle=\cos \frac{\theta}{2}|0\rangle+e^{i \phi} \sin \frac{\theta}{2}|1\rangle$

with $\alpha=\cos (\theta / 2)$ and $\beta=e^{i \phi} \sin (\theta / 2)$, being then equal to the equation (2.4). The numbers $\theta$ and $\phi$ define a point on the unit three-dimensional sphere, as shown in Figure 2.3.

Now, we can represent the mentioned poles of different ways:

$$
\begin{aligned}
& \left(\text { Spin up }=|\uparrow\rangle=|0\rangle=\left[\begin{array}{l}
1 \\
0
\end{array}\right]=\text { North pole }\right) \equiv \mu_{|0\rangle} \\
& \left(\text { Spin down }=|\downarrow\rangle=|1\rangle=\left[\begin{array}{l}
0 \\
1
\end{array}\right]=\text { South pole }\right) \equiv\left(\mu_{|1\rangle}=-\mu_{|0\rangle}\right)
\end{aligned}
$$

where $\mu_{|0\rangle}=1$, being $\mu_{|0\rangle}$ and $\mu_{|1\rangle}$ the scalar versions of the spin up $|0\rangle$ and the spin down $|1\rangle$, respectively, in such a way that the complete spin is conserved, i.e., $\mu_{|0\rangle}+\mu_{|1\rangle}=0$, similarly is the case when a spinless particle decays in two new entangled particles with opposite spins $[1,2,51]$. We can see them in both poles of the Figure 2.3. The scalar notation based on $\mu$ is arithmetically more ductile than the Dirac's notation [51] based on <bra| and |ket> and allows us to work better with magnitudes and signs which will be fundamental in the discovery of the avatars. In fact, in this notation, we associate the sign of $\mu$ with the mentioned spin orientation or polarization of the entangled particles. These $\mu$ 's will have as much generality as the Dirac's notation in regards to its validity for all types of particles. Therefore, in this paper, the letter $\mu$ will represent this concept, exclusively, and does not necessarily mean muon. Moreover, and based on CBS, we can define another basis, which will be very useful for the rest of this paper as well as for Quantum Information in general [51], and quantum teleportation and superdense coding in particular [1, 51, 54-60]. Taking into account the interaction of two subsystems $\mathrm{A}$ and $\mathrm{B}$, considering that their components are pure states and using their scalar versions here too,

$$
\begin{aligned}
& \left(\left|0^{A}\right\rangle \otimes\left|0^{B}\right\rangle=\left|0^{A}\right\rangle\left|0^{B}\right\rangle=\left|0^{A}, 0^{B}\right\rangle=\left|0^{A} 0^{B}\right\rangle\right) \equiv\left(\mu_{\left|0^{A} 0^{B}\right\rangle}=\mu_{\left|0^{A, B}\right\rangle}^{2}\right) \\
& \left(\left|1^{A}\right\rangle \otimes\left|1^{B}\right\rangle=\left|1^{A}\right\rangle\left|1^{B}\right\rangle=\left|1^{A}, 1^{B}\right\rangle=\left|1^{A} 1^{B}\right\rangle\right) \equiv\left(\mu_{\left|1^{A} 1^{B}\right\rangle}=-\mu_{\left|1^{A, B}\right\rangle}^{2}\right)
\end{aligned}
$$


where $\otimes$ is the Kronecker's product, and $\mu_{\left|0^{A} 0^{B}\right\rangle}$ and $\mu_{\left|1^{A} 1^{B}\right\rangle}$ are the scalar version of $\left|0^{A} 0^{B}\right\rangle$ and $\left|1^{A} 1^{B}\right\rangle$, respectively, with $\mu_{\left|0^{A} 0^{B}\right\rangle}+\mu_{\left|1^{A} 1^{B}\right\rangle}=0$, i.e., here also the spin is conserved between $\mu_{\left|0^{A} 0^{B}\right\rangle}$ and $\mu_{\left|1^{A} 1^{B}\right\rangle}$, being their values +1 and -1 , respectively. The rule of the signs that support the equivalences and equalities seen so far is very simple:

$$
\begin{aligned}
& \mu_{|0\rangle}=-\mu_{|1\rangle} \\
& \mu_{|00\rangle}=-\mu_{|11\rangle} \\
& \mu_{|00\rangle}=\mu_{|0\rangle}^{2} \\
& \mu_{|11\rangle}=-\mu_{|1\rangle}^{2}
\end{aligned}
$$

Equations (2.9a) and (2.9b) were previously seen in relation to spin conservation, while equations (2.9c) and (2.9d) were seen to be highly intuitive, therefore, we will not go further into them. On the other hand, and being

$$
|00\rangle=\left[\begin{array}{l}
1 \\
0
\end{array}\right] \otimes\left[\begin{array}{l}
1 \\
0
\end{array}\right]=\left[\begin{array}{l}
1 \\
0 \\
0 \\
0
\end{array}\right], \quad|11\rangle=\left[\begin{array}{l}
0 \\
1
\end{array}\right] \otimes\left[\begin{array}{l}
0 \\
1
\end{array}\right]=\left[\begin{array}{l}
0 \\
0 \\
0 \\
1
\end{array}\right], \quad|01\rangle=\left[\begin{array}{l}
1 \\
0
\end{array}\right] \otimes\left[\begin{array}{l}
0 \\
1
\end{array}\right]=\left[\begin{array}{l}
0 \\
0 \\
1 \\
0
\end{array}\right], \quad|10\rangle=\left[\begin{array}{l}
0 \\
1
\end{array}\right] \otimes\left[\begin{array}{l}
1 \\
0
\end{array}\right]=\left[\begin{array}{l}
0 \\
1 \\
0 \\
0
\end{array}\right]
$$

we are going to use them to build the famous Bell's bases [1-3], with 2-qubit vectors, the combined Hilbert space will be $\mathrm{H}^{A \cup B}=\mathrm{H}_{2}^{A} \otimes \mathrm{H}_{2}^{B}$, and then we will have the following four vectors,

$$
\begin{aligned}
& \left|\beta_{00}\right\rangle=\left|\Phi^{+}\right\rangle=\frac{1}{\sqrt{2}}(|00\rangle+|11\rangle), \\
& \left|\beta_{01}\right\rangle=\left|\Psi^{+}\right\rangle=\frac{1}{\sqrt{2}}(|01\rangle+|10\rangle), \\
& \left|\beta_{10}\right\rangle=\left|\Phi^{-}\right\rangle=\frac{1}{\sqrt{2}}(|00\rangle-|11\rangle), \\
& \left|\beta_{11}\right\rangle=\left|\Psi^{-}\right\rangle=\frac{1}{\sqrt{2}}(|01\rangle-|10\rangle) .
\end{aligned}
$$

Moreover and considering that a maximally entangled pair emerges from a spinless particle, it is evident that the bases of the equation (2.11) must also comply with the conservation of the spin. Thus, for $\left|\beta_{00}\right\rangle$ and $\left|\beta_{10}\right\rangle$ we have

$$
\frac{1}{\sqrt{2}}\left(\left|0^{A} 0^{B}\right\rangle \pm\left|1^{A} 1^{B}\right\rangle\right) \rightarrow\left(\mu_{\left|0^{A}\right\rangle}+\mu_{\left|0^{B}\right\rangle}+\mu_{\left|1^{A}\right\rangle}+\mu_{\left|1^{B}\right\rangle}=0\right)
$$

while that for $\left|\beta_{01}\right\rangle$ and $\left|\beta_{11}\right\rangle$ yields

$$
\frac{1}{\sqrt{2}}\left(\left|0^{A} 1^{B}\right\rangle \pm\left|1^{A} 0^{B}\right\rangle\right) \rightarrow\left(\mu_{\left|0^{A}\right\rangle}+\mu_{\left|1^{B}\right\rangle}+\mu_{\left|1^{A}\right\rangle}+\mu_{\left|0^{B}\right\rangle}=0\right)
$$

which confirms the conservation of the spin for maximally entangled states. Finally, we must note that the signs in the middle of the simple bases $\left|0^{A} 0^{B}\right\rangle$ and $\left|1^{A} 1^{B}\right\rangle$ of equation (2.12) do not matter, nor do the signs in the middle of the simple bases $\left|0^{A} 1^{B}\right\rangle$ and $\left|1^{A} 0^{B}\right\rangle$ of equation (2.13). 
Next, the concepts necessary for a better understanding of $\S 3$ will be developed. These concepts are: density matrix, entropy and mutual information [1]. The last one is important as a metric to ponder the degree of correlation between two subsystems. In this way, we recognize three levels of correlation between two subsystems: completely independent, classically-correlated and entangled subsystems. Besides, and as we have already said, we definitively opt for the scalar notation over the Dirac's notation based on <bra| and |ket> [51] since the former respects orientation (thanks to the sign of $\mu$ ), magnitude (thanks to the value of $\mu$ ) and is much more practical and flexible from the arithmetic point of view. Although we will refer to both types of notations (Dirac and scalar), deductions about avatars will be exclusively carried out through scalar notation from now on. Besides, since both entropy and mutual information are scalar magnitudes [1,61], the use of scalar notation will be closer to an elegant treatment of the problem posed. Consequently, we are going to begin with density matrix of the subsystems treated individually,

$$
\rho^{A}=\rho^{B}=\frac{1}{2}(|0\rangle\langle 0|+| 1\rangle\langle 1|)=\frac{1}{2} I=\frac{1}{2}\left[\begin{array}{ll}
1 & 0 \\
0 & 1
\end{array}\right],
$$

where the scalar version of this operator, but based on the above defined $\mu$, will be

$$
r^{A}=r^{B}=\frac{1}{2}\left(\frac{\mu_{|0\rangle}^{2}+\mu_{|1\rangle}^{2}}{\eta}\right)=\frac{1}{2}\left(\frac{\mu_{|0\rangle}^{2}+\mu_{|1\rangle}^{2}}{2}\right),
$$

with $\eta=2$ is an adjustment parameter necessary to compensate the misadjustments existing between the traditional and scalar versions of density matrix. We must note that $r^{A}=r^{B}=1 / 2$, which is consequent with the matrix nature of equation (2.14), in particular, in the context of the entropy that we will see later. However, with respect to the case of the system composed of two subsystems, its density matrix will depend on whether or not these subsystems are entangled. Therefore, for the nonentangled case:

$\rho^{A \cup B}=\rho^{A} \otimes \rho^{B}$,

but for the entangled case, we will have,

$\rho^{A \cup B} \neq \rho^{A} \otimes \rho^{B}$

Although this operator will depend on each of the 3 cases that will be analyzed in $\S 3$. Now, with respect to the entropy, we begin with the traditional definition [1], that is,

$$
S^{A}=S^{B}=-\operatorname{tr}\left[\rho^{A} \log \left(\rho^{A}\right)\right]=-\operatorname{tr}\left[\rho^{B} \log \left(\rho^{B}\right)\right],
$$

then, replacing equation (2.14) into equation (2.18), yields,

$$
S^{A}=S^{B}=-\operatorname{tr}\left[\frac{1}{2}\left[\begin{array}{ll}
1 & 0 \\
0 & 1
\end{array}\right] \log \left(\frac{1}{2}\left[\begin{array}{ll}
1 & 0 \\
0 & 1
\end{array}\right]\right)\right]=1,
$$

while the scalar version of the entropy is,

$$
S^{A}=S^{B}=-\kappa\left[r^{A} \log \left(r^{A}\right)\right]=-\kappa\left[r^{B} \log \left(r^{B}\right)\right],
$$

where, $\kappa$ is another adjustment parameter. Therefore, replacing equation (2.15) into equation (2.20), 
and considering: $\mu_{|0\rangle}=-\mu_{|1\rangle}=1$, we will have a $\kappa=2$, which yields,

$$
S^{A}=S^{B}=-2[1 / 2 \log (1 / 2)]=1 .
$$

In the same way, for a composed system, the entropy is,

$$
S^{A \cup B}=-\operatorname{tr}\left[\rho^{A \cup B} \log \left(\rho^{A \cup B}\right)\right]=-\kappa\left[r^{A \cup B} \log \left(r^{A \cup B}\right)\right] .
$$

In this case, $\kappa$ depends on the degree of correlation (completely independent, classically-correlated, and entangled subsystems) between both subsystems.

Moreover, both in the classical and in the quantum world, the correlations between the subsystems are those established by the additional information. In the case of composite quantum systems, the mutual information $S^{A \cap B}$ is introduced to quantify that additional information, allowing us to obtain the degree of correlation between both subsystems [1],

$$
S^{A \cap B}=S^{A}+S^{B}-S^{A \cup B} \geq 0 .
$$

Therefore, the entropy of the composite system $S^{A \cap B}$ indicates that the uncertainty of a state $\rho^{A \cup B}$ is less than the two subsystems $S^{A}$ and $S^{B}$ added together. In other words, $S^{A \cap B}$ tells us how much more information the composite system can store compared to the individual subsystems that composes it. Besides, $S^{A \cap B}$ measures the distance between the state $\rho^{A \cup B}$ and the non-entangled state $\rho^{A} \otimes \rho^{B}[1]$.

The entropy of the subsystems $S^{A}$ and $S^{B}$ is equal to one in all cases (i.e., $S^{A}=S^{B}=1$ ); while the entropy of the composite system $S^{A \cup B}$ will have different values for each and every of the three cases. For the sake of simplicity, the subsystems are expressed in qubits. Making use of equation (2.23), the main idea is to relate correlations and entanglement to the entropy, and in particular, to the mutual information $S^{A \cap B}$. Then, in $\S 3$, we will present the mutual information of the three types of subsystems: completely independent, classically correlated, and entangled, where the last one is critical for the development of avatars.

\section{The avatars appear on the scene}

This section is devoted to evaluate the degree of correlation between two subsystems based on an important metric called mutual information, which was defined in equation (2.23) and that will be considered for the three mentioned cases above: completely independent, classically correlated and entangled [1]. In these three cases, some byproducts resulting from each type of interaction between such subsystems will appear and we will call them avatars. Unlike the considered original particles, which usually are quite separated from each other, the avatars are always grouped in a single intermediate point in the equidistant geometric center from the original particles and acting as the representatives of these, forming original-avatar pairs. In fact, no matter how much the original particles are separated, the avatars will always be in the middle of them and will have the same value, which will depend exclusively on the original spins involved. In other words, the avatars are always insensitive to the distance between the original particles. So far, we have considered that the original particles are always equals in the three cases, at the most with different polarizations but always equal. However, we will take into account other three conceptually important cases: completely independent particles of different spins, quasi-entanglement between particles of different spins, and a teratological case predicted by this analysis. Generalizations and applications due to the analysis based on avatars but for the exclusive case of entanglement will be addressed in subsequent sections. 
Two more things to take into account before starting with the analysis of the three types of subsystems correlation:

- We are going to define a color code in order to facilitate the interpretation of the different figures involved in this section. For all the cases mentioned here, and as we have seen in $\S 2$, we will continue to associate the belonging of each particle with a superscript: A in the case of Alice and B in the case of Bob. However, given the increasing complexity of the figures as we move forward in this paper, it is more thoughtful and convenient to reinforce this idea by assigning colors to the different spins in each case, therefore, we will use red for Alice and blue for Bob.

- In all cases, the avatars will represent (among many other things) a kind of radiography that will allow us to observe the interior of a process, which inhibited all the individualities of the elements that composed it. The most conspicuous and useful case of all will take place for entangled particles. In this case, the avatars are as physically inaccessible as the original particles they represent; however, this representation is purely operative, that is, the avatars tell us how the original particles behave in reality. The avatars are an individual manifestation of each constituent particle of a collective phenomenon called entanglement, e.g. we know that during entanglement an integrating and unique collective wave-function is associated with any of the bases of the equation (2.11), losing all glimmer of individualism from the constituent original particles or spins. Although the avatars rescue each original constituent element and express it individually and unequivocally (forming indivisible original-avatar pairs), they also act collectively allowing us to better understand the attributes of entanglement, fundamentally in quantum communications [62-67]. Summing up, the avatars that result from an entanglement show us the internal elements within a collective phenomenon in an individual form and how those elements really behave in that context.

\subsection{Completely independent subsystems}

In this case, both subsystems have absolute and complete independence between them, i.e., $\rho^{A \cup B}$ is a Kronecker's product of density matrices like equation (2.16). We can clearly see this independence in Figure 3.1. Therefore, there are no correlations between such subsystems.

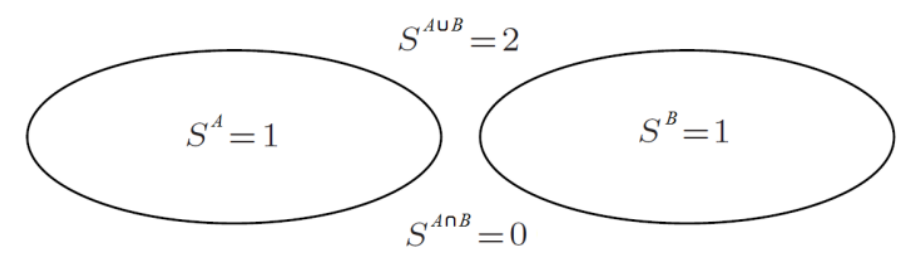

Figure 3.1 Completely independent subsystems.

In the computational basis of $\mathrm{H}^{A} \otimes \mathrm{H}^{B}, \rho^{A \cup B}$ takes the following form,

$$
\rho^{A \cup B}=\frac{1}{4}\left(\left|0^{A}, 0^{B}\right\rangle\left\langle 0^{A}, 0^{B}|+| 0^{A}, 1^{B}\right\rangle\left\langle 0^{A}, 1^{B}|+| 1^{A}, 0^{B}\right\rangle\left\langle 1^{A}, 0^{B}|+| 1^{A}, 1^{B}\right\rangle\left\langle 1^{A}, 1^{B}\right|\right)=\frac{1}{4} I,
$$

where $I$ is a $4 \times 4$ identity matrix. Then, replacing equation (3.1) into equation (2.22), $S^{A \cup B}$ will be

$$
S^{A \cup B}=-\operatorname{tr}\left\{\frac{1}{4}\left[\begin{array}{llll}
1 & 0 & 0 & 0 \\
0 & 1 & 0 & 0 \\
0 & 0 & 1 & 0 \\
0 & 0 & 0 & 1
\end{array}\right] \log \left(\frac{1}{4}\left[\begin{array}{llll}
1 & 0 & 0 & 0 \\
0 & 1 & 0 & 0 \\
0 & 0 & 1 & 0 \\
0 & 0 & 0 & 1
\end{array}\right]\right)\right\}=2 .
$$

Now, introducing the results of equation (2.21) and (3.2) into equation (2.23), 


$$
S^{A \cap B}=S^{A}+S^{B}-S^{A \cup B}=1+1-2=0 .
$$

The result of the equation (3.3) endorses the complete independence of the subsystems. In fact, if we remember the equivalences of $\S 2$ where $|0\rangle \equiv \mu_{|0\rangle},|1\rangle \equiv \mu_{|1\rangle}\left|0^{A} 0^{B}\right\rangle \equiv \mu_{\left|0^{A} 0^{B}\right\rangle}$, and $\left|1^{A} 1^{B}\right\rangle \equiv \mu_{\left|1^{A} 1^{B}\right\rangle}$, then, the scalar version of equation (3.1) will be,

$$
\begin{aligned}
& r^{A \cup B}=\frac{1}{4}\left(\frac{\mu_{\left|0^{A} 0^{B}\right\rangle} \mu_{\left|0^{A} 0^{B}\right\rangle}+\mu_{\left|0^{A} 1^{B}\right\rangle} \mu_{\left|0^{A} 1^{B}\right\rangle}+\mu_{\left|1^{A} 0^{B}\right\rangle} \mu_{\left|1^{A} 0^{B}\right\rangle}+\mu_{\left|1^{A} 1^{B}\right\rangle} \mu_{\left|1^{A^{A} 1^{B}}\right\rangle}}{\eta}\right) \\
& =\frac{1}{4}\left(\frac{\mu_{\left|0^{A, B}\right\rangle}^{2} \mu_{\left|0^{A, B}\right\rangle}^{2}+\left(\mu_{\left|0^{A}\right\rangle} \mu_{\left|1^{B}\right\rangle}\right)\left(\mu_{\left|0^{A}\right\rangle} \mu_{\left|1^{B}\right\rangle}\right)+\left(\mu_{\left|1^{A}\right\rangle} \mu_{\left|0^{B}\right\rangle}\right)\left(\mu_{\left|1^{A}\right\rangle} \mu_{\left|0^{B}\right\rangle}\right)+\left(-\mu_{\left|1^{A, B}\right\rangle}^{2}\right)\left(-\mu_{\left|1^{A, B}\right\rangle}^{2}\right)}{4}\right) \\
& =\frac{1}{4}\left(\frac{\mu_{\left|0^{A, B}\right\rangle}^{4}+\mu_{\left|0^{A}\right\rangle}^{2} \mu_{\left|1^{B}\right\rangle}^{2}+\mu_{\left|1^{A}\right\rangle}^{2} \mu_{\left|0^{B}\right\rangle}^{2}+\mu_{\left|1^{A, B}\right\rangle}^{4}}{\eta}\right)=\left(\frac{\mu_{\left|0^{A, B}\right\rangle}^{4}+2 \mu_{\left|0^{A, B}\right\rangle}^{2} \mu_{\left|1^{A, B}\right\rangle}^{2}+\mu_{\left.||^{A, B}\right\rangle}^{4}}{4^{2}}\right)=\left(\frac{\mu_{\left|0^{A, B}\right\rangle}^{2}+\mu_{\left|1^{A, B}\right\rangle}^{2}}{4}\right)^{2}
\end{aligned}
$$

where $\eta$ will be equal to 4 for the purpose of adjusting its subsequent use into entropy. Besides, we must make the following deduction considering that: $\mu_{\left|0^{A, B}\right\rangle}^{2}=\mu_{\left|1^{A, B}\right\rangle}^{2}=1$. Then, replacing equation (3.4) into the scalar version of equation (2.22) with $\kappa=4$, and $S^{A \cup B}=2$ from equation (3.2), we will have,

$$
S^{A \cup B}=2=-\kappa\left[r^{A \cup B} \log \left(r^{A \cup B}\right)\right]=-4\left[\left(\frac{\mu_{\left.\left.\right|^{\wedge, B}\right\rangle}^{2}+\mu_{\left|1^{\wedge, B}\right\rangle}^{2}}{4}\right)^{2} \log \left(\left(\frac{\mu_{\left.0^{1, B}\right\rangle}^{2}+\mu_{\left.\left.\right|^{1, B}\right\rangle}^{2}}{4}\right)^{2}\right)\right]
$$

Replacing $\mu_{\left|0^{A, B}\right\rangle}^{2}=\mu_{\left|1^{A, B}\right\rangle}^{2}=1$ outside logarithm, and making additions and subtractions that do not alter equation (3.5),

$$
\begin{aligned}
& 1=-\log \left(\frac{\mu_{\left.0^{A, B}\right\rangle}^{2}+\mu_{\left.\left.\right|^{1, B}\right\rangle}^{2}}{4}\right)+\log \left(\frac{\mu_{\left.0^{\alpha, B}\right\rangle}^{2}}{2}\right)-\log \left(\frac{\mu_{\left.\left.\right|^{\alpha, B}\right\rangle}^{2}}{2}\right)+\log \left(\frac{\mu_{\left.\left.\right|^{1, B}\right\rangle}^{2}}{2}\right)-\log \left(\frac{\mu_{\left.1^{A, B}\right\rangle}^{2}}{2}\right) \\
& =-\log \left(\frac{\left.\mu_{\left.0^{A, B}\right\rangle}^{2}+\mu_{\left.\left.\right|^{1, B}\right\rangle}^{2}\right\rangle}{4}\right)+\log \left(\frac{\mu_{\left.0^{A, B}\right\rangle}^{2}}{2}\right)+1+\log \left(\frac{\mu_{\left.\left.\right|^{1, B}\right\rangle}^{2}}{2}\right)+1
\end{aligned}
$$

and,

$$
\begin{aligned}
& -1=-\log \left(\frac{\mu_{\left.0^{\Lambda, B}\right\rangle}^{2}+\mu_{\left.\left.\right|^{1, B}\right\rangle}^{2}}{4}\right)+\log \left(\frac{\mu_{\left|0^{\Lambda, B}\right\rangle}^{2}}{2}\right)+\log \left(\frac{\mu_{\left|1^{\Lambda, B}\right\rangle}^{2}}{2}\right)
\end{aligned}
$$

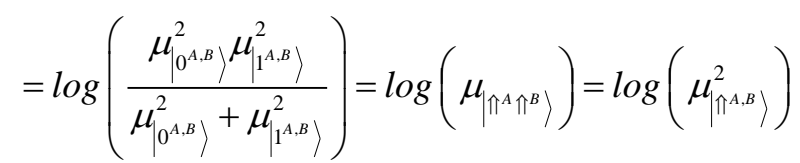

Finally, 


$$
\begin{aligned}
\mu_{\left|\Uparrow^{A} \Uparrow^{B}\right\rangle}= & \mu_{\left|\Uparrow^{A, B}\right\rangle}^{2}=\frac{\mu_{\left|0^{A, B}\right\rangle}^{2} \mu_{\left|1^{A, B}\right\rangle}^{2}}{\mu_{\left|0^{A, B}\right\rangle}^{2}+\mu_{\left|1^{A, B}\right\rangle}^{2}}=\mu_{\left|0^{A, B}\right\rangle}^{2} / / \mu_{\left|1^{A, B}\right\rangle}^{2} \\
= & \frac{\mu_{\left|0^{A} 0^{B}\right\rangle}\left(-\mu_{\left|1^{A} 1^{B}\right\rangle}\right)}{\mu_{\left|0^{A} 0^{B}\right\rangle}+\left(-\mu_{\left|1^{A} 1^{B}\right\rangle}\right)}=\mu_{\left|0^{A} 0^{B}\right\rangle} / /\left(-\mu_{\left|1^{A} 1^{B}\right\rangle}\right)=2^{-1}=\frac{1}{2}
\end{aligned}
$$

where "//"" is the parallel operator, $\left|\Uparrow^{A} \Uparrow^{B}\right\rangle$ is the resulting equivalent spin for this level of correlation, being $\left|\Uparrow^{A} \Uparrow^{B}\right\rangle \equiv \mu_{\left|\Uparrow^{A} \Uparrow^{B}\right\rangle}$, and $\mu_{\left|\Uparrow^{A} \Uparrow^{B}\right\rangle}=\mu_{\left|\Uparrow^{A, B}\right\rangle}^{2}$ is the second-degree-equivalent spin for completely independent subsystems with a value of $1 / 2$. It is evident that it would have been impossible to deduce $\mu_{\left|\Uparrow^{A} \Uparrow^{B}\right\rangle}=\mu_{\left|\Uparrow^{A, B}\right\rangle}^{2}$ with the Dirac's notation based on <bra| and |ket> [51]. The graphic interpretation of equation (3.8) can be seen in Figure 3.2.

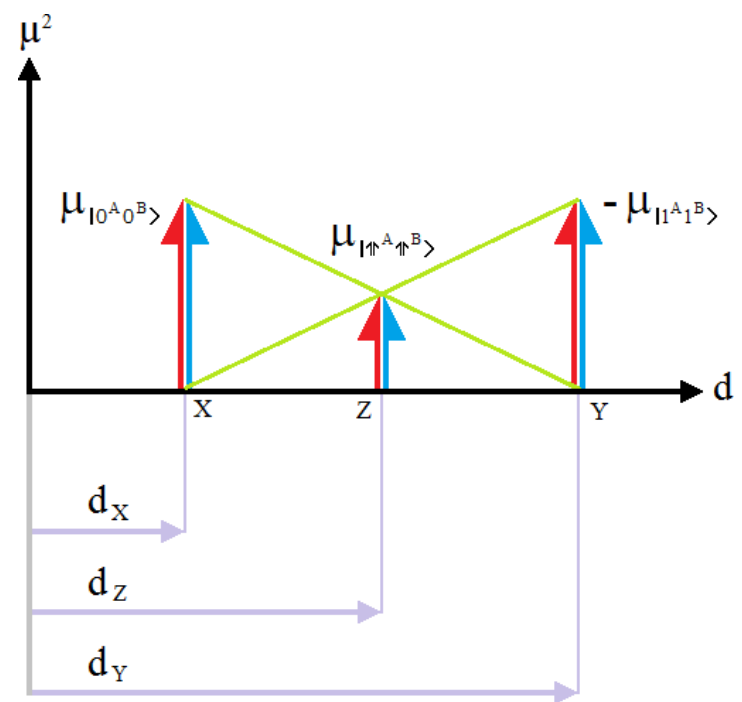

Figure 3.2 Second-degree-equivalent spin $\left(\mu^{2}\right)$ and distances for completely independent subsystems.

Figure 3.2 shows that $\mu_{\left|0^{A} 0^{B}\right\rangle}$ is in position $X$, and $-\mu_{\left|1^{A} 1^{B}\right\rangle}$ is in position $Y$, where, the intersection of both light green lines allows us to determine the value (according to equation 3.8) and position $\mathrm{Z}$ (according to equation 3.9) of $\mu_{\left|\Uparrow^{A} \Uparrow^{B}\right\rangle}=\mu_{\left|\Uparrow^{A, B}\right\rangle}^{2}$,

$d_{Z}=\frac{\mu_{\left|0^{A} 0^{B}\right\rangle} d_{Y}+\left(-\mu_{\left|1^{A} 1^{B}\right\rangle}\right) d_{X}}{\mu_{\left|0^{A} 0^{B}\right\rangle}+\left(-\mu_{\left|1^{A} 1^{B}\right\rangle}\right)}$

where $d_{Z}=d_{\left|\Uparrow^{A} \Uparrow^{B}\right\rangle}, d_{X}=d_{\left|0^{A} 0^{B}\right\rangle}$, and $d_{Y}=d_{\left|1^{A} 1^{B}\right\rangle}$. For example, in Figure 3.2, if $d_{X}=2.8$ meters and $d_{Y}=9.8$ meters, then $d_{Z}=6.3$ meters, which matches the proportions of the graph. Besides, as we have said, $\mu_{\left|\Uparrow^{A} \Uparrow^{B}\right\rangle}=\mu_{\left|\Uparrow^{A, B}\right\rangle}^{2}$ arises from the intersection of the two light green lines, one connects the upper 
part of $\mu_{\left|0^{A} 0^{B}\right\rangle}$ with the base of $-\mu_{\left|1^{A} 1^{B}\right\rangle}$ and the other connects the base $\mu_{\left|0^{A} 0^{B}\right\rangle}$ with the upper part of $-\mu_{\left|1^{A} 1^{B}\right\rangle}$. In short, this is - in itself - the geometric interpretation of the parallel operator.

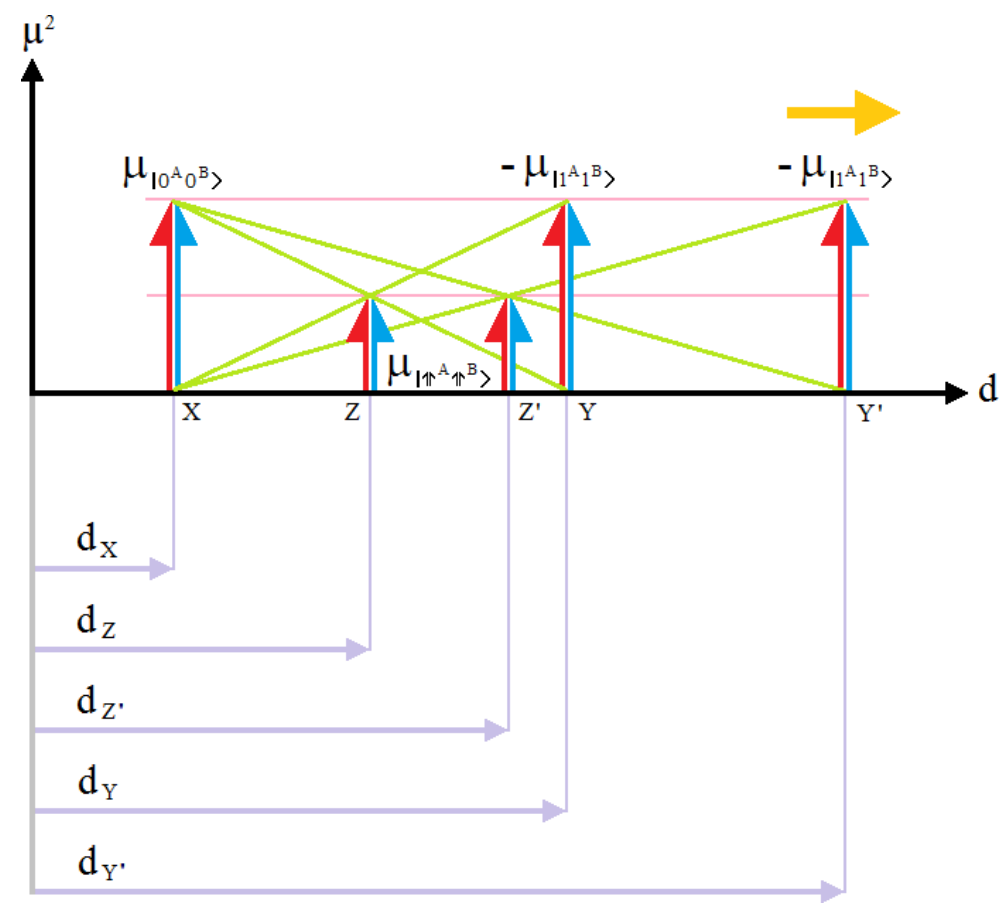

Figure 3.3 Completely independent subsystems with an increase in the separation of the original spins.

Now, based on Figure 3.3, if we move $-\mu_{\left|1^{A} 1^{B}\right\rangle}$ to position $Y^{\prime}$, and we leave $\mu_{\left|0^{A} 0^{B}\right\rangle}$ in its original position $X$, and reapply the parallel operator of equation (3.8) to this new configuration, we will see that a new $\mu_{\left|\Uparrow^{A} \Uparrow^{B}\right\rangle}=\mu_{\left|\Uparrow^{A, B}\right\rangle}^{2}$ appears as a result of the two new intersected light green lines, but now in position Z', which has a value of $1 / 2$ again, i.e., similar to the previous case of Figure 3.2. We can see this new result in Figure 3.3, where, the pink lines are parallel to the thick black line of the base and perpendicular to the arrows representing the spins. In other words, the pink lines indicate that the heights of the arrows that represent the spins $\mu_{\left|\Uparrow^{A} \pi^{B}\right\rangle}$ are preserved regardless of the distance between both original particles $\left(\mu_{\left|0^{A} 0^{B}\right\rangle},-\mu_{\left|1^{A} 1^{B}\right\rangle}\right)$ on which the parallel operator is applied. This indicates that the parallel operator is insensitive to the distance between the two original spins. This first observation is absolutely consistent with the behavior of the entanglement which is permanently registered in the laboratories [31, 49]. Thus, and considering equation (3.10)

$d_{Z^{\prime}}=\frac{\mu_{\left|0^{A} 0^{B}\right\rangle^{B}} d_{Y^{\prime}}+\left(-\mu_{\left|1^{A^{A} B}\right\rangle}\right) d_{X}}{\mu_{\left|0^{A} 0^{B}\right\rangle}+\left(-\mu_{\left|1^{A} 1^{B}\right\rangle}\right)}$

it is evident that if $d_{Y^{\prime}}>d_{Y}$ then $d_{Z^{\prime}}>d_{Z}$, although the second-degree-equivalent spin will be identical in both cases. Now, if we apply square root to both sides of equation (3.8), we will obtain the equivalent of the first-degree-equivalent spins or avatars, 


$$
\begin{aligned}
{\left[\mu_{\left|\Uparrow^{A, B}\right\rangle}, \mu_{\left|\Downarrow^{A, B}\right\rangle}\right] } & = \pm \sqrt{\mu_{\left|\Uparrow^{A} \Uparrow^{B}\right\rangle}}= \pm \sqrt{\mu_{\left|\Uparrow^{A, B}\right\rangle}^{2}}= \pm \sqrt{\frac{\mu_{\left|0^{A, B}\right\rangle}^{2} \mu_{\left.1^{A, B}\right\rangle}^{2}}{\left.\mu_{\left|0^{A, B}\right\rangle}^{2}+\mu_{\left|1^{A, B}\right\rangle}^{2}\right\rangle}} \\
& =\frac{ \pm \mu_{\left|0^{A, B}\right\rangle}}{\sqrt{1+\left(\frac{\left.\mu_{\left|0^{A, B}\right\rangle}\right\rangle}{\mu_{\left|1^{A, B}\right\rangle}}\right)^{2}}}= \pm \mu_{\left|0^{A, B}\right\rangle} \zeta= \pm \frac{\sqrt{2}}{2}
\end{aligned}
$$

where

$$
\zeta=\frac{1}{\sqrt{1+\left(\frac{\mu_{\left|0^{A, B}\right\rangle}}{\mu_{\left|1^{A, B}\right\rangle}}\right)^{2}}}
$$

it is an homothetic scale factor [68-70] which can be interpreted in Figure 3.4.

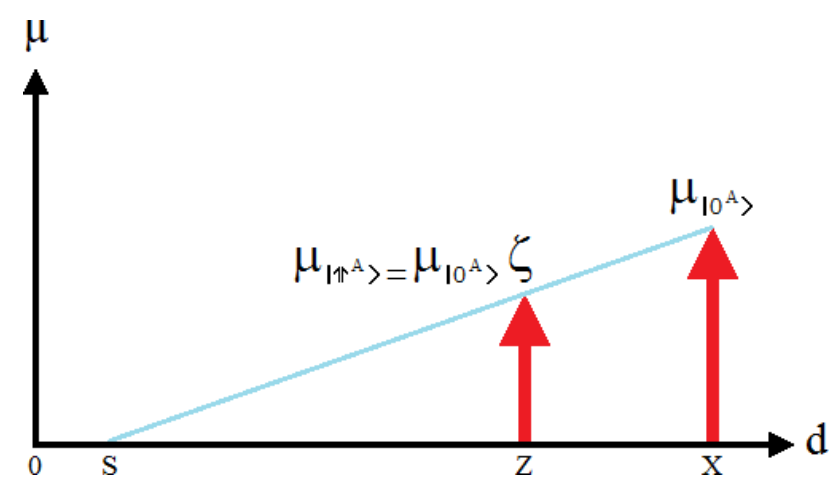

Figure 3.4 Homothety between an original spin and its own avatar.

In such figure, point $S$ is the homothetic center, and each segment is contracted in a $\zeta$ factor inasmuch as $\zeta=\sqrt{2} / 2<1$. For example,

$$
Z \rightarrow S+\zeta \overrightarrow{S X}
$$

which is absolutely consequent with the contraction that suffers the avatars respect to the originals

$$
\begin{aligned}
& \mu_{\left|\Uparrow^{A, B}\right\rangle}=\mu_{\left|0^{A, B}\right\rangle} \zeta \\
& \mu_{\left|\Downarrow^{A, B}\right\rangle}=\mu_{\left|1^{A, B}\right\rangle} \zeta
\end{aligned}
$$

The notion of homothetic transformation [68] is going to be fundamental to development the rest of this paper. Moreover, the upper part of Figure 3.5 shows the second-degree-equivalent spin $\left(\mu^{2}\right)$, while the lower part of the same figure shows the first-degree-equivalent spins $(\mu)$ or avatars. The position of the avatars is determined by the intersection of the grey line (which goes from the tip of $\mu_{\left|0^{A, B}\right\rangle}$ to the tip of $\left.\mu_{\left|1^{A, B}\right\rangle}\right)$ with the thick black line or distance axis $(d)$. Formally, 


$$
d_{Z}=\frac{\sqrt{\mu_{\left|0^{A} 0^{B}\right\rangle}} d_{Y}+\sqrt{-\mu_{\left|1^{A} 1^{B}\right\rangle}} d_{X}}{\sqrt{\mu_{\left|0^{A} 0^{B}\right\rangle}}+\sqrt{-\mu_{\left|1^{A} 1^{B}\right\rangle}}}=\frac{\mu_{\left|0^{A, B}\right\rangle} d_{Y}+\left(-\mu_{\left|1^{A, B}\right\rangle}\right) d_{X}}{\mu_{\left|0^{A, B}\right\rangle}+\left(-\mu_{\left|1^{A, B}\right\rangle}\right)}
$$

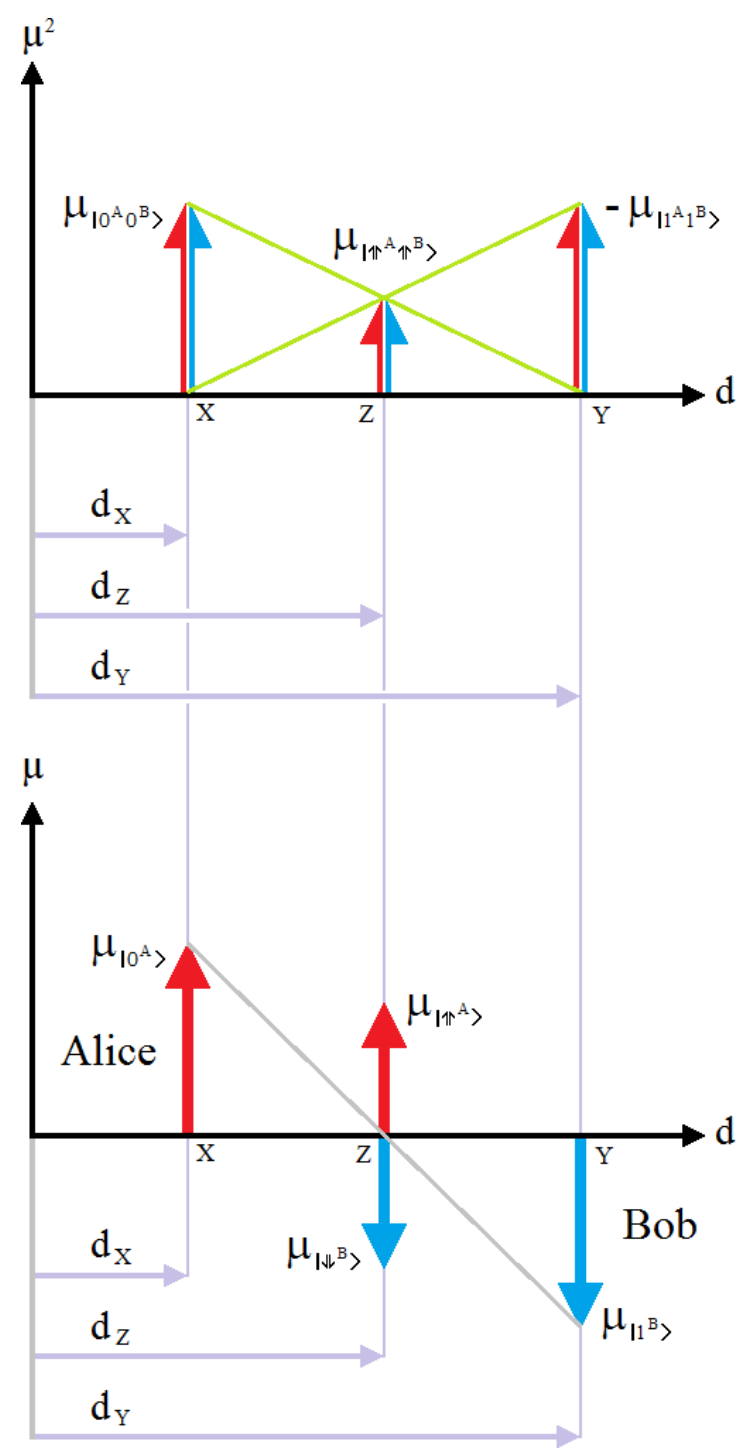

Figure 3.5 Second-degree-equivalent spin $\left(\mu^{2}\right)$ and first-degree-equivalent spins $(\mu)$ or avatars, including distances for completely independent subsystems.

The lower part of Figure 3.5 shows us the original spins in an identical configuration to the lower part of Figure 2.1, which represents the spins that are original members of an entanglement but that after a quantum measurement (and as a consequence of the loss of entanglement) are completely independent. On the other hand, the avatars only represent those original spins at an operative intermediate point.

When the original particles have the same spin, both equations (3.9) and (3.15) match, i.e., second and first-degree-equivalent spins have the same allocation. In fact, we can represent both equations like a simple average

$d_{Z}=\frac{d_{Y}+d_{X}}{2}$. 
But, what happens with the equivalent spins and their locations when the original spins have different values? This case is represented in Figure 3.6.

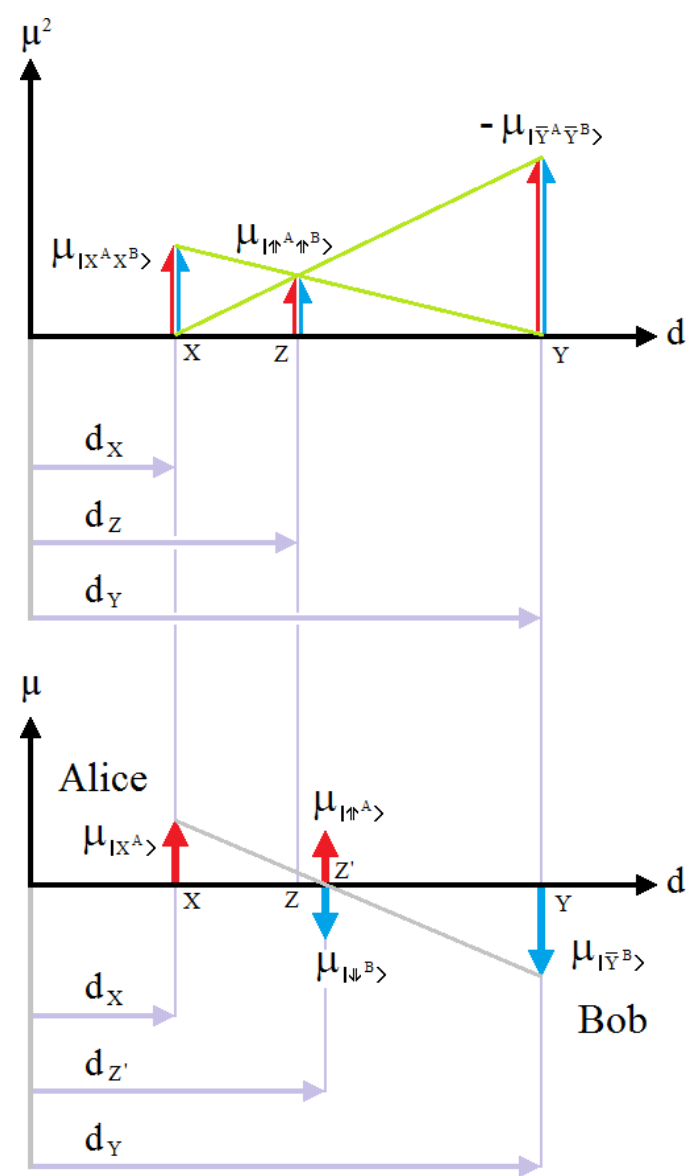

Figure 3.6 Second-degree-equivalent spin $\left(\mu^{2}\right)$ and first-degree-equivalent $(\mu)$ or avatars, including distances for completely independent subsystems but for particles of different spins.

In the upper part of Figure 3.6, the intersections of both light green lines give us the value and allocation of the second-degree-equivalent spin $\left(\mu^{2}\right)$. Its value responds to equation (3.8), surely, with a different result than $1 / 2$, while its allocation responds to equation (3.9), but, replacing $\left\{\mu_{\left|0^{A} 0^{B}\right\rangle},-\mu_{\left.\left.\right|^{A^{A} B^{B}}\right\rangle}\right\}$ with $\left\{\mu_{\left|X^{A} X^{B}\right\rangle},-\mu_{\left.\bar{Y}^{A} \bar{Y}^{B}\right\rangle}\right\}$. The first thing we noticed from Figure 3.6 is that since the original spins are not equal, then, the avatars are not at the midpoint equidistant between those originals. Now, what happens to the equivalent of the first-degree-equivalent spins or avatars? If for this case we apply the equation (3.15), also replacing $\left\{\mu_{\left|0^{A} 0^{B}\right\rangle},-\mu_{\left|1^{A} 1^{B}\right\rangle}\right\}$ with $\left\{\mu_{\left|X^{A} X^{B}\right\rangle},-\mu_{\left.\bar{Y}^{A} \bar{Y}^{B}\right\rangle}\right\}$, respectively, the lower part of Figure 3.6 shows us that the allocation of the second-degree-equivalent spin does not match with the allocation of first-degree-equivalent spins or avatars. The allocation can be tested graphically thanks to the intersection of the grey line (which goes from the tip of $\mu_{\left|X^{A, B}\right\rangle}$ to the tip of $\mu_{\left|\bar{Y}^{A, B}\right\rangle}$ ) with thick black line or distance axis $(d)$. In other words, points $Z$ and $Z$ do not coincide although the avatars (also in this case) remain together. Finally, the particles of the second degree do not preserve the spin, i.e., $\mu_{\left|X^{A} X^{B}\right\rangle}+\mu_{\left|\bar{Y}^{A} \bar{Y}^{B}\right\rangle} \neq 0$, although the avatars always do it: $\mu_{\left|\Uparrow^{A}\right\rangle}+\mu_{\left|\|^{B}\right\rangle}=0$. 
In completely independent particles, like the ones treated here, with equal or different spins, the avatars represent no more than an intermediate presence or projection at an intermediate point. It is merely representative in the same way as it is with the equivalent mass from the independent masses of a pair of nuclei in some diatomic molecules [5]. In this case, the equivalent mass is also calculated through the parallel operator. This equivalent mass is also known as reduced mass and it is clear that the masses of both nuclei do not have any kind of correlation between them.

Consequently, given the pairs of the original-avatar type that have been formed, and taking into account that $\left|\Uparrow^{A, B}\right\rangle \equiv \mu_{\left|\Uparrow^{A, B}\right\rangle},\left|\Downarrow^{A, B}\right\rangle \equiv \mu_{\left|\Downarrow^{A, B}\right\rangle},\left|0^{A, B}\right\rangle \equiv \mu_{\left|0^{A, B}\right\rangle},\left|1^{A, B}\right\rangle \equiv \mu_{\left|1^{A, B}\right\rangle}$, an interesting question arises: is there a unitary operator that takes us from the original spin to the avatar? For example, if we represent such transition for Figure 3.5 with an equation,

$$
\begin{aligned}
& \left|\Uparrow^{A, B}\right\rangle=U\left|0^{A, B}\right\rangle \\
& \left|\Downarrow^{A, B}\right\rangle=U\left|1^{A, B}\right\rangle
\end{aligned}
$$

we automatically ask ourselves if the equation (3.17) is equivalent to equation (3.14) and if the operator $U$ is really unitary, since if it is not, then, the avatars are not pure states, i.e., they would not be found on the Bloch's sphere [51-53], which would be a direct consequence of Homothetic Transformation [68-70]. Besides, does the operator $U$ have an inverse? Well, if it does not, then the avatars would be involved in a non-quantum process or would be fully responsible for it.

\subsection{Classically-correlated subsystems}

In this case, see Figure 3.7, it is possible to create correlations without obtaining entanglement for $S^{A \cap B} \neq 0$. For example, let us do this with the separable mixture of pure product states,

$\rho^{A \cup B}=\frac{1}{2}\left(\left|0^{A}, 0^{B}\right\rangle\left\langle 0^{A}, 0^{B}|+| 1^{A}, 1^{B}\right\rangle\left\langle 1^{A}, 1^{B}\right|\right)$

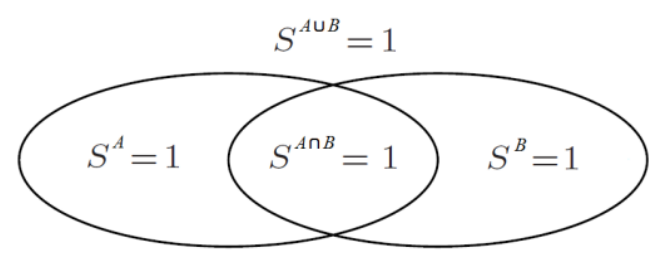

Figure 3.7 Classically-correlated subsystems.

As we can see, $\rho^{A \cup B}$ of equation (3.18) contains fewer terms than the completely independent case of equation (3.1). In the computational basis of $\mathrm{H}^{A} \otimes \mathrm{H}^{B}, \rho^{A \cup B}$ will be:

$\rho^{A \cup B}=\frac{1}{2}\left[\begin{array}{llll}1 & 0 & 0 & 0 \\ 0 & 0 & 0 & 0 \\ 0 & 0 & 0 & 0 \\ 0 & 0 & 0 & 1\end{array}\right]$.

Therefore, replacing equation (3.19) into equation (2.22), $S^{A \cup B}$ will be 
$S^{A \cup B}=-\operatorname{tr}\left\{\frac{1}{2}\left[\begin{array}{llll}1 & 0 & 0 & 0 \\ 0 & 0 & 0 & 0 \\ 0 & 0 & 0 & 0 \\ 0 & 0 & 0 & 1\end{array}\right] \log \left(\frac{1}{2}\left[\begin{array}{llll}1 & 0 & 0 & 0 \\ 0 & 0 & 0 & 0 \\ 0 & 0 & 0 & 0 \\ 0 & 0 & 0 & 1\end{array}\right]\right)\right\}=1 \cdot$

Next, introducing the results of equation (2.21) and (3.20) into equation (2.23), yields the following

$S^{A \cap B}=S^{A}+S^{B}-S^{A \cup B}=1+1-1=1$

This result brings out a certain degree of correlation between the subsystems. Then, if we remember the equivalences of equation (2.8), the scalar version of equation (3.18) will be,

$$
\begin{aligned}
& r^{A \cup B}=\frac{1}{2}\left(\frac{\mu_{\left|0^{A} 0^{B}\right\rangle} \mu_{\left|0^{A} 0^{B}\right\rangle}+\mu_{\left|1^{A} 1^{B}\right\rangle}}{\eta} \mu_{\left|1^{A} 1^{B}\right\rangle}\right)=\frac{1}{2}\left(\frac{\mu_{\left|0^{A, B}\right\rangle}^{2} \mu_{\left|0^{A, B}\right\rangle}^{2}+\left(-\mu_{\left|1^{A, B}\right\rangle}^{2}\right)\left(-\mu_{\left|1^{A, B}\right\rangle}^{2}\right)}{4}\right) \\
& =\frac{1}{2}\left(\frac{\mu_{\left|0^{A, B}\right\rangle}^{4}+\mu_{\left|1^{A, B}\right\rangle}^{4}}{4}\right)=\frac{\mu_{\left|0^{A, B}\right\rangle}^{4}+\mu_{\left|1^{A, B}\right\rangle}^{4}}{8}
\end{aligned}
$$

where $\eta=4$ for the same reasons as for the completely independent case, where we also consider: $\mu_{\left|0^{A, B}\right\rangle}^{4}=\mu_{\left|1^{A, B}\right\rangle}^{4}=1$. Then, replacing equation (3.22) into the scalar version of equation (2.22) with $\kappa=$ 2, and $S^{A \cup B}=1$ from equation (3.20), we will have,

$$
S^{A \cup B}=1=-\kappa\left[r^{A \cup B} \log \left(r^{A \cup B}\right)\right]=-2\left[\left(\frac{\mu_{\left|0^{A, B}\right\rangle}^{4}+\mu_{\left|1^{A, B}\right\rangle}^{4}}{8}\right) \log \left(\left(\frac{\mu_{\left|0^{A, B}\right\rangle}^{4}+\mu_{\left|1^{A, B}\right\rangle}^{4}}{8}\right)\right] .\right.
$$

Replacing $\mu_{\left|0^{A, B}\right\rangle}^{4}=\mu_{\left|1^{A, B}\right\rangle}^{4}=1$ outside logarithm,

$2=-\log \left(\frac{\left.\mu_{\left|0^{A, B}\right\rangle}^{4}+\mu_{\left.||^{1, B}\right\rangle}^{4}\right\rangle}{8}\right)=-\log \left(\mu_{\left|0^{A, B}\right\rangle}^{4}+\mu_{\left|1^{A, B}\right\rangle}^{4}\right)+3$

Simplifying and making additions and subtractions that do not alter equation (3.24),

$$
-1=-\log \left(\mu_{\left|0^{A, B}\right\rangle}^{4}+\mu_{\left|1^{A, B}\right\rangle}^{4}\right)+\log \left(\mu_{\left|0^{A, B}\right\rangle}^{4}\right)-\log \left(\mu_{\left|0^{A, B}\right\rangle}^{4}\right)+\log \left(\mu_{\left|1^{A, B}\right\rangle}^{4}\right)-\log \left(\mu_{\left|1^{A, B}\right\rangle}^{4}\right),
$$

being $-\log \left(\mu_{\left|0^{A, B}\right\rangle}^{4}\right)=-\log \left(\mu_{\left.\left.\right|^{A, B}\right\rangle}^{4}\right)=0$, then,

$$
-1=\log \left(\frac{\mu_{\left|0^{A, B}\right\rangle}^{4} \mu_{\left|1^{A, B}\right\rangle}^{4}}{\mu_{\left|0^{A, B}\right\rangle}^{4}+\mu_{\left|1^{A, B}\right\rangle}^{4}}\right)=\log \left(\mu_{\left|\Uparrow^{A} \Uparrow^{B}\right\rangle}^{2}\right)=\log \left(\mu_{\left|\Uparrow^{A, B}\right\rangle}^{4}\right),
$$

Finally, 


$$
\mu_{\left|\Uparrow^{A} \Uparrow^{B}\right\rangle}^{2}=\mu_{\left|\pi^{A, B}\right\rangle}^{4}=\frac{\left.\mu_{\left|0^{A, B}\right\rangle}^{4} \mu_{\left|1^{A, B}\right\rangle}^{4}\right\rangle}{\mu_{\left|0^{A, B}\right\rangle}^{4}+\mu_{\left|1^{A, B}\right\rangle}^{4}}=\mu_{\left|0^{A, B}\right\rangle}^{4} / / \mu_{\left|1^{A, B}\right\rangle}^{4}=2^{-1}=\frac{1}{2} .
$$

Anew, this would have remained hidden if not for this type of treatment: the scalar notation. Besides, and as in the previous case (completely independent), the first-degree-equivalent spins ( $\mu$ ), or avatars, represent (in some way) the original spins involved in a similar analysis to the one made for Figure 3.3, and with a similar result, because, in this case too, the parallel operator is completely insensitive to the distance between the original spins. Then, if we apply the fourth root to both sides of equation (3.27), we obtain the final values of the avatars.

$$
\begin{aligned}
& {\left[\mu_{\Uparrow^{A, B},}, \mu_{\Rightarrow A, B}, \mu_{\Downarrow A, B}, \mu_{\models^{A, B}}\right]=\sqrt[4]{\mu_{\left|\Uparrow^{A, B}\right\rangle}^{4}}[+1,+i,-1,-i]=\sqrt[4]{\mu_{\left|0^{A, B}\right\rangle}^{4} / / \mu_{\left|1^{A, B}\right\rangle}^{4}}[+1,+i,-1,-i]} \\
& =\sqrt[4]{\frac{\left.\mu_{\left|0^{A, B}\right\rangle}^{4} \mu_{\left|1^{A, B}\right\rangle}^{4}\right\rangle}{\left.\mu_{\left|0^{4, B}\right\rangle}^{4}+\mu_{\left|1^{A, B}\right\rangle}^{4}\right\rangle}}[+1,+i,-1,-i]=\frac{\mu_{\left|0^{A, B}\right\rangle}[+1,+i,-1,-i]}{\sqrt[4]{1+\left(\frac{\mu_{\left|0^{A, B}\right\rangle}}{\mu_{\left|1^{A, B}\right\rangle}}\right)^{4}}} \\
& =\mu_{\left|0^{A, B}\right\rangle} \varsigma[+1,+i,-1,-i]
\end{aligned}
$$

being

$$
\varsigma=\frac{1}{\sqrt[4]{1+\left(\frac{\mu_{\left|0^{A, B}\right\rangle}}{\mu_{\left|1^{A, B}\right\rangle}}\right)^{4}}}
$$

Here too, the avatars suffer a contraction with respect to the original spins in a factor $\varsigma$

$$
\begin{aligned}
& \mu_{\Uparrow^{A, B}}=\mu_{\left|0^{A, B}\right\rangle} \varsigma \\
& \mu_{\Rightarrow^{A, B}}=\mathrm{i} \mu_{\left|0^{A, B}\right\rangle} \varsigma \\
& \mu_{\Downarrow^{A, B}}=-\mu_{\left|0^{A, B}\right\rangle} \varsigma=\mu_{\left|1^{A, B}\right\rangle} \varsigma \\
& \mu_{\models^{A, B}}=-\mathrm{i} \mu_{\left|0^{A, B}\right\rangle} \varsigma=\mathrm{i} \mu_{\left|1^{A, B}\right\rangle} \varsigma
\end{aligned}
$$

while, the avatars' positions can be also calculated with equation (3.15). Moreover, equation (3.30) shows that here there is also a homothety [68] between the original spins and their avatars. Finally, we will not go into detail with this degree of correlation because it does not align with the central idea of this paper.

\subsection{Entangled subsystems}

For this case, we are going to resort to Figure 3.8. First, we will take one of Bell's states of equation (2.11) and as an example, specifically $\left|\beta_{00}\right\rangle=\left|\Phi^{+}\right\rangle$, with $\rho^{A \cup B}$ from equation (3.31),

$$
\rho^{A \cup B}=\frac{1}{4}\left(\left|0^{A}, 0^{B}\right\rangle\left\langle 0^{A}, 0^{B}|+| 0^{A}, 0^{B}\right\rangle\left\langle 1^{A}, 1^{B}|+| 1^{A}, 1^{B}\right\rangle\left\langle 0^{A}, 0^{B}|+| 1^{A}, 1^{B}\right\rangle\left\langle 1^{A}, 1^{B}\right|\right) .
$$




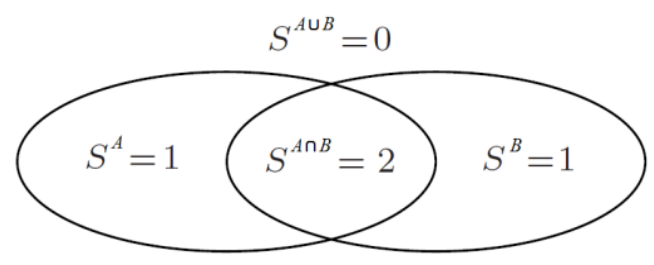

Figure 3.8 Entangled subsystems.

Besides, given that for this case $S^{A \cup B}=0$, we are going to introduce this value and the results of equation (2.19) into equation (2.23). Consequently, we will obtain

$S^{A \cap B}=S^{A}+S^{B}-S^{A \cup B}=1+1-0=2$.

On the other hand, as in the other two cases (completely independent and classically correlated), we will use, the scalar notation where the polarizations or orientations of the spins $\left(\mu\right.$ and $\left.\mu^{2}\right)$ are represented by the sign of a scalar: spin-up $\equiv+1$ and spin-down $\equiv-1$. We will start with the calculation of $r^{A \cup B}$,

$$
\begin{aligned}
& r^{A \cup B}=\frac{1}{4}\left(\frac{\mu_{\left|0^{A} 0^{B}\right\rangle} \mu_{\left|0^{A} 0^{B}\right\rangle}+\mu_{\left|0^{A} 0^{B}\right\rangle} \mu_{\left|1^{A} 1^{B}\right\rangle}+\mu_{\left|1^{A} 1^{B}\right\rangle} \mu_{\left|0^{A} 0^{B}\right\rangle}+\mu_{\left|1^{A} 1^{B}\right\rangle} \mu_{\left|1^{A} 1^{B}\right\rangle}}{\eta}\right) \\
& =4\left(\frac{\mu_{\left|0^{A, B}\right\rangle}^{2} \mu_{\left|0^{A, B}\right\rangle}^{2}+\mu_{\left|0^{A, B}\right\rangle}^{2}\left(-\mu_{\left|1^{A, B}\right\rangle}^{2}\right)+\left(-\mu_{\left|1^{1, B}\right\rangle}^{2}\right) \mu_{\left|0^{A, B}\right\rangle}^{2}+\left(-\mu_{\left.\left.\right|^{1, B}\right\rangle}^{2}\right)\left(-\mu_{\left|1^{1, B}\right\rangle}^{2}\right)}{4}\right), \\
& =\frac{1}{4}\left(\frac{\mu_{\left|0^{A, B}\right\rangle}^{4}-2 \mu_{\left|0^{A, B}\right\rangle}^{2} \mu_{\left|1^{A, B}\right\rangle}^{2}+\mu_{\left.||^{A, B}\right\rangle}^{4}}{4}\right)=\left(\frac{\mu_{\left|0^{A, B}\right\rangle}^{2}-\mu_{\left|1^{A, B}\right\rangle}^{2}}{4}\right)^{2}
\end{aligned}
$$

where we need to use $\eta=4$ for the same reasons that we had in the previous cases, in fact, $\eta$ has always the same value, where we must remember that: $\mu_{\left|0^{A, B}\right\rangle}^{2}=\mu_{\left|1^{A, B}\right\rangle}^{2}=1$. Then, replacing equation (3.33) into the scalar version of equation (2.22) with $\kappa=4$, we will have,

$$
\begin{aligned}
S^{A \cup B} & =0=-\kappa\left[r^{A \cup B} \log \left(r^{A \cup B}\right)\right]=-4\left[\left(\frac{\mu_{\left|0^{A, B}\right\rangle}^{2}-\mu_{\left.\left.\right|^{A, B}\right\rangle}^{2}}{4}\right)^{2} \log \left(\left(\frac{\mu_{\left|0^{A, B}\right\rangle}^{2}-\mu_{\left.\left.\right|^{A, B}\right\rangle}^{2}}{4}\right)^{2}\right)\right] \\
& =-\frac{1}{2}\left[\left(\mu_{\left|0^{A, B}\right\rangle}^{2}-\mu_{\left.\left.\right|^{A, B}\right\rangle}^{2}\right)^{2} \log \left(\frac{\mu_{\left.||^{A, B}\right\rangle}^{2}-\mu_{\left|1^{A, B}\right\rangle}^{2}}{4}\right)\right]
\end{aligned}
$$

Clearing equation (3.34) appropriately,

$$
\frac{0}{\frac{1}{2}\left(\mu_{\left|0^{A, B}\right\rangle}^{2}-\mu_{\left.\left.\right|^{A, B}\right\rangle}^{2}\right)^{2}}=\infty=-\log \left(\frac{\mu_{\left|0^{A, B}\right\rangle}^{2}-\mu_{\left|1^{A, B}\right\rangle}^{2}}{4}\right) .
$$

That is to say, on the left side of equation (3.35), denominator tends to zero with more power than the 
numerator. In fact, the right side verifies it, because, $-\log (0)=\infty$. Now, doing additions and subtractions that do not alter equation (3.35) we will have

$$
\begin{aligned}
\infty & =-\log \left(\frac{\mu_{\left|0^{A, B}\right\rangle}^{2}-\mu_{\left|1^{A, B}\right\rangle}^{2}}{4}\right)+\log \left(\frac{\mu_{\left|0^{A, B}\right\rangle}^{2}}{2}\right)-\log \left(\frac{\mu_{\left|0^{A, B}\right\rangle}^{2}}{2}\right)+\log \left(\frac{\mu_{\left|1^{A, B}\right\rangle}^{2}}{2}\right)-\log \left(\frac{\mu_{\left|1^{A, B}\right\rangle}^{2}}{2}\right) \\
& =-\log \left(\frac{\mu_{\left|0^{A, B}\right\rangle}^{2}-\mu_{\left.||^{A, B}\right\rangle}^{2}}{4}\right)+\log \left(\frac{\mu_{\left|0^{A, B}\right\rangle}^{2}}{2}\right)+1+\log \left(\frac{\mu_{\left|1^{A, B}\right\rangle}^{2}}{2}\right)+1
\end{aligned}
$$

then,

$$
\begin{aligned}
\infty-2=\infty & =-\log \left(\frac{\mu_{\left|0^{A, B}\right\rangle}^{2}-\mu_{\left|1^{A, B}\right\rangle}^{2}}{4}\right)+\log \left(\frac{\mu_{\left|0^{A, B}\right\rangle}^{2}}{2}\right)+\log \left(\frac{\mu_{\left|1^{A, B}\right\rangle}^{2}}{2}\right) \\
& =\log \left(\frac{\frac{\mu_{\left|0^{A, B}\right\rangle}^{2}}{2} \frac{\mu_{\left|1^{A, B}\right\rangle}^{2}}{2}}{\frac{2}{\mu_{\left|0^{A, B}\right\rangle}^{2}-\mu_{\left|1^{A, B}\right\rangle}^{2}}}\right)=\log \left(\frac{\mu_{\left|0^{A, B}\right\rangle}^{2}}{\mu_{\left|0^{A, B}\right\rangle}^{2}-\mu_{\left|1^{A, B}\right\rangle}^{2}}\right)=\log \left(\mu_{\left|\Uparrow^{A} \Uparrow^{A B}\right\rangle}\right)=\log \left(\mu_{\left|\Uparrow^{A, B}\right\rangle}^{2}\right)
\end{aligned}
$$

Finally, the second-degree-equivalent spins will be

$$
\begin{aligned}
\mu_{\left|\Uparrow^{A} \Uparrow^{B}\right\rangle} & =\mu_{\left|\Uparrow^{A, B}\right\rangle}^{2}=\frac{\mu_{\left|0^{A, B}\right\rangle}^{2} \mu_{\left|1^{A, B}\right\rangle}^{2}}{\mu_{\left|0^{A, B}\right\rangle}^{2}-\mu_{\left|1^{A, B}\right\rangle}^{2}}=\frac{\mu_{\left|0^{A} 0^{B}\right\rangle}\left(-\mu_{\left|1^{A} 1^{B}\right\rangle}\right)}{\mu_{\left|0^{A} 0^{B}\right\rangle}-\left(-\mu_{\left|1^{A} 1^{B}\right\rangle}\right)}=\frac{-\mu_{\left|0^{A} 0^{B}\right\rangle} \mu_{\left|1^{A} 1^{B}\right\rangle}}{\mu_{\left|0^{A} 0^{B}\right\rangle}+\mu_{\left|1^{A} 1^{B}\right\rangle}} \\
& =\mu_{\left|0^{A, B}\right\rangle}^{2} / / \mu_{\left|1^{A, B}\right\rangle}^{2}=\mu_{\left|0^{A} 0^{B}\right\rangle} / /\left(-\mu_{\left|1^{A} 1^{B}\right\rangle}\right)=2^{\infty}=+\infty
\end{aligned}
$$

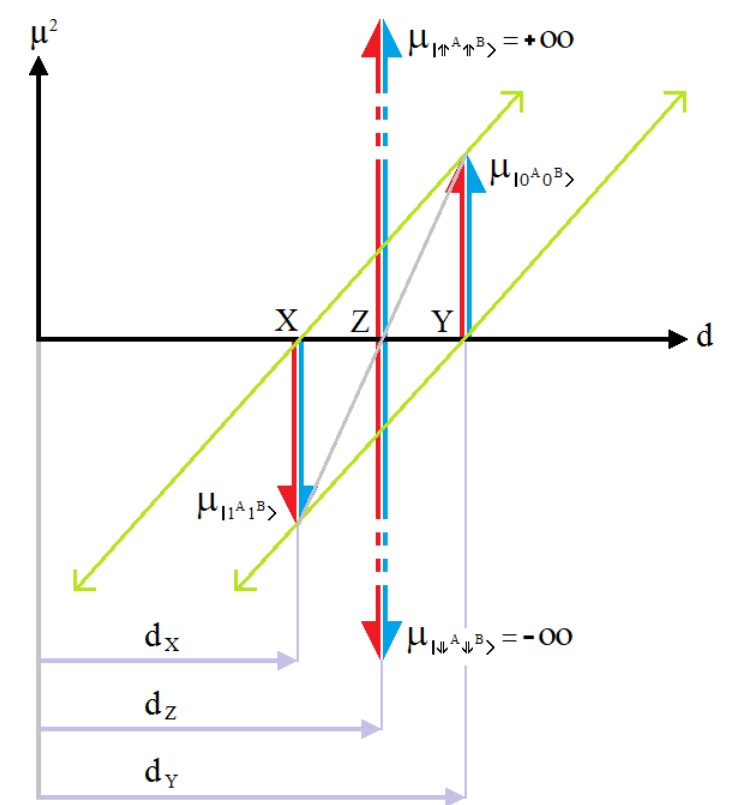

Figure 3.9 Parallel operator for entangled subsystems. 
Actually, equation (3.38) has two possible values, i.e., $\pm \infty$, which can be observed at both ends of Figure 3.9, where both light green lines are cut, and which connect the tip of $\mu_{\left|0^{A} 0^{B}\right\rangle}$ with the tail of $\mu_{\left|1^{A} 1^{B}\right\rangle}$ and the tip of $\mu_{\left|1^{A} 1^{B}\right\rangle}$ with the tail of $\mu_{\left|0^{A} 0^{B}\right\rangle}$. These results are shown in the equation (3.39)

$$
\left[\mu_{\left|\pi^{A} \pi^{B}\right\rangle}, \mu_{\left|\Downarrow^{A} \Downarrow^{B}\right\rangle}\right]=\left[\mu_{\left|\pi^{A, B}\right\rangle}^{2},-\mu_{\left|\Downarrow^{A, B}\right\rangle}^{2}\right]=[+\infty,-\infty]
$$

while their locations can be calculated thanks to equation (3.9). Such locations are determined graphically in Figure 3.9 by the intersection of the grey line, which connects the tip of both spins, with the thick black base line, i.e., axis $d$. Effectively, as we can see, equation (3.38) does not have a component or parameter with the positions of the original spins, in fact, this equation is insensitive to these locations; as a result, the second-degree-equivalent spins $\left(\mu^{2}\right)$ are not present where the light green lines are cut. Those crosses only allow us to determine their magnitudes. This is evident, since a spin could not have any projection on an axis of another nature as in the case of the axis of distance (d). Besides, as in the previous two cases (completely independent and classically correlated), both $\mu^{2}$ are together at an intermediate point determined by the equation (3.9) while the original spins are, in general, extremely far apart from each other. In fact, the exact locations of the original spins do not matter, what does matter is that $\mu^{2}$ will always be between them, since $\mu_{\left|0^{A} 0^{B}\right\rangle}=-\mu_{\left|1^{A} 1^{B}\right\rangle}$, therefore, equation (3.9) is simplified as equation (3.16). Summing up, the locations are an absolutely accessory subject for the spins, in other words, the problems between spins are dealt within the space of spins, directly and exclusively. Besides, the spin is always conserved, i.e., $\mu_{\left|0^{A} 0^{B}\right\rangle}+\mu_{\left|1^{A} 1^{B}\right\rangle}=0$, and, $\mu_{\left|\Uparrow^{A} \Uparrow^{B}\right\rangle}+\mu_{\left.\Downarrow^{A} \Downarrow^{B}\right\rangle}=0$.

Also, we will have two equivalent resulting spins for this level of correlation,

$$
\begin{aligned}
& \left|\Uparrow^{A} \Uparrow^{B}\right\rangle \equiv\left(\mu_{\left|\Uparrow^{A} \Uparrow^{B}\right\rangle}=\mu_{\left|\Uparrow^{A, B}\right\rangle}^{2}=+\infty\right) \\
& \left|\Downarrow^{A} \Downarrow^{B}\right\rangle \equiv\left(\mu_{\left|\Downarrow^{A} \Downarrow^{B}\right\rangle}=-\mu_{\left|\Downarrow^{A, B}\right\rangle}^{2}=-\infty\right)
\end{aligned}
$$

Here too,

$$
\begin{aligned}
& \left|0^{A} 0^{B}\right\rangle \equiv\left(\mu_{\left|0^{A} 0^{B}\right\rangle}=\mu_{\left|0^{A, B}\right\rangle}^{2}=+1\right) \\
& \left|1^{A} 1^{B}\right\rangle \equiv\left(\mu_{\left|1^{A} 1^{B}\right\rangle}=-\mu_{\left|1^{A, B}\right\rangle}^{2}=-1\right)
\end{aligned}
$$

If we move $\mu_{\left|0^{A} 0^{B}\right\rangle}$ from position Y to position $Y^{\prime}$, we will have $\mu_{\left|\Uparrow^{A} \Uparrow^{B}\right\rangle}=+\infty$ and $\mu_{\left|\Downarrow^{A} \Downarrow^{B}\right\rangle}=-\infty$ again. Besides, as in the case of completely independent subsystems, Figure 3.10 shows that $\mu_{\left|\Uparrow^{A} \Uparrow^{B}\right\rangle}$ and $\mu_{\left|\Downarrow^{A} \Downarrow^{B}\right\rangle}$ are absolutely insensitive to the distance between the original spins, as long as $\mu_{\left|0^{A} 0^{B}\right\rangle}$ and $\mu_{\left|1^{A} 1^{B}\right\rangle}$ do not change neither in modulus nor in its orientation (polarization). Regardless how much they separate from each other, such displacement does not alter the final result; in this case, $\mu_{\left|\Downarrow^{A} \Downarrow^{B}\right\rangle}=-\infty$ and $\mu_{\left|\Uparrow^{A} \Uparrow^{B}\right\rangle}=+\infty$. This confirms the success of the parallel operator since it attempts to model the entanglement, and as we know from several laboratory experiences, entanglement does not seem to care about the distance between the entangled particles [31, 49]. 


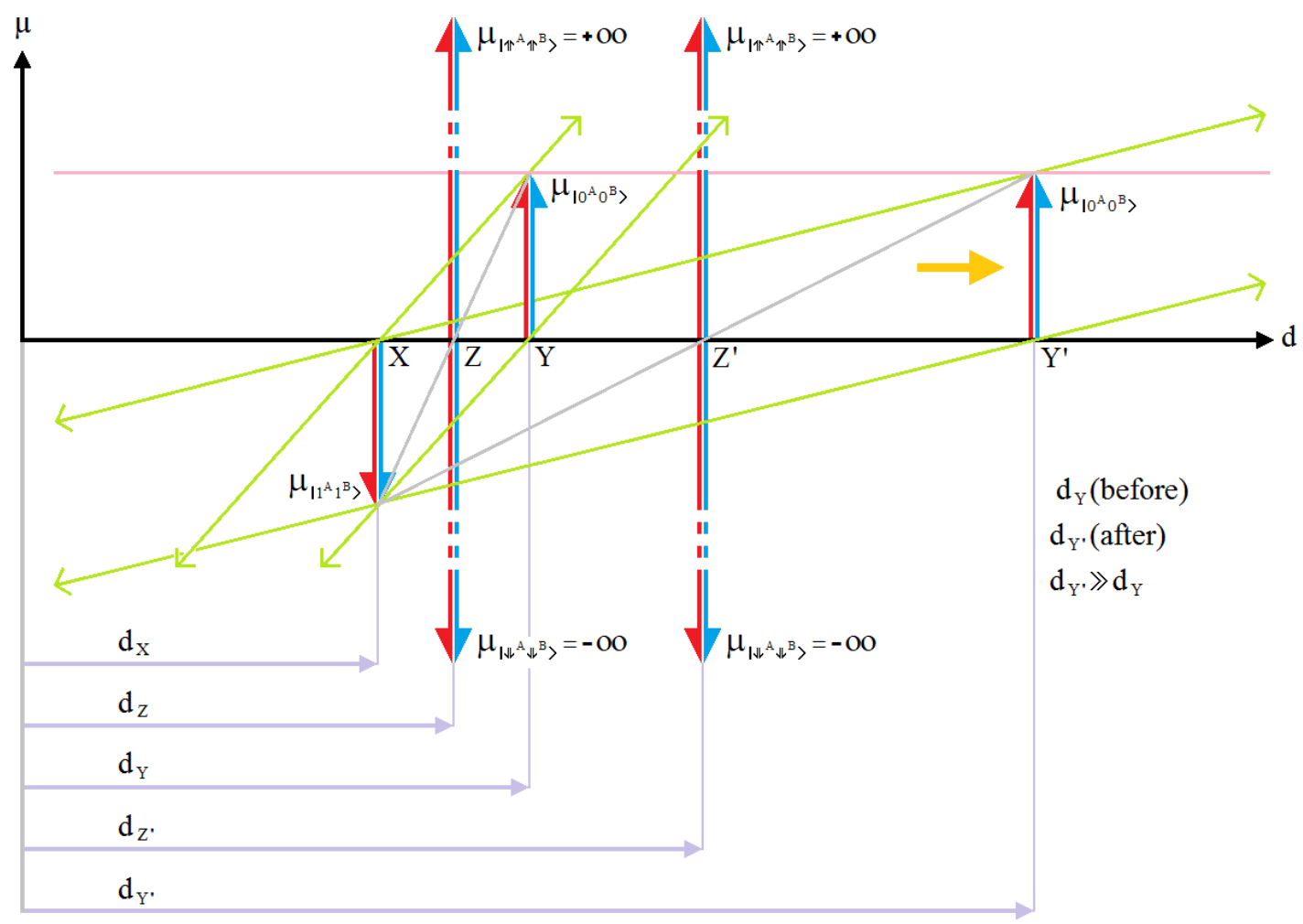

Figure 3.10 Parallel operator for entangled subsystems with an increase in the separation between the original spins.

On the other hand, if we properly apply square root to both sides of equation (3.38) and considering equation (3.40), then, we will obtain the four first-degree-equivalent spins $(\mu)$ or avatars. Then,

$$
\begin{gathered}
\mu_{\left|\Uparrow^{A, B}\right\rangle}^{2}=\frac{\mu_{\left|0^{A, B}\right\rangle}^{2} \mu_{\left|1^{A, B}\right\rangle}^{2}}{\left.\mu_{\left|0^{A, B}\right\rangle}^{2}-\mu_{\left|1^{A, B}\right\rangle}^{2}\right\rangle} \Rightarrow \mu_{\left|\pi^{A, B}\right\rangle}= \pm \sqrt{\frac{\left.\mu_{\left|0^{A, B}\right\rangle}^{2} \mu_{\left|1^{A, B}\right\rangle}^{2}\right\rangle}{\left.\mu_{\left|0^{A, B}\right\rangle}^{2}-\mu_{\left|1^{A, B}\right\rangle}^{2}\right\rangle}} \\
-\mu_{\left|\Downarrow^{A, B}\right\rangle}^{2}=-\frac{\left.\mu_{\left|0^{A, B}\right\rangle}^{2} \mu_{\left|1^{A, B}\right\rangle}^{2}\right\rangle}{\mu_{\left|0^{A, B}\right\rangle}^{2}-\mu_{\left|1^{A, B}\right\rangle}^{2}} \Rightarrow \mu_{\left|\|^{A, B}\right\rangle}= \pm \sqrt{\frac{\left.\mu_{\left|0^{A, B}\right\rangle}^{2} \mu_{\left|1^{A, B}\right\rangle}^{2}\right\rangle}{\mu_{\left|0^{A, B}\right\rangle}^{2}-\mu_{\left|1^{A, B}\right\rangle}^{2}}}
\end{gathered}
$$

Equations (3.42a) and (3.42b) are identical, and from them we will obtain four solutions

$$
\begin{aligned}
& \mu_{\left|\Uparrow^{A}\right\rangle}=\sqrt{\frac{\mu_{\left|0^{A, B}\right\rangle}^{2} \mu_{\left|1^{A, B}\right\rangle}^{2}}{\left.\mu_{\left|0^{A, B}\right\rangle}^{2}-\mu_{\left|1^{A, B}\right\rangle}^{2}\right\rangle}}=\frac{\mu_{\left|0^{A}\right\rangle}}{\sqrt{1-\left(\frac{\left.\mu_{\left|1^{A, B}\right\rangle}\right\rangle}{\left.\mu_{\left|0^{A, B}\right\rangle}\right\rangle}\right.}}=\mu_{\left|0^{A}\right\rangle} \gamma=+\infty \\
& \mu_{\left|\Downarrow^{A}\right\rangle}=-\sqrt{\frac{\mu_{\left|0^{A, B}\right\rangle}^{2} \mu_{\left|1^{A, B}\right\rangle}^{2}}{\left.\mu_{\left|0^{A, B}\right\rangle}^{2}-\mu_{\mid 1^{A, B}}^{2}\right\rangle}}=\frac{-\mu_{\left|0^{A}\right\rangle}}{\sqrt{1-\left(\frac{\left.\mu_{\left|1^{A, B}\right\rangle}\right\rangle}{\mu_{\left|0^{A, B}\right\rangle}}\right)^{2}}}=-\mu_{\left|0^{A}\right\rangle} \gamma=\mu_{\left|1^{A}\right\rangle} \gamma=-\infty
\end{aligned}
$$




$$
\begin{aligned}
& \mu_{\left|\Uparrow^{B}\right\rangle}=\sqrt{\frac{\mu_{\left|0^{A, B}\right\rangle}^{2} \mu_{\left|1^{A, B}\right\rangle}^{2}}{\mu_{\left|0^{A, B}\right\rangle}^{2}-\mu_{\left|1^{A, B}\right\rangle}^{2}}}=\frac{\mu_{\left|0^{B}\right\rangle}}{\sqrt{1-\left(\frac{\left.\mu_{\left|1^{A, B}\right\rangle}\right\rangle}{\left.\mu_{\left|0^{A, B}\right\rangle}\right\rangle}\right)^{2}}}=\mu_{\left|0^{B}\right\rangle} \gamma=+\infty \\
& \mu_{\left|\Downarrow^{B}\right\rangle}=-\sqrt{\frac{\mu_{\left|0^{A, B}\right\rangle}^{2} \mu_{\left|1^{A, B}\right\rangle}^{2}}{\left.\mu_{\left|0^{A, B}\right\rangle}^{2}-\mu_{\left|1^{A, B}\right\rangle}^{2}\right\rangle}}=\frac{-\mu_{\left|0^{B}\right\rangle}}{\sqrt{1-\left(\frac{\mu_{\left|1^{A, B}\right\rangle}}{\mu_{\left|0^{A, B}\right\rangle}}\right)^{2}}}=-\mu_{\left|0^{B}\right\rangle} \gamma=\mu_{\left|1^{B}\right\rangle} \gamma=-\infty
\end{aligned}
$$

where

$$
\gamma=\frac{1}{\sqrt{1-\left(\frac{v}{c}\right)^{2}}}=\frac{1}{\sqrt{1-\left(\frac{\mu_{\left|1^{A, B}\right\rangle}}{\mu_{\mid 0^{A, B}}}\right)^{2}}}=\frac{1}{\sqrt{1-\left(\frac{\mu_{\left.0^{A, B}\right\rangle}}{\mu_{\left|1^{A, B}\right\rangle}}\right)^{2}}}
$$

is the Lorentz factor [4], and since $\mu_{\left.||^{A, B}\right\rangle}^{2}=\mu_{\left|1^{A, B}\right\rangle}^{2}$, being $v$ a generic speed, and $c$ the speed of light, if $v=c$ (as it happens in this case) then $\gamma=\infty$. This shows us the relativistic nature of the avatars of equation (3.43). We will exploit this characteristic to the maximum in $\S 4, \S 5$ and $\S 6$.

As we can see in equation (2.12) of §2, the original spins conserve the spin. Doing the same analysis, the avatars also conserve it, i.e., $\mu_{\left|\pi^{A}\right\rangle}+\mu_{\left|\|^{A}\right\rangle}+\mu_{\left|\Uparrow^{B}\right\rangle}+\mu_{\left.\|^{B}\right\rangle}=0$, in fact, they will always do it, even if the original spins do not, as seen in others sections.

Moreover, the original spins and their avatars form a homothecy between them, i.e., this takes place inside each original-avatar pair. In this case, the avatars are the shadows of the original spins projected to the infinite with the same morphology (in this case, the orientation or polarization of the spins) and with infinite magnitude. See Figure 3.11.

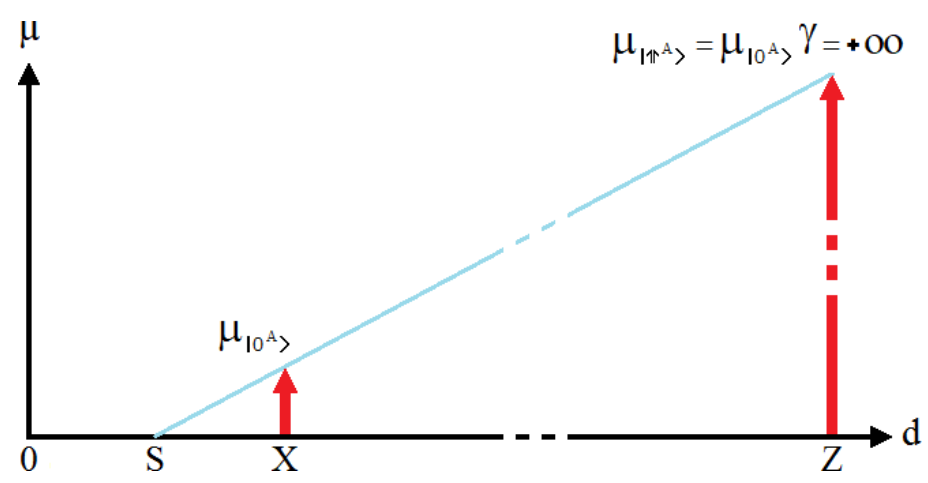

Figure 3.11 Homothetic transformation

As an example, Figure 3.11 shows us equation (3.43a), which is a homothetic transformation on the original spin $\mu_{\left|0^{4}\right\rangle}$. Unlike the case of Figure 3.4 for the completely independent case where each 
original spin is contracted in a $\zeta$ factor inasmuch as $\zeta=\sqrt{2} / 2<1$, in the entangled case each original spin is dilated in a proportion equal to the Lorentz factor for $v=c$, i.e., $\gamma=\infty$.
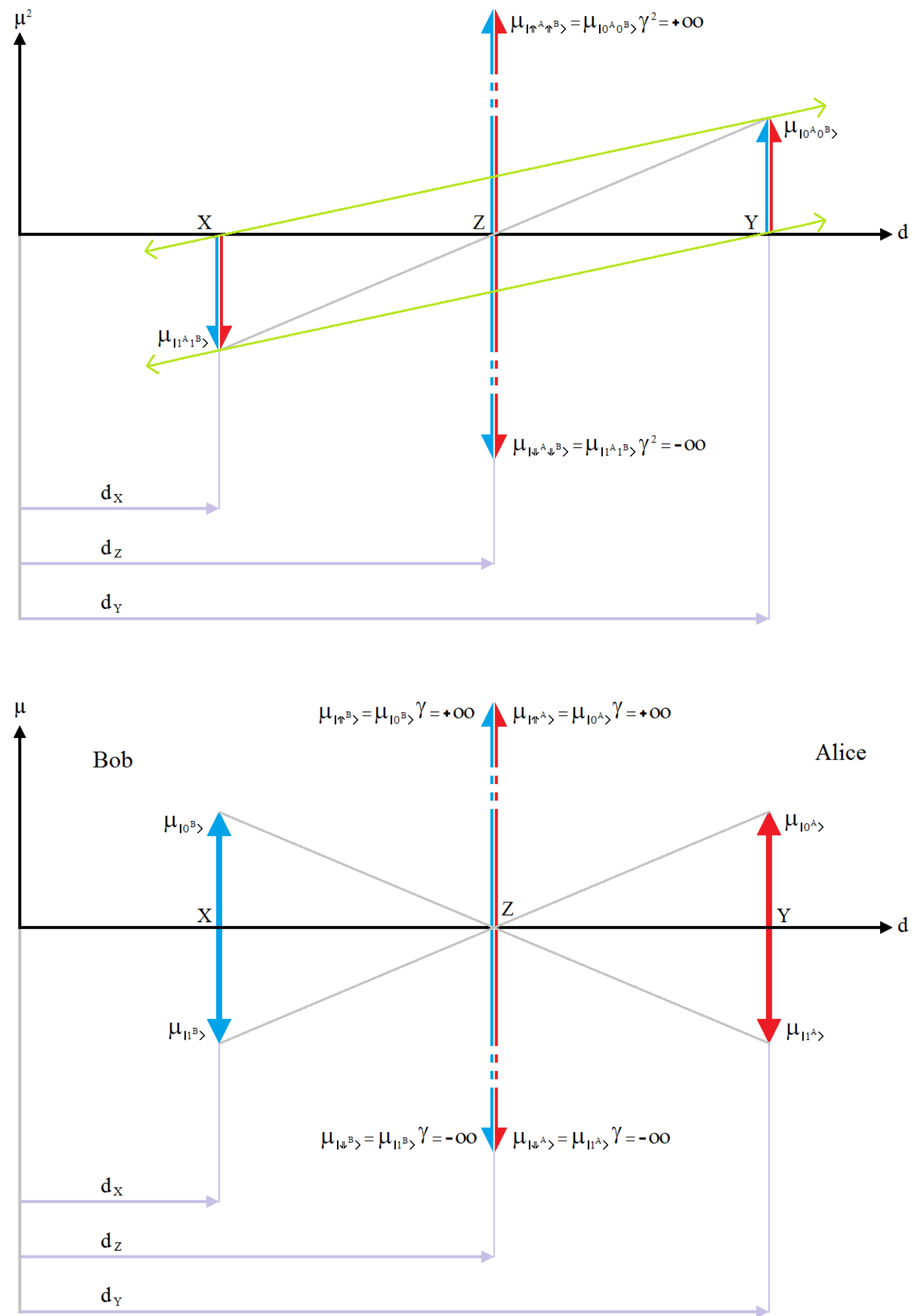

Figure 3.12 Second-degree-equivalent spin $\left(\mu^{2}\right)$ and first-degree $(\mu)$ or avatars, including distances for entangled subsystems. 
The upper part of Figure 3.12 shows us the second-degree-equivalent spins $\left(\mu^{2}\right)$ for entangled subsystems. We can highlight two equations of this figure,

$$
\begin{aligned}
& \mu_{\left|\pi^{A} \Uparrow^{B}\right\rangle}=\mu_{\left|0^{A} 0^{B}\right\rangle} \gamma^{2}=+\infty \\
& \mu_{\left|\Downarrow^{A} \Downarrow^{B}\right\rangle}=\mu_{\left|1^{A} 1^{B}\right\rangle} \gamma^{2}=-\infty
\end{aligned}
$$

while the lower part of Figure 3.12 shows the avatars with the same polarization and color of the original spin they represent, strictly according to each case of equation (3.43). An important aspect to highlight is that there are two second-degree-equivalent spins (equation 3.45 and upper part of Figure 3.12) and four avatars (equation 3.43 and lower part of Figure 3.12). Here also the avatars have identity and correspondence, that is, they are unambiguously associated with their corresponding original spin forming an indivisible original-avatar pair. Moreover, the original spins are inaccessible (since they are immersed in an integrating context such as a Bell base), therefore, the avatars are too. The point here is that avatars are equivalent to the original spins from the operational point of view. This will be seen in $\S 4$ and $\S 5$ in detail.

Final thoughts on this section:

- From the core of equation (3.34), i.e., from the scalar version of $S^{A \cup B}$, we obtained the avatars of equation (3.43). With the Dirac's notation based on 〈bra| and |ket>, we would have never deduced the avatars because with this notation, values, signs, polarizations, and orientations are blurred; which is inappropriate when working with spins, in particular, when we want to make all types of arithmetical operations between or with spins. In other words, this notation is inimical to the spins that are the spice of Physics. Then, we could say that the Dirac's notation is like a bull in a china shop.

- Equation (3.43) tells us that the four spins resulting from the entanglement or avatars suffer a dilatation respect to their originals according to the Lorentz's factor when $v=c$, i.e., $\gamma=\infty$ [4].

- The original spins and their avatars form a spatial homothecy between them, see equation (3.43), where the avatars are the shadows of the original spins projected to the infinite with the same morphology (in this case, the orientation or polarization of the spins) but with infinite magnitude.

- While the original spins are usually widely separated (even interplanetary distances), their avatars are always together at an intermediate point equidistant from the originals.

- Equation (3.43) shows the dual nature of avatars, which contains elements of both theories: GR and QM. For this reason, we say that the avatars are a hinge between them. Besides, we can see that this equation is a perfect balance between a spin (which is the fifth essence of QM) and the Lorentz's factor (which is a central tool in Relativity); i.e., since the entanglement is a tool which intrinsically belongs to the toolbox of the QM, it generates relativistic spins. In other words, we are talking about a relativistic nature of the entanglement in itself and in this way we make an approximation to the Theory of Everything (TOE) [20]. Somehow, this was mentioned in a work called: Dear Qubitzers, $\mathrm{GR}=\mathrm{QM}$ [71], which will be explored in more detail in $\$ 6.1$ along with ER=EPR conjecture [17-19].

- The lower part of Figure 3.12 clearly shows us what we have been saying about avatars being all together in a single intermediate point, i.e., they are local to each other even though the original spins are not. Then, when the avatars communicate with each other they will do so in an instantaneous way. In the next sections, we are going to see how the avatars are the operative representatives of the original spins, that is, the original spins are expressed through the avatars. Then, an instantaneous communication between avatars is equivalent to an instantaneous communication between the nonlocal original spins.

In $\S 4$ we will see two more ways to get to the equation (3.38), that is, to obtain the avatars. While in $\S 4, \S 5$, and $\S 6$ we will continue with the development of concepts related to the avatars as well as the nature, functioning, and interventions of entanglement in different fields. 


\subsection{Quasi-entangled subsystems}

Next, we will do the same analysis of the last subsection but when the original spins have different values. This case is represented in Figure 3.13.

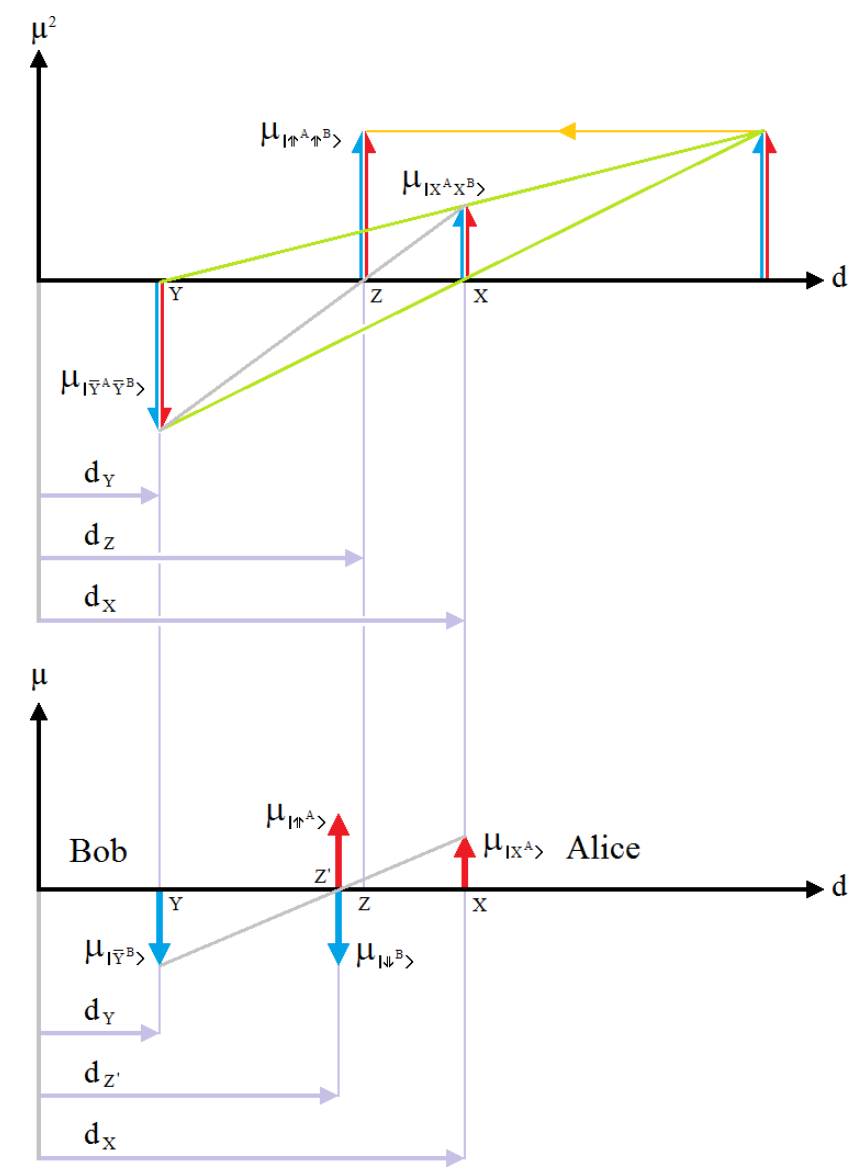

Figure 3.13 Quasi-entanglement between original spins with different values.

The upper part of Figure 3.13 shows that the second-degree-equivalent spin $\mu_{\left.\Uparrow^{\wedge} \Uparrow^{B}\right\rangle}$ arises from the intersections of the two light green lines. The former connects the tale of $\mu_{\left|\bar{Y}^{A} \bar{Y}^{B}\right\rangle}$ with the peak of $\mu_{\left|X^{A} X^{B}\right\rangle}$, and the latter connects the peak of $\mu_{\left|\bar{Y}^{A} \bar{Y}^{B}\right\rangle}$ with the tale of $\mu_{\left|X^{A} X^{B}\right\rangle}$. Unlike entanglement where we have two second-degree-equivalent spins, in quasi-entanglement, the value of $\mu_{\left.\Uparrow^{A} \Uparrow^{B}\right\rangle}$ is unique and also results from equation (3.38) where $\mu_{\left|\Uparrow^{A} \Uparrow^{B}\right\rangle}<<\infty$, while its location results from equation (3.9). As in all the previous cases, the value of $\mu_{\left.\pi^{A} \pi^{B}\right\rangle}$ resulting from the parallel operator of equation (3.38) is insensitive to the distance between original spins. The main characteristic of quasientanglement is that the spin is not preserved: $\mu_{\left|X^{A} X^{B}\right\rangle}+\mu_{\left|\bar{Y}^{A} \bar{Y}^{B}\right\rangle} \neq 0$. The lower part of Figure 3.13 shows that the avatars were obtained with a very similar configuration to the lower part of Figure 3.6 (for completely independent subsystems with original spins of different values). The position of the avatars can be calculated thanks to equation (3.15) and it does not match the position of the seconddegree-equivalent spin shown above. Finally, as in all the other cases, the avatars always conserve the spin, i.e., $\mu_{\left|\pi^{A}\right\rangle}+\mu_{\left|\|^{B}\right\rangle}=0$. 


\subsection{Teratological cases}

The analysis for the representation of the entanglement based on the parallel operator "//" predicts strange configurations which have not been tested in the laboratory yet, e.g., three spins oriented according to the same axis like: $\mu_{\left|0^{A} 0^{B}\right\rangle}=1,-\mu_{\left|1^{A} 1^{B}\right\rangle}=1, \mu_{\left|\Psi^{E}\right\rangle}=-1 / 2$. See Figure 3.14. The first two are completely independent although they are linked according to the analysis of $\S 3.1$, and together they are entangled with the third one, which is an isolated particle in yellow. This case does not comply with the conservation of spin, since: $1+1-1 / 2 \neq 0$.

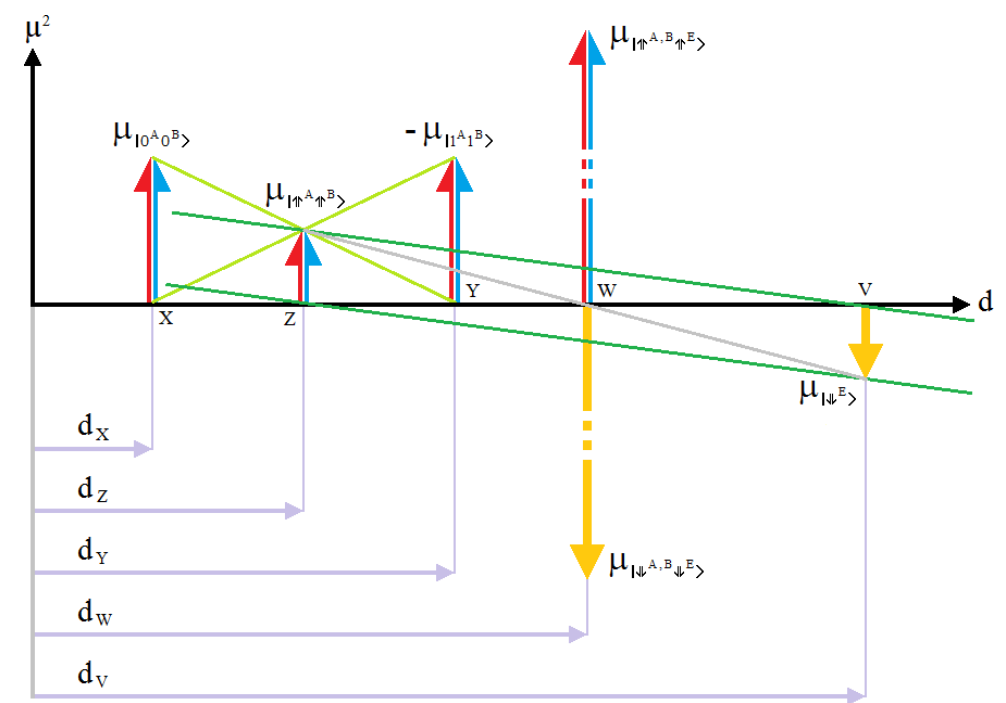

Figure 3.14 Two completely independent spins $\left(\mu_{\left|0^{A} 0^{B}\right\rangle}\right.$ and $\left.\mu_{\left|1^{A} 1^{B}\right\rangle}\right)$ are entangled with a third isolated spin $\mu_{\left|\Downarrow^{E}\right\rangle}$.

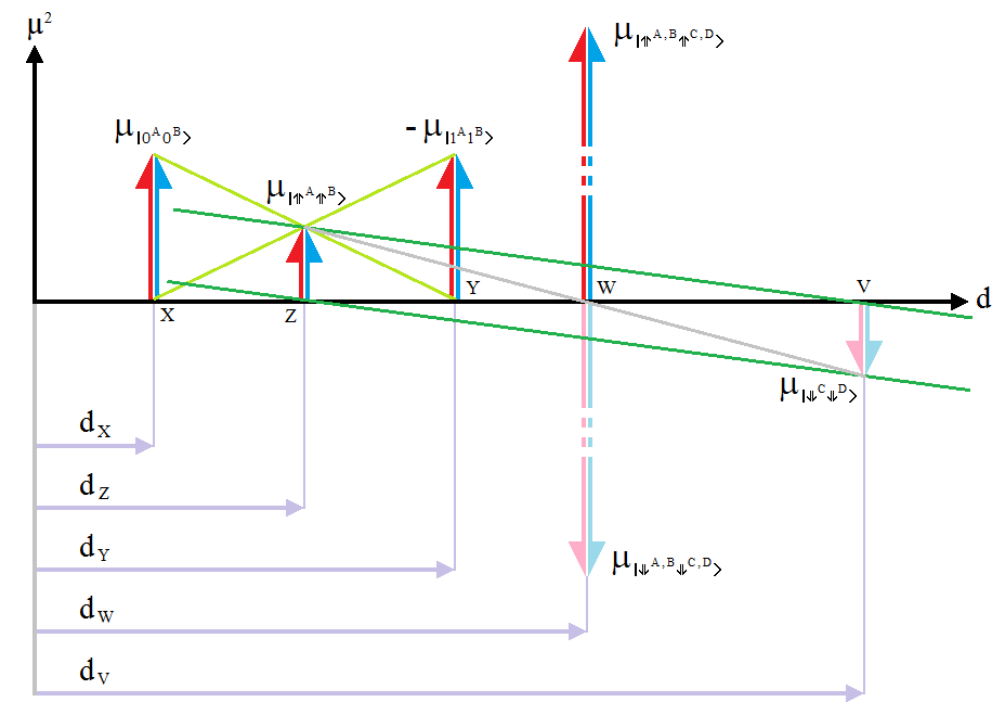

Figure 3.15 Two completely independent spins $\left(\mu_{\left|0^{A} 0^{B}\right\rangle}\right.$ and $\mu_{\left|1^{A} 1^{B}\right\rangle}$ ) are entangled with the result of another two completely independent spins, i.e., $\mu_{\left.\Downarrow \Downarrow^{C} \Downarrow^{D}\right\rangle}$.

Figure 3.15 shows the entanglement between two pairs of completely independent particles. In this case, the spin is preserved: $+1 / 2-1 / 2=0$. It would be interesting to see the comparison between this case with the 4-partite configuration that we will see in \$5.2. Finally, literature about entanglement, curiously, does not mention anything about it, maybe because it does not exist in reality. 


\section{Living with avatars}

This section is dedicated to refining some concepts discussed in $\S 3$. This will allow us to give a better conceptual dimension to these concepts for a more accurate approach to $\S 5$ and $\S 6$.

\subsection{Parallel operator "//"}

This operator is present in all Physics, and which we can find in:

- Masses of the nuclei of some diatomic molecules [5]

- Reactive circuits RLC as a combination of: resistance (R), inductor (L) and capacitor (C) [72, 73]

- Lens, in the famous lens equation [74-77]

- Wavelengths resulting from a non-linear crystal down conversion [78]

- Among many others

Here, we will only mention a couple of them and superficially. Next, we will explore the first and fourth cases. Then, in some diatomic molecules, if classical physics were applicable, the nuclei would have energy [5],

$$
E_{\text {classical }}=\frac{p_{1}^{2}}{2 m_{1}}+\frac{p_{2}^{2}}{2 m_{2}}+\frac{1}{2} k x^{2}
$$

where $m_{1}$ and $m_{2}$ are the masses of the nuclei, $p_{1}$ and $p_{2}$ are the magnitudes of their respective momenta, $k$ is a constant and $x$ denotes displacement or distance. In the center-of-mass frame, we can set $p_{1}=p_{2}=p$ and, by introducing the reduced mass (resulting and equivalent mass) through the parallel operator, we will have

$$
\mu=\frac{m_{1} m_{2}}{m_{1}+m_{2}}=m_{1} / / m_{2}
$$

where $\mu$ only means reduced mass, and does not mean spin. Then, we will obtain

$$
E_{\text {classical }}=\frac{p^{2}}{2 \mu}+\frac{1}{2} k x^{2}
$$

It is clear that both masses $m_{1}$ and $m_{2}$ do not have any kind of correlation between them. This example of application of parallel operator refers to the case of $\S 3.1$ for completely independent particles. On the other hand, in a non-linear crystal, which is impacted by a laser in order to obtain an entangled pair, a small fraction of the laser photons decay into pairs of photons by the process of spontaneous parametric down conversion. Both resulting photons comply with the energy conservation, therefore,

$$
E_{l}=E_{h}+E_{v}
$$

where the subscripts mean: $l$ of laser beam, $h$ of the horizontal down converted photon, and $v$ of the vertical down converted photon, according to their polarization. Now, if

$$
k=\frac{E}{\hbar c} .
$$

Replacing equation (4.5) in each term of equation (4.4) and eliminating $\hbar c$ in all factors, we will have,

$$
k_{l}=k_{h}+k_{v} .
$$


Now, if

$k=\frac{2 \pi}{\lambda}$,

and replacing equation (4.7) into (4.6) and eliminating $2 \pi$ in all factors, we will have,

$\frac{1}{\lambda_{l}}=\frac{1}{\lambda_{h}}+\frac{1}{\lambda_{v}} \equiv \lambda_{l}=\frac{\lambda_{h} \lambda_{v}}{\lambda_{h}+\lambda_{v}}$.

In other words, the parallel operator was already present in the constitution of the entanglement itself long before the deduction of avatars. Besides, Table 4.1 shows some results of the parallel operator "//" employed in all mentioned equations so far, which allows us to better understand this operator from the arithmetic point of view.

Table 4.1. Some results of the parallel operator " $/ /$ ".

\begin{tabular}{cccccc}
\hline$/ /$ & 0 & $x$ & $\infty$ & $-x$ & $-\infty$ \\
\hline 0 & 0 & 0 & 0 & 0 & 0 \\
$x$ & 0 & $x / 2$ & $x$ & $\pm \infty$ & $x$ \\
$\infty$ & 0 & $x$ & $\infty$ & $-x$ & $\pm \infty$ \\
$-x$ & 0 & $\pm \infty$ & $-x$ & $-x / 2$ & $-x$ \\
$-\infty$ & 0 & $x$ & $\pm \infty$ & $-x$ & $-\infty$ \\
\hline
\end{tabular}

Where $x$ is a scalar and has a generic value.

Table 4.2 compares two attributes (quantity and size) of the resulting spin after applying the parallel operator " //" to the four viewed cases, depending on the degree of correlation between both original spins: completely independent, classically-correlated, entangled, and quasi-entangled. As we can see in Table 4.2, four resulting spins are generated in the cases of classically correlated and entangled spins only, while in the rest, we have two alone. Besides, the avatars have infinitely large modulus in the case of entanglement exclusively, whereas, in the other cases, they have finite modulus.

Table 4.2. Attributes (quantity and size) of the avatars resulting from applying the parallel operator "//" according to the degree of correlation between original spins.

\begin{tabular}{ccccc}
\hline attribute & independent & correlated & entangled & quasi-entangled \\
\hline quantity & 2 & 4 & 4 & 2 \\
size & finite & finite & infinite & finite \\
\hline
\end{tabular}

In the cases of completely independent and quasi-entangled spins, the resulting avatars have similar configuration as we can see in the lower part of Figures 3.5 and 3.13, respectively.

But, at this point, a question automatically arises: what does the resulting avatars represent physically for each of the 4 columns in Table 4.2? We are going to explore this in an ascendant order of importance. First, in the completely independent situation, the resulting spin is a kind of figurehead or representative of the population of the spins involved, without being in the least tied to any kind of correlation between them. In this case, the parallel operator is simply the instrument that conveys the finding of the avatars. This is exactly what happens to the masses and wavelengths discussed above. Second, for the classically-correlated and quasi-entanglement cases, the avatars are the witnesses of the correlation between the original spins. Third and most importantly, i.e., the case of entanglement, the avatars have a deeper (ontological) philosophical and physical meaning. As we will see in $\S 4.3$ the avatars are the ones that support the effect and the extraordinary attributes of entanglement. 


\subsection{Homothety}

In this section, we analyze in which other elements of the particle homothety is present [68] from the existence of the avatars, fundamentally, for the case of entanglement. For all the cases seen in $\S 3$, even in those where the original spins do not comply with the conservation of the spin, the avatars always comply with said conservation. In other words, the balance of the presence of avatars always closes to zero. However, and as a first impression the idea of the existence of spins of infinite value is usually a bit strong, but this idea is not new, in literature there are numerous works about particles with infinite spins [79-84], whose existence has not yet been discovered. There are also works on massless particles with infinite spins [83, 85-92] with identical considerations as the previous ones regarding their eventual existence, which can also be found under the name of massless continuous spin [82, 92-97], however, unfortunately, all these works are mere theoretical speculations. We must not forget the contribution of the Vasiliev Higher-Spin theory [98-103], which is a minimal extension of gravity with (massless, Gauge) fields of spin $\mathrm{s}>2$. It is a higher-derivative completion of gravity that has good chances of being a consistent theory of quantum gravity (without super-symmetries and extra dimensions). Besides, the idea of a relativistic spin [104-112] was not born with the present work, therefore, a relativistic entanglement [113-124] comes to light as a consequence of this relativis-tic spin. Moreover, in recent years numerous works have appeared linking entanglement with the GR [125-134] directly or indirectly, some of which pose an entanglement between black holes (giving rise to an ER bridge or wormhole, even if temporarily) which is clearly the ER = EPR conjecture [17-19] while others, instead, explore the relativistic consequences of entanglement.

But, returning to the main idea of homothecy and considering that the balance of the presence of the avatars always equals to zero, i.e., they are conserved, we are going to show that the homothety is also projected to other elements of each entangled particle. To achieve this, we resort to the Hamiltonian operator [51], which represents the total energy of the system and controls the evolution process. In most of the cases, the Hamiltonian is formed by kinetic and potential energy. However, if the particle is stationary, then, the kinetic energy is cancelled leaving the potential energy alone. In this case, it will be the only one to be linked to external forces applied to this particle. As a consequence of this, the control of the external forces is at the same time as the control of the evolution of the states of the system $[1,2,51-53]$. Then, suppose that the particle in question is polarized along the $z$ axis,

$$
H=c P_{z}=\omega S_{z},
$$

where $P_{z}$ and $S_{z}$ are the momentum and the spin matrices along the axis $z$, respectively, $\omega$ is the angular frequency, and $c$ is the speed of light, with

$$
\begin{aligned}
& P_{z}=\frac{\mu \sigma_{z} \hbar \omega}{c} \\
& S_{z}=\mu \sigma_{z} \hbar
\end{aligned}
$$

being $\sigma_{z}$ one of Pauli's matrices,

$$
\sigma_{x}=\left(\begin{array}{cc}
0 & 1 \\
1 & 0
\end{array}\right), \quad \sigma_{y}=\left(\begin{array}{cc}
0 & -i \\
i & 0
\end{array}\right), \quad \sigma_{z}=\left(\begin{array}{cc}
1 & 0 \\
0 & -1
\end{array}\right),
$$

$\hbar$ is the reduced Planck constant, i.e., $\hbar=h / 2 \pi$, and $\mu$ are the eigenvalues of the spin matrix $S_{z}$ (actually, of the $\sigma_{z}$ matrix), which will be $\mu= \pm 1$ in the case of photons and $\mu= \pm 1 / 2$ in the case of electrons [51], with positive sign for spin up and negative sign for spin down. Clearly, this coincides with the analysis based on the scalar notation of $\S 3$. Finally, 
$H=\mu \sigma_{z} \hbar \omega$

Therefore, we will obtain two values from equation (4.12),

$E_{H}= \pm|\mu| \hbar \omega$

according to the polarization of the different spins. But, from Special Theory of Relativity we know that the energy of a particle with speed $v=0$ is [4]

$E=m c^{2}$

although in the case of photons their speed is always $v=c$ and their energy $E=p c=\hbar k c=\hbar \omega$ with an apparently null mass [4]. Based on this set of equations, the same homothecy that affects the spins of each original-avatar pair among them, evidently also affects all other physical magnitudes of the particles involved in the entanglement. This can be seen in detail in Table 4.3.

Table 4.3. Projections of homothecy on the physical magnitudes of entangled particles.

\begin{tabular}{|c|c|c|c|c|}
\hline \multirow{2}{*}{ physical magnitude } & \multicolumn{2}{|c|}{ electrons $(v=0)$} & \multicolumn{2}{c|}{ photons } \\
\cline { 2 - 5 } & original & avatar & original & avatar \\
\hline spin $^{\mathrm{i}}$ & $\pm 1 / 2$ & $\pm 1 / 2 \gamma= \pm \infty$ & \pm 1 & $\pm 1 \gamma= \pm \infty$ \\
\hline mass $^{\mathrm{ii}}$ & $m_{e}$ & $\pm \gamma m_{e}= \pm \infty$ & 0 & 0 \\
\hline energy & $m_{e} c^{2}$ & $\pm \gamma m_{e} c^{2}= \pm \infty$ & $k \hbar c$ & $\pm k \hbar c \gamma= \pm \infty$ \\
\hline linear momentum & $m_{e} c$ & $\pm \gamma m_{e} c= \pm \infty$ & $k \hbar$ & $\pm k \hbar \gamma= \pm \infty$ \\
\hline ispin's row: the values \pm of the original spins represent the polarization of them \\
ii mass' row: $m_{e}$ (electron's mass) $=9.10938356 \times 10^{-31}$ kilograms
\end{tabular}

It is also evident that, all physical magnitude must be conserved between avatars, since these cannot appear on the radar and unbalance everything in the universe every time an experiment that involves entanglement is carried out in the laboratory. Therefore, spin, mass, energy, and linear momentum are conserved among avatars, i.e., in all cases the balance always gives zero. Of course, these demands do not reach the original entangled particles, with the exception for their spins.

Table 4.3 gives us much information open to all kind of conjectures, since, the mere idea of negative and positive masses being annulled and which belong to the avatars refers to the idea of antimatter. That is, the avatars behave as if they were the synthesis of all the antimatter necessary for entanglement to occur. That said, we now see the relationship between the elements of Hawking's radiation in the context of wormholes, which are present in the entanglement between two black holes and their relation to ER = EPR conjecture [17-19] as we will see in $§ 6.1$.

\subsection{Avatars sustain the effect}

Avatars are responsible for several extraordinary tasks:

- They represent the projection of the originals in the relativistic world.

- They are the plenipotentiary ambassadors of the original spins at the doors of the relativistic world with a particular responsibility when entanglement is applied in Quantum Communications, see \$6.2.

- They sustain the effect called entanglement. 
But, what does this last statement mean? Figure 4.1 shows us the four original spins and their four corresponding avatars resulting from an entanglement. If now, as an example, we calculate a pair of rates spin/distance-between-original-spins $\left(R_{\mu d_{B O S S}}\right)$ through which we will try to associate the deterioration of the effect based on the deterioration of this metric as we separate the original spins from each other, we will have,

$$
\begin{aligned}
& R_{\mu d_{B O S S}}\left(\mu_{\left|0^{A}\right\rangle}, 2 d_{Z Y}\right)=\frac{\mu_{\left|0^{A}\right\rangle}}{2 d_{Z Y}}=\frac{1}{2 d_{Z Y}}<<\infty \\
& R_{\mu d_{B O S S}}\left(\mu_{\left|\Uparrow^{A}\right\rangle}, d_{Z Z}\right)=\frac{\left.\mu_{\left|\pi^{A}\right\rangle}\right\rangle}{d_{Z Z}}=\frac{\infty}{0}=\infty
\end{aligned}
$$

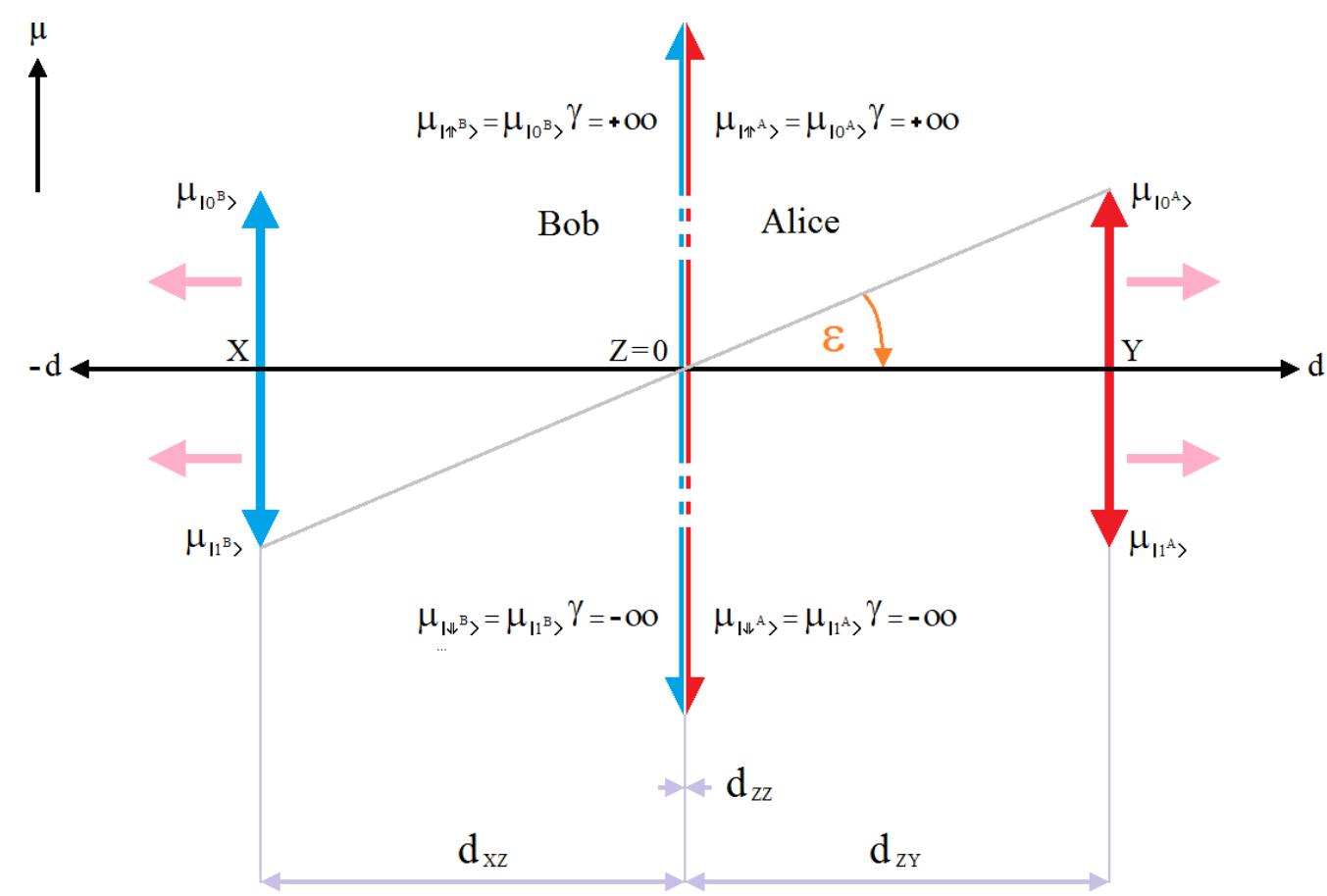

Figure 4.1 Rate spin/distance deteriorates in the original spins when moving away from each other, but remains intact in the avatars since they are all together in the same point.

where given that $d_{X Z}=d_{Z Y}$, then distance-between-original-spins $=2 d_{Z Y}$. The equation (4.15) represents the rate for one of the original spins, which is a finite result, while the equation (4.16) represents that rate for its corresponding avatar, which is infinite. The rate of avatars is formidable since there is no separation between them. If now, we separate the original spins as indicated by the pink arrows, both the distance $d_{X Z}$ and $d_{Z Y}$ tend to infinity while the angle $\varepsilon$ tends to zero, e.g.,

$$
\begin{aligned}
& \lim _{d_{Z Y} \rightarrow \infty} R_{\mu d_{B O S S}}\left(\mu_{\left|0^{A}\right\rangle}, 2 d_{Z Y}\right)=\lim _{d_{Z Y} \rightarrow \infty} \frac{\mu_{\left|0^{A}\right\rangle}}{2 d_{Z Y}}=\frac{1}{\infty}=0 \\
& \lim _{d_{Z Y} \rightarrow \infty} R_{\mu d_{B O S S}}\left(\mu_{\left|\Uparrow^{A}\right\rangle}, d_{Z Z}\right)=\lim _{d_{Z Y} \rightarrow \infty} \frac{\mu_{\left|\Uparrow^{A}\right\rangle}}{d_{Z Z}}=\frac{\infty}{0}=\infty
\end{aligned}
$$

That is to say, when $\mathrm{d}_{\mathrm{ZY}}$ tends to infinity, the rate $\mu_{\left|0^{A}\right\rangle} / d_{Z Y}$ of equation (4.15) tends to zero. But, 
what happens with the avatars? When $\mathrm{d}_{\mathrm{ZY}}$ tends to infinity (i.e., the original spins are infinitely separated), dzz continues to be zero since the avatars never separate, eventually they will move together but they will always share the same point. This tells us that its rate is insensitive to the separation of the original spins, therefore, it is evident that beyond the separation of the original spins, it is the avatars who sustain the effect. In other words, the rate of the original spins deteriorates due to their separation; however, the avatars' rates remain intact.

Without any ambition to send useful information through a link based entanglement (at least for now), and being simply the main objective to evaluate the reaction capacity of the entanglement about whether or not it is instantaneous and if that instantaneity collides with the GR [42-46] or rather, if a superluminal signaling [23-29] is needed for that purpose, then, the answer is:

- positive for the case of the original spins since the possibility of instant communication by being separated collides with the GR and, therefore, a superluminal signaling is needed for such a task. Instead, it is

- negative for the case of the avatars. There is no collision with the GR since being all the avatars stuck in a single point, any speed below the speed of light would allow us to go from one avatar to another instantly without any problem. In other words, between avatars there is no route or CHANNEL. Besides, since the original spins are expressed operatively through the avatars, and if the avatars are communicated instantaneously, then the original spins will also do so in this indirect or delegated way. Summing up, it is the avatars who really communicate, not the original spins. This will be seen in detail in $\$ 6.1, \$ 6.2$ and $\$ 6.3$.

\subsection{Alternative methods to obtain the avatars}

Next, we will develop two alternative methods to obtain the avatars.

The first one is taken from literature [135] where the Hamiltonian of the entanglement has a form like the following

$H=H^{A} \otimes I^{B}+I^{A} \otimes H^{B}$.

But, we also know [1] that a more consistent and complete model for entanglement is the following:

$H^{A \cup B}=H^{A} \otimes I^{B}+I^{A} \otimes H^{B}+H^{A \cap B}$,

where the interaction between both subsystems $A$ and $B$ is described by the Hamiltonian $H^{A \cap B} \neq 0$ so that each individual subsystem is an open quantum system [1]. The Hamiltonian as a whole, and under these circumstances, takes the form of equation (4.20). However, we must consider the following two fundamental aspects:

- the first aspect tells us about a greater approximation between the treatment based on entropy as well as that based on the Hamiltonian,

- and the second one is the possibility of working with a Hamiltonian model notably more simplified and, at the same time, closer to entanglement from the physical point of view, which is used with remarkable success $[1,136]$. Therefore, we understand that the best option is to use the following Hamiltonian,

$H^{A \cup B}=H^{A}+H^{B}+H^{A \cap B}=H^{A}+H^{B}+\frac{1}{H^{\prime \prime}} H^{A} H^{B}$,

where based on equation (4.12), we will have, 
$H^{A \cap B}=\frac{1}{H^{\prime \prime}} H^{A} H^{B}=\frac{\mu_{\left|0^{A} 0^{B}\right\rangle} \mu_{\left|1^{A} 1^{B}\right\rangle}}{\mu_{\left|\Uparrow^{A} \Uparrow^{B}\right\rangle}} \hbar \omega \sigma_{z}$

$H^{A}=\mu_{\left|0^{A} 0^{B}\right\rangle} \hbar \omega \sigma_{z}$

$H^{B}=\mu_{\left|1^{A} 1^{B}\right\rangle} \hbar \omega \sigma_{z}$

$H^{\prime \prime}=\mu_{\left|\Uparrow^{A} \Uparrow^{B}\right\rangle} \hbar \omega \sigma_{z}$

$H^{A \cup B}=\left(\mu_{\left|0^{A} 0^{B}\right\rangle}+\mu_{\left|1^{A} 1^{B}\right\rangle}\right) \hbar \omega \sigma_{z}=0$

Therefore, replacing equation (4.22) into (4.21) so that a pair of matrices $\sigma_{z}$ cancel each other before being replaced in $H^{A \cap B}$, we will have,

$0=\mu_{\left|0^{A} 0^{B}\right\rangle} \hbar \omega \sigma_{z}+\mu_{\left|1^{A} 1^{B}\right\rangle} \hbar \omega \sigma_{z}+\frac{\mu_{\left|0^{A} 0^{B}\right\rangle} \mu_{\left|1^{A} 1^{B}\right\rangle}}{\mu_{\left|\Uparrow^{A} \Uparrow^{B}\right\rangle}} \hbar \omega \sigma_{z}$.

Thus,

$\mu_{\left|\Uparrow^{A} \Uparrow^{B}\right\rangle}=\frac{-\mu_{\left|0^{A} 0^{B}\right\rangle} \mu_{\left|1^{A} 1^{B}\right\rangle}}{\mu_{\left|0^{A} 0^{B}\right\rangle}+\mu_{\left|1^{A} 1^{B}\right\rangle}}$

which coincides completely with equation (3.38).

The second alternative method is based on the entropy employed in $\$ 2.2$ and $\S 3$ to obtain the avatars which is also known as von Neumann's entropy which can be deduced as a limit case of the Rényi's entropy [137]. In fact, the $\alpha$ th order Rényi is defined as

$S_{\alpha} \equiv \frac{1}{1-\alpha} \log \left[\operatorname{Tr}\left(\rho^{\alpha}\right)\right]$

The zeroth-order $(\alpha=0)$ Rényi's entropy is related to the rank [137], namely, the number of nonzero singular values of $\rho$. When $\alpha \rightarrow 1$, the first-order Rényi's entropy reduces to the von Neumann's entropy,

$S_{1} \equiv-\operatorname{Tr}(\rho \log \rho)$

also known as Shannon's entropy which can also be represented as

$-\operatorname{Tr}(\rho \log \rho)=-\langle\log \rho\rangle$.

If we remember equation (3.32) and considering that for the scalar case

$-\langle\log \rho\rangle=-\log r$

then, replacing equation (4.28) into equation (3.32) in each case, we will have,

$S^{A \cap B}=S^{A}+S^{B}-S^{A \cup B}=-\log r^{A}-\log r^{B}+\log r^{A \cup B}$. 
Now, from equation (2.15) we can consider, among many other possibilities,

$$
\begin{aligned}
& r^{A}=\frac{1}{2}\left(\frac{\left.\mu_{\left|0^{A, B}\right\rangle}^{2}+\mu_{\left|1^{A, B}\right\rangle}^{2}\right)}{2}\right)=\frac{\mu_{\left|0^{A, B}\right\rangle}^{2}}{2}, \text { and } \\
& r^{B}=\frac{1}{2}\left(\frac{\mu_{\left|0^{A, B}\right\rangle}^{2}+\mu_{\left|1^{A, B}\right\rangle}^{2}}{2}\right)=\frac{\mu_{\left|1^{A, B}\right\rangle}^{2}}{2},
\end{aligned}
$$

given that $\mu_{\left|0^{A, B}\right\rangle}^{2}=\mu_{\left|1^{A, B}\right\rangle}^{2}=1$ for photons. If we replace equations (4.30) and (3.33) into equation (4.29), we will have,

$$
S^{A \cap B}=-\log \frac{\mu_{\left|0^{A, B}\right\rangle}^{2}}{2}-\log \frac{\mu_{\left|1^{A, B}\right\rangle}^{2}}{2}+\log \left(\frac{\left.\mu_{\left|0^{A, B}\right\rangle}^{2}-\mu_{\left|1^{A, B}\right\rangle}^{2}\right\rangle}{4}\right)^{2}
$$

If we make the appropriate additions and subtractions to equation (4.31), we will have:

$$
\begin{aligned}
S^{A \cap B} & =\log \frac{\mu_{\left|0^{A, B}\right\rangle}^{2}}{2}+\log \frac{\mu_{\left|1^{A, B}\right\rangle}^{2}}{2}-\log \left(\frac{\mu_{\left|0^{A, B}\right\rangle}^{2}-\mu_{\left|1^{A, B}\right\rangle}^{2}}{4}\right) \\
+ & {\left[-2 \log \frac{\mu_{\left|0^{A, B}\right\rangle}^{2}}{2}-2 \log \frac{\mu_{\left|1^{A, B}\right\rangle}^{2}}{2}+3 \log \left(\frac{\mu_{\left|0^{A, B}\right\rangle}^{2}-\mu_{\left|1^{A, B}\right\rangle}^{2}}{4}\right)\right] }
\end{aligned}
$$

If we send what is between brackets to the other side of the equal sign, then we obtain,

$$
\begin{gathered}
S^{A \cap B}+2 \log \frac{\mu_{\left|0^{A, B}\right\rangle}^{2}}{2}+2 \log \frac{\mu_{\left|1^{A, B}\right\rangle}^{2}}{2}-3 \log \left(\frac{\mu_{\left|0^{A, B}\right\rangle}^{2}-\mu_{\left|1^{A, B}\right\rangle}^{2}}{4}\right) \\
=\log \frac{\mu_{\left|0^{A, B}\right\rangle}^{2}}{2}+\log \frac{\mu_{\left|1^{A, B}\right\rangle}^{2}}{2}-\log \left(\frac{\mu_{\left|0^{A, B}\right\rangle}^{2}-\mu_{\left|1^{A, B}\right\rangle}^{2}}{4}\right)
\end{gathered}
$$

If we replace the corresponding values of some terms of the equation (4.33):

$$
S^{A \cap B}-2-2-3 \infty=\infty=\log \frac{\mu_{\left|0^{A, B}\right\rangle}^{2} \mu_{\left|1^{A, B}\right\rangle}^{2}}{\mu_{\left|0^{A, B}\right\rangle}^{2}-\mu_{\left|1^{A, B}\right\rangle}^{2}}=\log \frac{-\mu_{\left|0^{A} 0^{B}\right\rangle} \mu_{\left.||^{A} 1^{B}\right\rangle}}{\mu_{\left|0^{A} 0^{B}\right\rangle}+\mu_{\left|1^{A} 1^{B}\right\rangle}}=\log \mu_{\left|\Uparrow^{A} \Uparrow^{B}\right\rangle} .
$$

Finally, we obtain

$$
\mu_{\left|\Uparrow^{A} \Uparrow^{B}\right\rangle}=\frac{-\mu_{\left|0^{A} 0^{B}\right\rangle} \mu_{\left|1^{A} 1^{B}\right\rangle}}{\mu_{\left|0^{A} 0^{B}\right\rangle}+\mu_{\left|1^{A} 1^{B}\right\rangle}}
$$

which is exactly equal to equation (3.38). 
On the other hand, another version of the entanglement's Hamiltonian can be seen in [137]

$$
H_{e n t} \equiv-\log \rho
$$

which has a matrix structure. Then, if we consider its scalar version, we will have,

$S=h_{\text {ent }} \equiv-\log r$

As we can see, equation (4.37) completely matches with equation (4.28). The deduction starts again and ends with the same result, that is, equation (4.35).

In fact, there are three more methods that converge to the same result and that have been omitted in this paper due to space issues and because they are considered unnecessary. Such coincidences clearly indicate the consistency in the genesis of avatars.

\section{Avatars extend their domain}

In this section, we show that the analysis based on avatars fits perfectly with all the possibilities, versions and situations in which entanglement is involved, including decoherence [22, 138].

\subsection{Entanglement between particles that have never coexisted}

Figure 3.9 of $\$ 3.3$ shows us the typical case of entanglement, where the coordinates of point $X$ are $\left(d_{\mathrm{X}}, \mathrm{t}_{\mathrm{X}}\right)$, while those of point $\mathrm{Y}$ are $\left(d_{\mathrm{Y}}, \mathrm{t}_{\mathrm{Y}}\right)$, this means, they are present at the same instant but in a different space. However, the model allows an entanglement between spins that have never coexisted before [139]. In Figure 5.1, it can also be seen that the model is insensitive to inter-spin temporal separation as it is in the case of spatial separation. In this case, they are in the same space but at a different time since $\mathrm{X}$ is in the future and $\mathrm{Y}$ is in the past.

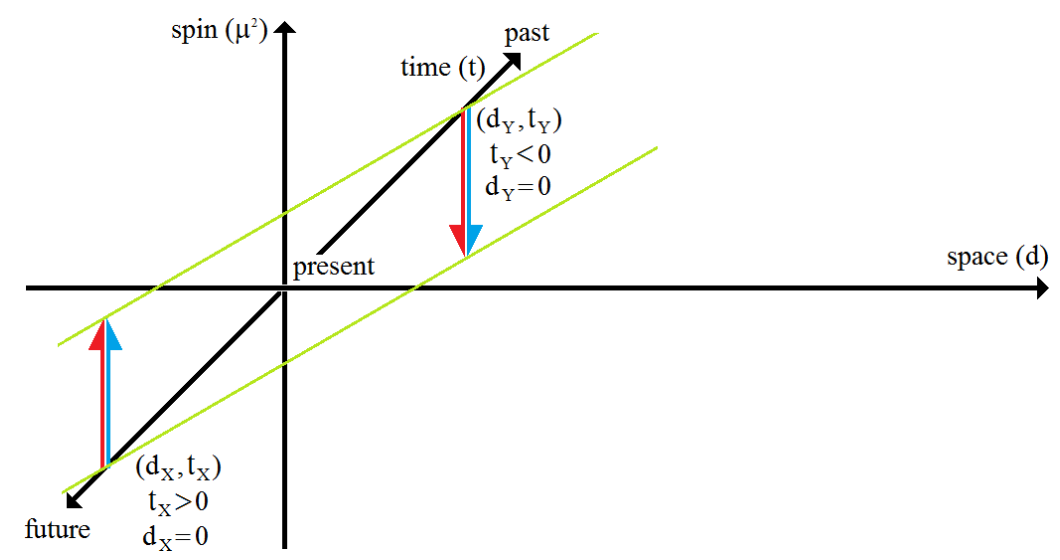

Figure 5.1 Entanglement between particles that have never coexisted before.

Finally, Figures 5.2 and $\mathbf{5 . 3}$ show us the spin $\mathrm{X}$ in the present in the position $d_{\mathrm{X}}$, while spin $\mathrm{Y}$ is in Figure 5.2 in the past and in Figure 5.3 in the future, in both cases in the position $d_{\mathrm{Y}}$. We can clearly conclude from the three figures in this subsection that the analysis based on avatars fits any spatiotemporal configuration in which the entanglement is involved.

It is this spatio-temporal ductility of avatars that is the winning card for its successful breakthrough in the analysis of the wormholes resulting from an entanglement process (WREP). The treatment of ER = EPR paradox, GR = QM and its application in Quantum Communications will be all analyzed in $\S 6$. 


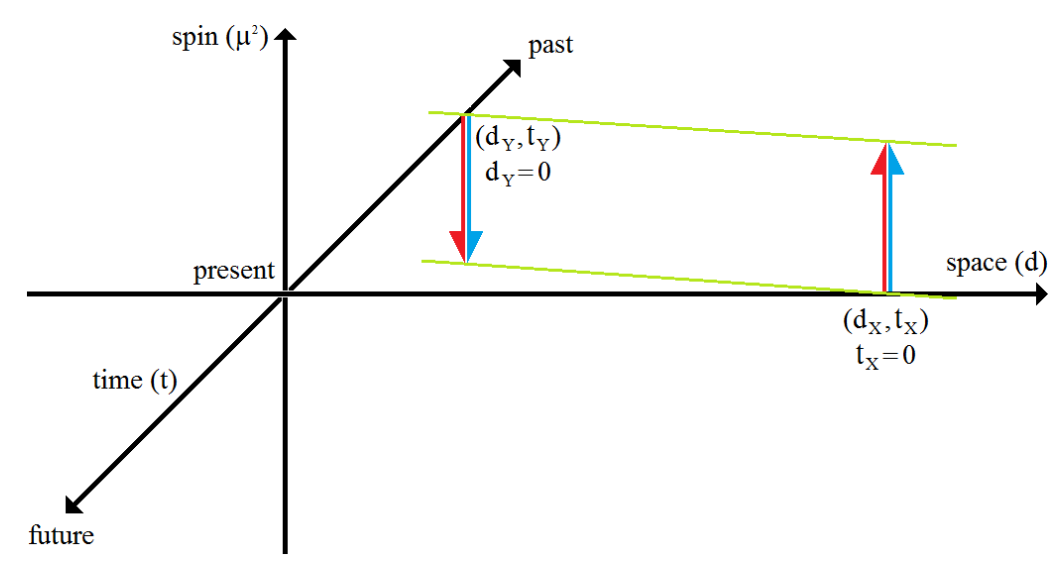

Figure 5.2 Particle $\mathrm{X}$ in the present in position $d_{\mathrm{X}}$, and particle $\mathrm{Y}$ in the past in position $d_{\mathrm{Y}}$.

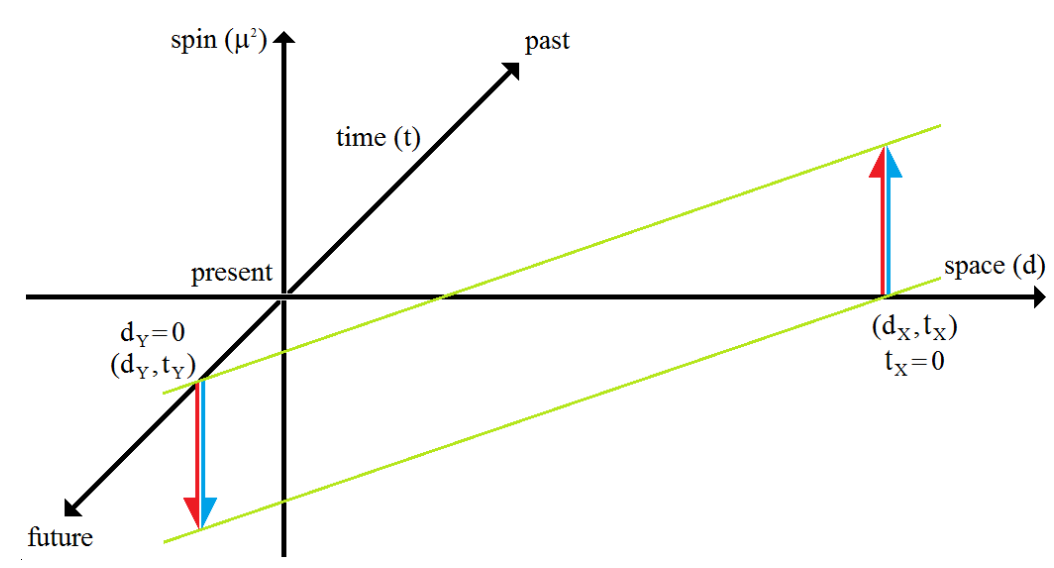

Figure 5.3 Particle $\mathrm{X}$ in the present in position $d_{\mathrm{X}}$, and particle $\mathrm{Y}$ in the future in position $d_{\mathrm{Y}}$.

\section{2. $N$-partite entanglement $(G H Z$ y W)}

In this section, six cases of entanglement will be analyzed according to the new perspective introduced by the analysis based on avatars: 2-partite, 3-partite [1,3] and 4-partite [3, 140] and more [141, 142] for GHZ and W states configurations. For this reason, we will introduce the complex root locus according to the canonicity of such state configurations [1]. The complex adjective has a double meaning:

- the root locus has a Hermitian nature, however,

- it represents the complexity of such root locus which depends on the $N$-partite degree and the type of state configuration: GHZ or W.

2-partite GHZ states configuration: Table 5.1 shows us this case in cell (2, GHZ). In fact, this is the case analyzed so far and on which all deductions were made. From equation (2.11) we have,

$\left|\Phi_{+}^{A \cup B}\right\rangle=\frac{1}{\sqrt{2}}\left(\left|0^{A}, 0^{B}\right\rangle+\left|1^{A}, 1^{B}\right\rangle\right)$.

In cell (2, GHZ) of Table 5.1, we can see a black ring with first-degree-original spins: $\left\{\mu_{\left|0^{A}\right\rangle}, \mu_{\left|1^{A}\right\rangle}\right\}$ in point $\mathrm{A}$, and $\left\{\mu_{\left|0^{B}\right\rangle}, \mu_{\left.1^{B}\right\rangle}\right\}$ in point $\mathrm{B}$, located at 180 degrees from each other. Now, if we remember the equation (3.38), we will have, 
Table 5.1. N-partite cases for GHZ and W states.

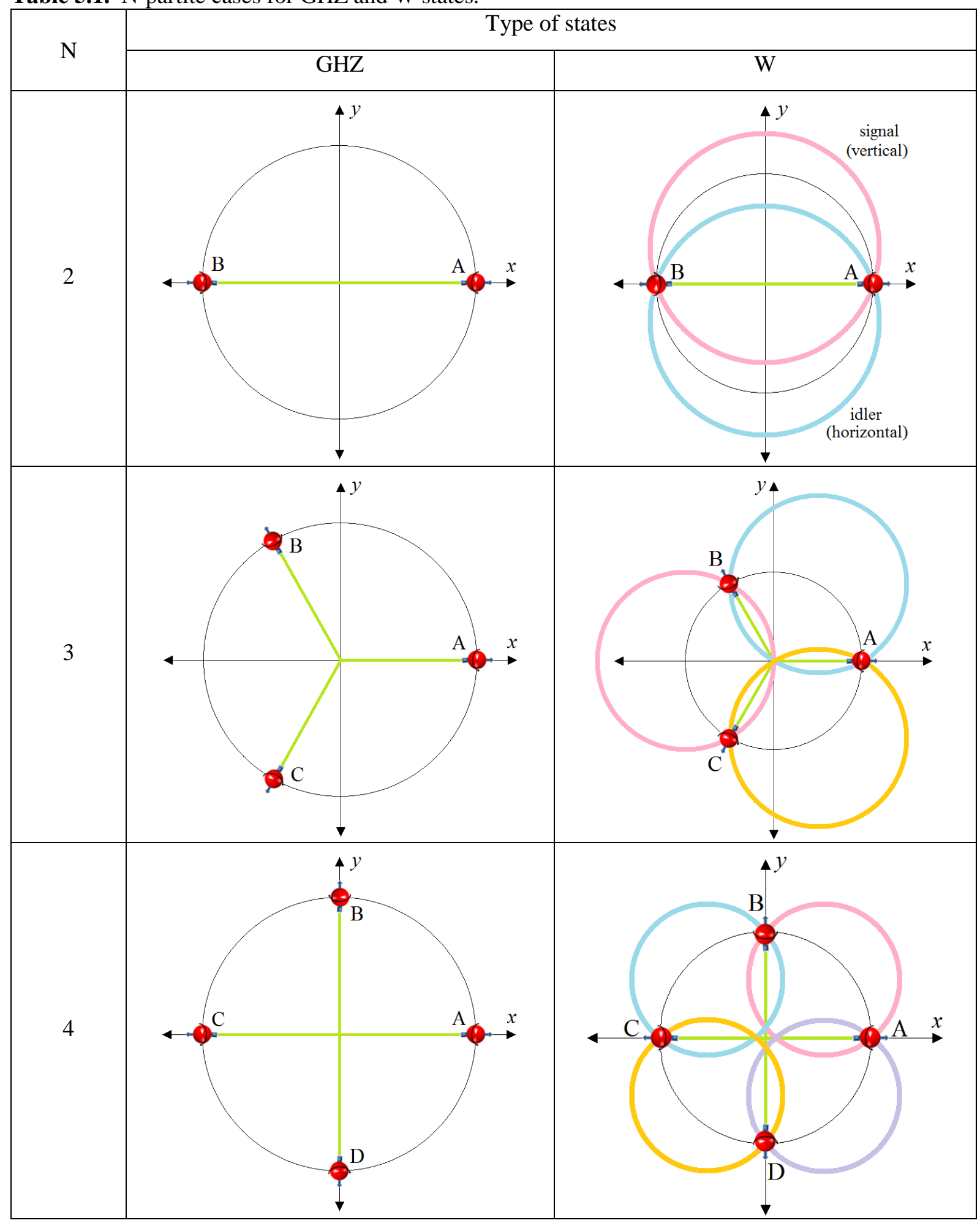

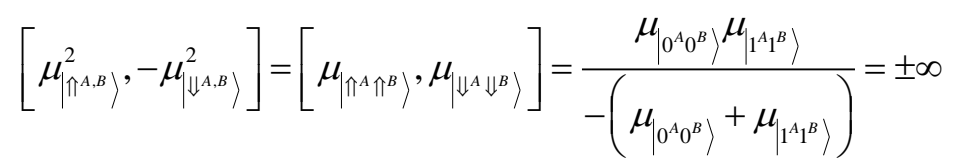

Denominator of equation (5.2) comply with the principle of spin conservation, and from this equation we can obtain the four avatars: $\mu_{\left|\Uparrow^{A}\right\rangle}, \mu_{\left|\Downarrow^{A}\right\rangle}, \mu_{\left|\Uparrow^{B}\right\rangle}$ and $\mu_{\left|\Downarrow^{B}\right\rangle}$ together in the center of the circle. 
3-partite GHZ states configuration: Table 5.1 shows this case in cell (3, GHZ), where everything increases in one degree with respect to the previous case, that is, the bases employed will be

$$
\left|\Phi_{+}^{A \cup B \cup C}\right\rangle=\frac{1}{\sqrt{2}}\left(\left|0^{A}, 0^{B}, 0^{C}\right\rangle+\left|1^{A}, 1^{B}, 1^{C}\right\rangle\right) .
$$

In cell (3, GHZ) of Table 5.1, we can see a black ring with first-degree-original spins $\left\{\mu_{\left|0^{A}\right\rangle}, \mu_{\left|1^{A}\right\rangle}\right\}$ in point $\mathrm{A},\left\{\mu_{\left|0^{B}\right\rangle}, \mu_{\left|1^{B}\right\rangle}\right\}$ in point $\mathrm{B}$, and $\left\{\mu_{\left|0^{C}\right\rangle}, \mu_{\left|1^{C}\right\rangle}\right\}$ in point $\mathrm{C}$, at 120 degrees from each other. Then, for this case, we will have,

$$
\left[\mu_{\left|\Uparrow^{A, B, C}\right\rangle}^{3},-\mu_{\left|\Downarrow^{A, B, C}\right\rangle}^{3}\right]=\left[\mu_{\left|\Uparrow^{A} \Uparrow^{B} \Uparrow^{C}\right\rangle}, \mu_{\left|\Downarrow^{A} \Downarrow^{B} \Downarrow^{C}\right\rangle}\right]=\frac{\mu_{\mid 0^{A} 0^{B} 0^{C}} \mu \mu_{\left.\left.\right|^{A} 1^{B} 1^{C}\right\rangle}}{-\left(\mu_{\left|0^{A} 0^{B} 0^{C}\right\rangle}+\mu_{\left|1^{A} 1^{B} 1^{C}\right\rangle}\right)}= \pm \infty
$$

Denominator of equation (5.4) also comply with the principle of spin conservation, and from this equation we can obtain the six avatars: $\mu_{\left|\Uparrow^{A}\right\rangle}, \mu_{\left|\Downarrow^{A}\right\rangle}, \mu_{\left|\Uparrow^{B}\right\rangle}, \mu_{\left|\Downarrow^{B}\right\rangle}, \mu_{\left|\Uparrow^{c}\right\rangle}$ and $\mu_{\left|\Downarrow^{C}\right\rangle}$ together in the center of the circle.

4-partite GHZ states configuration: Table 5.1 shows this case in cell (4, GHZ), where everything increases in one degree with respect to the previous case, thus, the bases employed will be

$$
\left|\Phi_{+}^{A \cup B \cup C \cup D}\right\rangle=\frac{1}{\sqrt{2}}\left(\left|0^{A}, 0^{B}, 0^{C}, 0^{D}\right\rangle+\left|1^{A}, 1^{B}, 1^{C}, 1^{D}\right\rangle\right)
$$

In cell (4, GHZ) of Table 5.1, we can see a black ring with first-degree-original spins $\left\{\mu_{\left|0^{A}\right\rangle}, \mu_{\left|1^{A}\right\rangle}\right\}$ in point $\mathrm{A},\left\{\mu_{\left|0^{B}\right\rangle}, \mu_{\left|1^{B}\right\rangle}\right\}$ in point $\mathrm{B},\left\{\mu_{\left|0^{C}\right\rangle}, \mu_{\left|1^{C}\right\rangle}\right\}$ in point $\mathrm{C}$, and $\left\{\mu_{\left|0^{D}\right\rangle}, \mu_{\left|1^{D}\right\rangle}\right\}$ in point $\mathrm{D}$, at 90 degrees from each other. Then, for this case, we will have,

$$
\left[\mu_{\left|\Uparrow^{A, B, C, D}\right\rangle}^{4},-\mu_{\left|\Downarrow^{A, B, C, D}\right\rangle}^{4}\right]=\left[\mu_{\left|\Uparrow^{A} \Uparrow^{B} \Uparrow^{C} \Uparrow^{D}\right\rangle}, \mu_{\left|\Downarrow^{A} \Downarrow B \Downarrow C \Downarrow^{D}\right\rangle}\right]=\frac{\mu_{\left|0^{A} 0^{B} 0^{C} 0^{D}\right\rangle} \mu_{\left|1^{A} 1^{B} 1^{C} 1^{D}\right\rangle}}{-\left(\mu_{\left|0^{A} 0^{B} 0^{C} 0^{D}\right\rangle}+\mu_{\left|1^{A} 1^{B} C^{D} 1^{D}\right\rangle}\right)}= \pm \infty
$$

Denominator of equation (5.6) also comply with the principle of spin conservation, and from this equation we can obtain the eight avatars: $\mu_{\left|\Uparrow^{A}\right\rangle}, \mu_{\left|\Downarrow^{A}\right\rangle}, \mu_{\left|\Uparrow^{B}\right\rangle}, \mu_{\left|\Downarrow^{B}\right\rangle}, \mu_{\left|\Uparrow^{C}\right\rangle}, \mu_{\left|\Downarrow^{C}\right\rangle}, \mu_{\left|\Uparrow^{D}\right\rangle}$ and $\mu_{\left|\Downarrow^{D}\right\rangle}$ together in the center of the circle.

2-partite W states configuration: Table 5.1 shows this case in cell (2, W). From equation (2.11) we have,

$$
\left|\Psi_{+}^{A \cup B}\right\rangle=\frac{1}{\sqrt{2}}\left(\left|0^{A}, 1^{B}\right\rangle+\left|1^{A}, 0^{B}\right\rangle\right)
$$

In cell (2, W) of Table 5.1, we can see the points A and B in the intersections of the pink and light 
blue rings. An equivalent black ring contains the first-degree-original spins $\left\{\mu_{\left|0^{A}\right\rangle}, \mu_{\left|1^{A}\right\rangle}\right\}$ in point A, and $\left\{\mu_{\left|0^{B}\right\rangle}, \mu_{\left.1^{B}\right\rangle}\right\}$ in point $\mathrm{B}$, at 180 degrees from each other. Then, for this case, we will have,

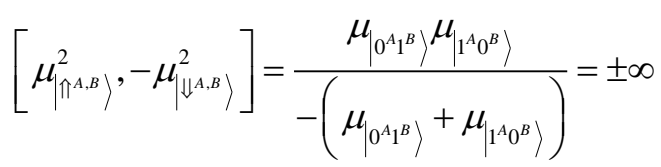

Denominator of equation (5.8) comply with the principle of spin conservation, and from this equation we can obtain the four avatars: $\mu_{\left|\Uparrow^{A}\right\rangle}, \mu_{\left|\Downarrow^{A}\right\rangle}, \mu_{\left|\Uparrow^{B}\right\rangle}$ and $\mu_{\left|\Downarrow^{B}\right\rangle}$ together in the center of the black circle.

3-partite W states configuration: Table 5.1 shows this case in cell $(3, \mathrm{~W})$, where everything increases in one degree with respect to the previous case, then, the bases employed will be

$$
\left|\Psi_{+}^{A \cup B \cup C}\right\rangle=\frac{1}{\sqrt{3}}\left(\left|1^{A}, 0^{B}, 0^{C}\right\rangle+\left|, 0^{A}, 1^{B}, 0^{C}\right\rangle+\left|0^{A}, 0^{B}, 1^{C}\right\rangle\right)
$$

In cell $(3, \mathrm{~W})$ of Table 5.1, we can see three points: A in the intersection of the orange and light blue rings, $\mathrm{B}$ in the intersection of the light blue and pink rings, and $\mathrm{C}$ in the intersection of the pink and orange rings, respectively. Here too, an equivalent black ring contains the first-degree-original spins $\left\{\mu_{\left|0^{A}\right\rangle}, \mu_{\left|1^{A}\right\rangle}\right\}$ in point $\mathrm{A},\left\{\mu_{\left|0^{B}\right\rangle}, \mu_{\left|1^{B}\right\rangle}\right\}$ in point $\mathrm{B}$, and $\left\{\mu_{\left|0^{C}\right\rangle}, \mu_{\left|1^{C}\right\rangle}\right\}$ in point $\mathrm{C}$, at 120 degrees from each other. Then, for this case, we will have,

$$
\left[\mu_{\left|\pi^{A, B, C}\right\rangle}^{3},-\mu_{\left|\Downarrow^{A, B, C}\right\rangle}^{3}\right]=\frac{\mu_{\left|1^{A} 0^{B} 0^{C}\right\rangle} \mu_{\left|0^{A} 1^{B} 0^{C}\right\rangle} \mu_{\left|0^{A} 0^{B} 1^{C}\right\rangle}}{-\left(\mu_{\left|1^{A} 0^{B} 0^{C}\right\rangle} \mu_{\left|0^{A} 1^{B} 0^{C}\right\rangle}+\mu_{\left|1^{A} 0^{B} 0^{C}\right\rangle} \mu_{\left|0^{A} 0^{B} 1^{C}\right\rangle}+\mu_{\left|0^{A} 1^{B} 0^{C}\right\rangle} \mu_{\left|0^{A} 0^{B} 1^{C}\right\rangle}\right)}= \pm \infty
$$

Denominator of equation (5.10) also comply with the principle of spin conservation, and from this equation we can obtain the six avatars: $\mu_{\left|\Uparrow^{A}\right\rangle}, \mu_{\left|\Downarrow^{A}\right\rangle}, \mu_{\left|\Uparrow^{B}\right\rangle}, \mu_{\left|\Downarrow^{B}\right\rangle}, \mu_{\left|\Uparrow^{C}\right\rangle}$ and $\mu_{\left|\Downarrow^{C}\right\rangle}$ together in the center of the black circle.

4-partite W states configuration: Table 5.1 shows this case in cell $(4, \mathrm{~W})$, where everything increases in one degree with respect to the previous case, thus, the bases employed will be

$$
\left|\Psi_{+}^{A \cup B \cup C \cup D}\right\rangle=\frac{1}{\sqrt{4}}\left(\left|1^{A}, 0^{B}, 0^{C}, 0^{D}\right\rangle+\left|, 0^{A}, 1^{B}, 0^{C}, 0^{D}\right\rangle+\left|0^{A}, 0^{B}, 1^{C}, 0^{D}\right\rangle+\left|0^{A}, 0^{B}, 0^{C}, 1^{D}\right\rangle\right) .
$$

In cell (4, W) of Table 5.1, we can see four points: A in the intersection of the violet and pink rings, B in the intersection of the pink and light blue rings, $\mathrm{C}$ in the intersection of the light blue and orange rings, and $\mathrm{D}$ in the intersection of the orange and violet rings, respectively. Here too, an equivalent black ring contains the first-degree-original spins: $\left\{\mu_{\left|0^{A}\right\rangle}, \mu_{\left|1^{A}\right\rangle}\right\}$ in point $\mathrm{A},\left\{\mu_{\left|0^{B}\right\rangle}, \mu_{\left|1^{B}\right\rangle}\right\}$ in point $\mathrm{B}$, $\left\{\mu_{\left|0^{C}\right\rangle}, \mu_{\left|1^{C}\right\rangle}\right\}$ in point $\mathrm{C}$, and $\left\{\mu_{\left|0^{D}\right\rangle}, \mu_{\left.1^{D}\right\rangle}\right\}$ in point $\mathrm{D}$, at 90 degrees from each other. Then, for this case, we will have, 


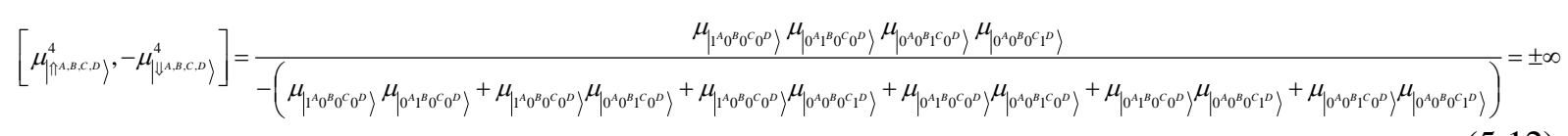

Denominator of equation (5.12) also comply with the principle of spin conservation, and from this equation we can obtain the eight avatars: $\mu_{\left|\Uparrow^{A}\right\rangle}, \mu_{\left|\Downarrow^{A}\right\rangle}, \mu_{\left|\Uparrow^{B}\right\rangle}, \mu_{\left|\Downarrow^{B}\right\rangle}, \mu_{\left|\Uparrow^{C}\right\rangle}, \mu_{\left|\Downarrow^{C}\right\rangle}, \mu_{\left|\Uparrow^{D}\right\rangle}$ and $\mu_{\left|\Downarrow^{D}\right\rangle}$ together in the center of the black circle.

\section{3. $N$-dimensional entanglement}

The W and GHZ states configuration [1] admits entanglement in $N$ spatial dimensions, i.e., not with respect to a unique dimension but projected on $N$ axes, as long as the entangled spins are always on a same plane. For example, we can see this in Figure 5.4 for 2 dimensions; however, the analysis is easily extendable to $N$ dimensions [143].

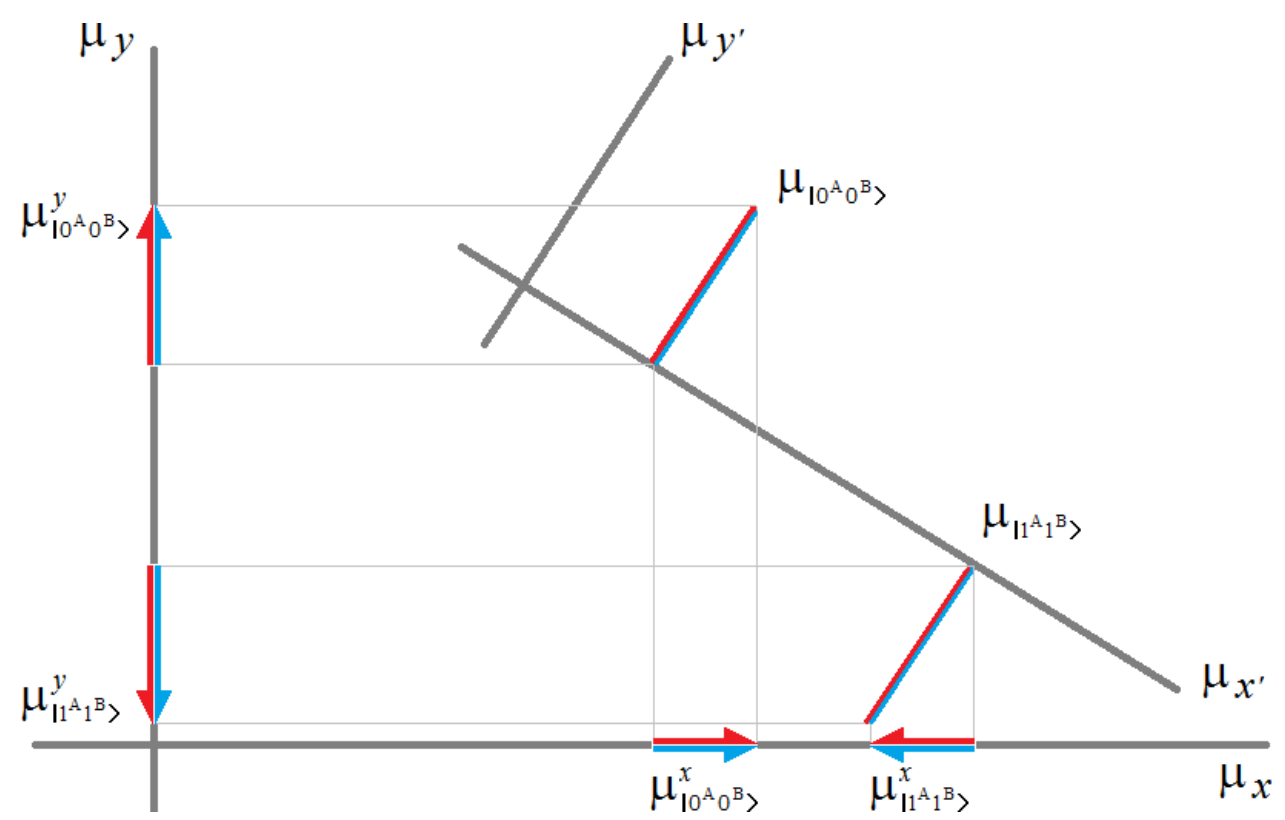

Figure 5.4 2-partite entanglement regarding 2 spatial axes.

Figure 5.4 shows us two entangled original spins $\mu_{\left|0^{A} 0^{B}\right\rangle}$ and $\mu_{\left|1^{A} 1^{B}\right\rangle}$, with projection on one of their original axis $\left(x^{\prime}, y^{\prime}\right): y^{\prime}$, however, they have projections on two new axes $(x, y)$, therefore, for their original axes the sum of spins will be,

$\mu_{\left|0^{A} 0^{B}\right\rangle}+\mu_{\left|1^{A} 1^{B}\right\rangle}=\left(\mu_{\left|0^{A} 0^{B}\right\rangle}^{x^{\prime}}+\mu_{\left|0^{A} 0^{B}\right\rangle}^{y^{\prime}}\right)+\left(\mu_{\left|1^{A} 1^{B}\right\rangle}^{x^{\prime}}+\mu_{\left|1^{A} 1^{B}\right\rangle}^{y^{\prime}}\right)=\left(\mu_{\left|0^{A} 0^{B}\right\rangle}^{x^{\prime}}+\mu_{\left|1^{A} 1^{B}\right\rangle}^{x^{\prime}}\right)+\left(\mu_{\left|0^{A} 0^{B}\right\rangle}^{y^{\prime}}+\mu_{\left|1^{A} 1^{B}\right\rangle}^{y^{\prime}}\right)=0$

which complies with the spin conservation and where,

$$
\begin{aligned}
& \mu_{\left|0^{A} 0^{B}\right\rangle}=\mu_{\left|0^{A} 0^{B}\right\rangle}^{x^{\prime}}+\mu_{\left|0^{A} 0^{B}\right\rangle}^{y^{\prime}} \\
& \mu_{\left|1^{A} 1^{B}\right\rangle}=\mu_{\left|1^{A^{1} B}\right\rangle}^{x^{x^{\prime}}}+\mu_{\left|1^{1^{A} 1^{B}}\right\rangle}^{y^{\prime}}
\end{aligned}
$$

with, 


$$
\begin{aligned}
& \mu_{\left|0^{A} 0^{B}\right\rangle}^{x^{\prime}}=\mu_{\left|1^{A} 1^{B}\right\rangle}^{x^{\prime}}=0 \\
& \mu_{\left|0^{A} 0^{B}\right\rangle}^{y^{\prime}}=-\mu_{\left|1^{A^{1} B^{B}}\right\rangle}^{y^{\prime}}
\end{aligned}
$$

The second line of the equation (5.15), in relation to the original axes $\left(x^{\prime}, y^{\prime}\right)$, is the unique projection which records a perceptible value for the entanglement. That is, they are equal in modulus, parallel, opposites, and in principle, nonzero. Then, considering the equalities of equation (5.15), we will have,

$$
\begin{aligned}
& {\left[\mu_{\left|\Uparrow^{A} \Uparrow^{B}\right\rangle}, \mu_{\mid \Downarrow A \Downarrow B}\right]=\frac{\mu_{\left|0^{A} 0^{B}\right\rangle}\left(-\mu_{\left|1^{A} 1^{B}\right\rangle}\right)}{\mu_{\left|0^{A} 0^{B}\right\rangle}+\mu_{\left|1^{A} 1^{B}\right\rangle}}=\frac{\left(\mu_{\left|0^{A} 0^{B}\right\rangle}^{x^{\prime}}+\mu_{\left|0^{A} 0^{B}\right\rangle}^{y^{\prime}}\right)\left(-\left(\mu_{\left|1^{A} 1^{B}\right\rangle}^{x^{\prime}}+\mu_{\left|1^{1} 1^{B}\right\rangle}^{y^{\prime}}\right)\right)}{\left(\mu_{\left|0^{A} 0^{B}\right\rangle}^{x^{\prime}}+\mu_{\left|0^{A} 0^{B}\right\rangle}^{y^{\prime}}\right)+\left(\mu_{\left|1^{A} 1^{B}\right\rangle}^{x^{\prime}}+\mu_{\left|1^{A} 1^{B}\right\rangle}^{y^{\prime}}\right)}} \\
& =\frac{\mu_{\left|0^{A} 0^{B}\right\rangle}^{y^{\prime}}\left(-\mu_{\left|1^{A^{1} B}\right\rangle}^{y^{\prime}}\right)}{\left.\mu_{\left|0^{A} 0^{B}\right\rangle}^{y^{\prime}}+\mu_{\left|1^{1^{A} B}\right\rangle}^{y^{\prime}}\right\rangle}=\frac{\left(\mu_{\left|0^{A} 0^{B}\right\rangle}^{y^{\prime}}\right)^{2}}{0}= \pm \infty
\end{aligned}
$$

While for the new pair of axes $(x, y)$, the sum of spins will be,

$$
\mu_{\left|0^{A} 0^{B}\right\rangle}+\mu_{\left|1^{A} 1^{B}\right\rangle}=\left(\mu_{\left|0^{A} 0^{B}\right\rangle}^{x}+\mu_{\left|0^{A} 0^{B}\right\rangle}^{y}\right)+\left(\mu_{\left|1^{A} 1^{B}\right\rangle}^{x}+\mu_{\left|1^{A} 1^{B}\right\rangle}^{y}\right)=\left(\mu_{\left|0^{A} 0^{B}\right\rangle}^{x}+\mu_{\left|1^{A} 1^{B}\right\rangle}^{x}\right)+\left(\mu_{\left|0^{A} 0^{B}\right\rangle}^{y}+\mu_{\left|1^{A} 1^{B}\right\rangle}^{y}\right)=0
$$

which also complies with the spin conservation and where,

$$
\begin{aligned}
& \mu_{\left|0^{A} 0^{B}\right\rangle}=\mu_{\left|0^{A} 0^{B}\right\rangle}^{x}+\mu_{\left|0^{A} 0^{B}\right\rangle}^{y} \\
& \mu_{\left|1^{A} 1^{B}\right\rangle}=\mu_{\left|1^{A} 1^{B}\right\rangle}^{x}+\mu_{\left|1^{A} 1^{B}\right\rangle}^{y}
\end{aligned}
$$

with,

$$
\begin{aligned}
& \mu_{\left|0^{A} 0^{B}\right\rangle}^{x}=-\mu_{\left|1^{1} 1^{B}\right\rangle}^{x} \\
& \mu_{\left|0^{A} 0^{B}\right\rangle}^{y}=-\mu_{\left|1^{1} 1^{B}\right\rangle}^{y}
\end{aligned}
$$

Both lines of the equation (5.19), in relation to the new axes $(x, y)$, record a perceptible projection for the entanglement. That is, in both cases, they are equal in modulus, parallel, opposites, and in principle, nonzero. Then, considering the equalities of equation (5.19), we will have,

$$
\begin{aligned}
& {\left[\mu_{\left|\Uparrow^{A} \Uparrow^{B}\right\rangle}, \mu_{\left|\Downarrow^{A} \Downarrow^{B}\right\rangle}\right]=\frac{\mu_{\left|0^{A} 0^{B}\right\rangle}\left(-\mu_{\left|1^{A^{B}} 1^{B}\right\rangle}\right)}{\mu_{\left|0^{A} 0^{B}\right\rangle}+\mu_{\left|1^{A} 1^{B}\right\rangle}}=\frac{\left(\mu_{\left|0^{A} 0^{B}\right\rangle}^{x}+\mu_{\left|0^{A} 0^{B}\right\rangle}^{y}\right)\left(-\left(\mu_{\left|1^{A} 1^{B}\right\rangle}^{x}+\mu_{\left|1^{1^{B} 1^{B}}\right\rangle}^{y}\right)\right)}{\left(\mu_{\left|0^{A} 0^{B}\right\rangle}^{x}+\mu_{\left|0^{A} 0^{B}\right\rangle}^{y}\right)+\left(\mu_{\left|1^{A} 1^{B}\right\rangle}^{x}+\mu_{\left|1^{A} 1^{B}\right\rangle}^{y}\right)}} \\
& =\frac{\left(\mu_{\left|0^{A} 0^{B}\right\rangle}^{x}+\mu_{\left|0^{A} 0^{B}\right\rangle}^{y}\right)\left(\mu_{\left|0^{A} 0^{B}\right\rangle}^{x}+\mu_{\left|0^{A} 0^{B}\right\rangle}^{y}\right)}{\left(\mu_{\left|0^{A} 0^{B}\right\rangle}^{x}+\mu_{\left|1^{A} 1^{B}\right\rangle}^{x}\right)+\left(\mu_{\left|0^{A} 0^{B}\right\rangle}^{y}+\mu_{\left|1^{A} 1^{B}\right\rangle}^{y}\right)}=\frac{\left(\mu_{\left|0^{A} 0^{B}\right\rangle}^{x}+\mu_{\left|0^{A} 0^{B}\right\rangle}^{y}\right)^{2}}{0}= \pm \infty
\end{aligned}
$$

This shows us several things at once since the $\mathrm{W}$ and GHZ states configuration admits that: 
- the multidimensional entanglement [143] exists, as long as, the second-degree-equivalent spins are on the same plane, parallel with equal modulus, and have opposite orientation,

- the parallel operator can be applied individually: projection by projection provided that each and every one of the projections complies with

$$
\begin{aligned}
& \mu_{\left|0^{A} 0^{B}\right\rangle}^{x}+\mu_{\left|1^{A B^{B}}\right\rangle}^{x}=0 \\
& {\left[\mu_{\left|\Uparrow^{A} \Uparrow^{B}\right\rangle}^{x}, \mu_{\left|\Downarrow^{A} \Downarrow^{B}\right\rangle}^{x}\right]=\frac{\mu_{\left|0^{A} 0^{B}\right\rangle}^{x}\left(-\mu_{\left|1^{A} 1^{B}\right\rangle}^{x}\right)}{\mu_{\left|0^{A} 0^{B}\right\rangle}^{x}+\mu_{\left|1^{A} 1^{B}\right\rangle}^{x}}=\frac{\left(\mu_{\left|0^{A} 0^{B}\right\rangle}^{x}\right)^{2}}{0}= \pm \infty}
\end{aligned}
$$

and

$$
\begin{aligned}
& \mu_{\left|0^{A} 0^{B}\right\rangle}^{y}+\mu_{\left|1^{A} 1^{B}\right\rangle}^{y}=0 \\
& {\left[\mu_{\left|\Uparrow^{A} \Uparrow^{B}\right\rangle}^{y}, \mu_{\left|\Downarrow^{A} \Downarrow^{B}\right\rangle}^{y}\right]=\frac{\mu_{\left|0^{A} 0^{B}\right\rangle}^{y}\left(-\mu_{\left|1^{A^{A} 1^{B}}\right\rangle}^{y}\right)}{\mu_{\left|0^{A} 0^{B}\right\rangle}^{y}+\mu_{\left|1^{A} 1^{B}\right\rangle}^{y}}=\frac{\left(\mu_{\left|0^{A} 0^{B}\right\rangle}^{y}\right)^{2}}{0}= \pm \infty}
\end{aligned}
$$

- the application to $K$-partite of $N$ dimensional entanglement as a natural extension of equations (5.21) and (5.22) can be easily deduced by considering these equations and $§ 5.2$.

In terms of applications, the orientation is also critical since in all equations of this work despite having implicitly assumed an analysis strictly on a single plane, we have dispensed with the analysis of the projections on the three spatial axes. Besides, being the analysis performed totally generalizable to $N$ dimensions individually for each dimension, a question automatically arises: can entanglement occur independently in one direction (projection) and not in another as indicated in Figure 5.5?

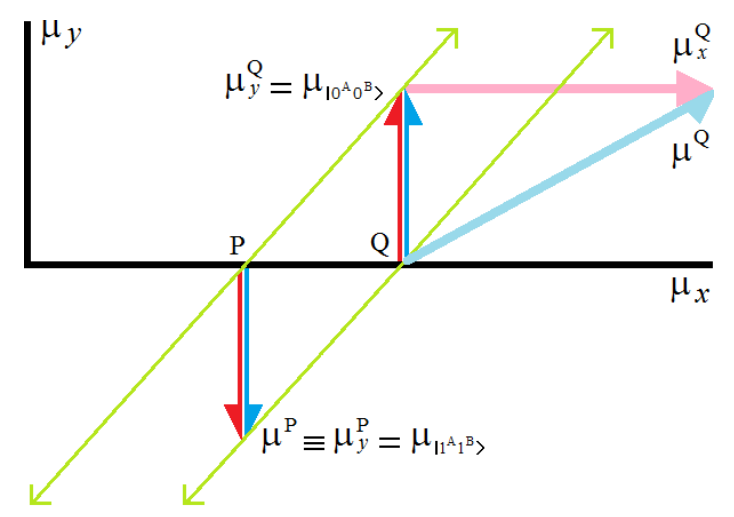

Figure 5.5 Oriented entanglement or entanglement by projection.

At first sight, $\mu^{Q}$ (in light blue) and $\mu^{P}$ (in red-blue) cannot entangle because they have different orientations and values in their spins. However, the new analysis indicates that we can consider the projections individually and thus establish an entanglement between $\mu^{P} \equiv \mu_{y}^{P}$ (in red-blue) and $\mu_{y}^{Q}$ (in red-blue), discarding the $x$ axis completely and any eventual projection on it as $\mu_{x}^{Q}$. If this is verified experimentally, we would be in the presence of an oriented entanglement or entanglement by 
projection with all the applications that this would allow in practice, e.g., a new type of radar (quantum radar [144]), new and efficient tools for quantum signal processing [145, 146], or better yet, entanglement between particles that have never coexisted spatially, among many others. The analysis based on avatars admits what has been said before, however, what about practical reality? This option is within the sphere of possibilities that this new theory contemplates as it did with the cases of $\S 3.5$. All this should not surprise us because there are other forms of partially entangled pairs [59, 60, 147, 148].

\subsection{Decoherence in the avatars' world}

The W and GHZ state configurations have their counterpart in electrical circuits, in particular, the socalled reactive circuits where they are composed, at first, by inductors (L) and capacitors (C), where the parallel operator is also used assiduously which will lead to the so-called entanglement circuits. This should not be surprising since Physics is frequently based on reusable models. In fact, the parallel operator is repeated in several cases inside Physics, e.g., we must remember equation (4.2) of $\$ 4.1$ for the De Broglie wavelength as the centre-of-mass frame of a nuclei with two masses in the context of an energy treatment where $m_{e q}=m_{1} / / m_{2}$, see pp.136 of [5]. Another example in the use of this operator can be seen in optics: if two lenses with focal length $f_{1}$ and $f_{2}$ placed next to each other are equivalent to a single lens with a focal length $f_{e q}=f_{1} / / f_{2}$, see pp.30 of [76]. Finally, the first-degreeequivalent spins (avatars) in the W and GHZ states configurations like equation (3.38) is also represented in RLC circuits [72, 73]. On the other hand, in this section and with the same criterion of §5.2, we are going to consider $X_{L}=\mu_{\left|0^{A} 0^{B}\right\rangle}$ and $X_{C}=\mu_{\left|1^{A} 1^{B}\right\rangle}$, which in the case of photons, the pair of equalities are completely equivalent. In fact, if an inductive impedance is now associated with a spin up for a given frequency, we will have,

$$
X_{L}=j \omega L=\mu_{L}
$$

From now on, $j$ only indicates a common axis of polarization, while the capacitive impedance will be,

$$
X_{C}=\frac{-j}{\omega C}=\mu_{C}
$$

then, the serial impedance $Z_{\left|\Rightarrow^{A} \Rightarrow^{B}\right\rangle}$ is -in itself- the principle of spin conservation, and its subscript $\left|\Rightarrow^{A} \Rightarrow^{B}\right\rangle$ used in its notation is purely symbolic to represent such serial configuration, being this the left graphic of Figure 5.6,

$$
Z_{\left|\Rightarrow^{A} \Rightarrow^{B}\right\rangle}=X_{L}+X_{C}=j \omega L+\frac{-j}{\omega C}=j\left(\omega L-\frac{1}{\omega C}\right)=\mu_{\left|\Rightarrow^{A} \Rightarrow^{B}\right\rangle}=\mu_{L}+\mu_{C}=0,
$$
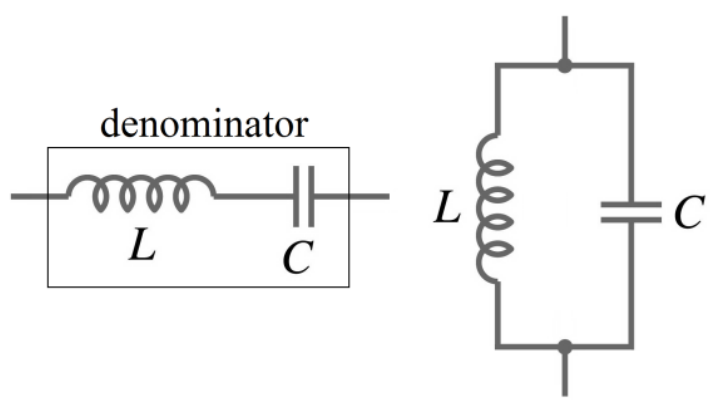

Figure 5.6 On the left, we have a serial LC circuit which is the denominator of equation (5.27), and on the right we have a parallel LC circuit.

Now, based on equation (5.25) for the resonance frequency, we will have, 
$\omega L=\frac{1}{\omega C}, \quad \omega^{2}=\frac{1}{L C}, \quad \omega=\frac{1}{\sqrt{L C}}, \quad \mathrm{f}=\frac{1}{2 \pi \sqrt{L C}}$

Besides, if we want to calculate the parallel impedance, this will be the second-degree-equivalent spin for entanglement, being this the right graphic of Figure 5.6,

$$
\left[Z_{\left|\Uparrow^{A} \Uparrow^{B}\right\rangle}, Z_{\left|\Downarrow^{A} \Downarrow^{B}\right\rangle}\right]=\frac{X_{L}\left(-X_{C}\right)}{X_{L}+X_{C}}=\frac{j \omega L\left(-\left(-\frac{j}{\omega C}\right)\right)}{j \omega L+\left(-\frac{j}{\omega C}\right)}=\left[\mu_{\left|\Uparrow^{A} \Uparrow^{B}\right\rangle}, \mu_{\left|\Downarrow^{A} \Downarrow^{B}\right\rangle}\right]=\frac{\mu_{L}\left(-\mu_{C}\right)}{\mu_{L}+\mu_{C}}= \pm \infty
$$

As we can see, equation (5.25) is similar to denominator of equation (5.2), whereas equation (5.27) is similar to equation (5.2). In Figure 5.6, we can see equation (5.25) on the left as a serial LC circuit which is the denominator of equation (5.27), while the complete version of equation (5.27) is on the right as a parallel LC circuit. This analogy resists even its application to the case of completely independent subsystems of equation (3.8), with

$$
Z_{\left|\Rightarrow^{A} \Rightarrow^{B}\right\rangle}=X_{L}+X_{L}=j \omega L+j \omega L=2(j \omega L)=\mu_{\left|\Rightarrow^{A} \Rightarrow^{B}\right\rangle}=\mu_{L}+\mu_{L}=2 \mu_{L} \neq 0,
$$

where equation (5.28) is the denominator of equation (5.29) and can be seen in the graphic on the left of Figure 5.7.

$$
Z_{\left|\Uparrow^{A} \Uparrow^{B}\right\rangle}=\frac{j \omega L j \omega L}{j \omega L+j \omega L}=\frac{(j \omega L)^{2}}{2 j \omega L}=\frac{j \omega L}{2}=\mu_{\left|\Uparrow^{A} \Uparrow^{B}\right\rangle}=\frac{\mu_{L} \mu_{L}}{\mu_{L}+\mu_{L}}=\frac{\mu_{L}}{2}<<\infty .
$$

Equation (5.29) can be seen in the graphic on the right of Figure 5.7.
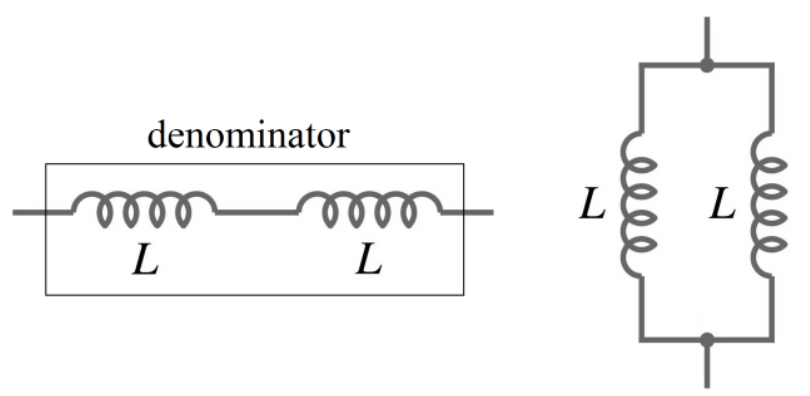

Figure 5.7 An inductive version of completely independent subsystems.

The capacitive version of Figure 5.7 can be seen in Figure 5.8.
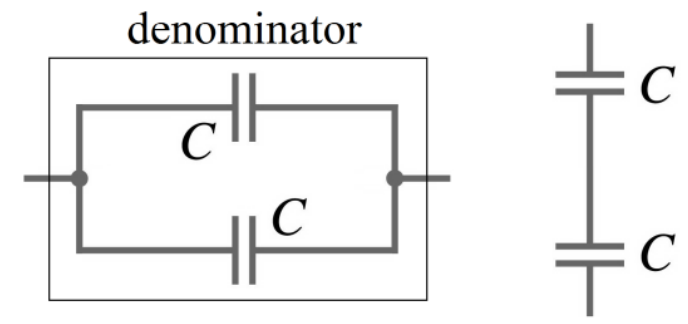

Figure 5.8 A capacitive version of completely independent subsystems. 


$$
Z_{\left|\Rightarrow^{A} \Rightarrow^{B}\right\rangle}=X_{C}+X_{C}=2 X_{C}=2\left(\frac{-j}{\omega C}\right)=\mu_{\left|\Rightarrow^{A} \Rightarrow^{B}\right\rangle}=\mu_{C}+\mu_{C}=2 \mu_{C} \neq 0,
$$

where equation (5.30) is the denominator of equation (5.31). Its graphic can be seen on the left of Figure 5.8.

$$
Z_{\left|\pi^{A} \pi^{B}\right\rangle}=\frac{X_{C} X_{C}}{X_{C}+X_{C}}=\frac{\left(X_{C}\right)^{2}}{2 X_{C}}=\frac{X_{C}}{2}=\frac{1}{2}\left(\frac{-j}{\omega C}\right)=\mu_{\left|\Uparrow^{A} \pi^{B}\right\rangle}=\frac{\mu_{C} \mu_{C}}{\mu_{C}+\mu_{C}}=\frac{\mu_{C}}{2}<<\infty .
$$

Equation (5.31) can be seen on the right of Figure 5.8.

On the other hand, the teratological case of Figure 3.14 of $\$ 3.5$ represents a W states configuration between the result of two completely independent particles that is entangled with another third particle. This example is showed in Figure 5.9 as an LC circuit. In this case, if the result of $X_{\mathrm{L}} / / \mathrm{X}_{\mathrm{L}}=$ $X_{L} / 2$ is equal and opposite to $X_{C}$, i.e., $X_{L} / 2+X_{C}=0$, then, we will have entanglement, since the denominator of parallel operator must be zero so that the result is infinite. In other words, this last equation is the denominator of parallel operator of equation (5.32) and it is on the left of Figure 5.9, while on the right of Figure 5.9 we will have the parallel operator of equation (5.32),

$$
\left[Z_{\left|\pi^{A} \pi^{B}\right\rangle}, Z_{\left|\Downarrow^{A} \Downarrow^{B}\right\rangle}\right]=\frac{X_{L} / 2\left(-X_{C}\right)}{X_{L} / 2+X_{C}}=\frac{\frac{j \omega L}{2}\left(-\left(-\frac{j}{\omega C}\right)\right)}{\frac{j \omega L}{2}+\left(-\frac{j}{\omega C}\right)}=\left[\mu_{\left|\pi^{A} \Uparrow^{B}\right\rangle}, \mu_{\left|\Downarrow^{A} \Downarrow^{B}\right\rangle}\right]=\frac{\mu_{L} / 2\left(-\mu_{C}\right)}{\mu_{L} / 2+\mu_{C}}= \pm \infty
$$

We must remember that, originally, this teratological case does not comply with the conservation of the spin, since $X_{C}+X_{L}+X_{L} \neq 0$, however, $X_{C}+X_{L} / 2=0$ does.
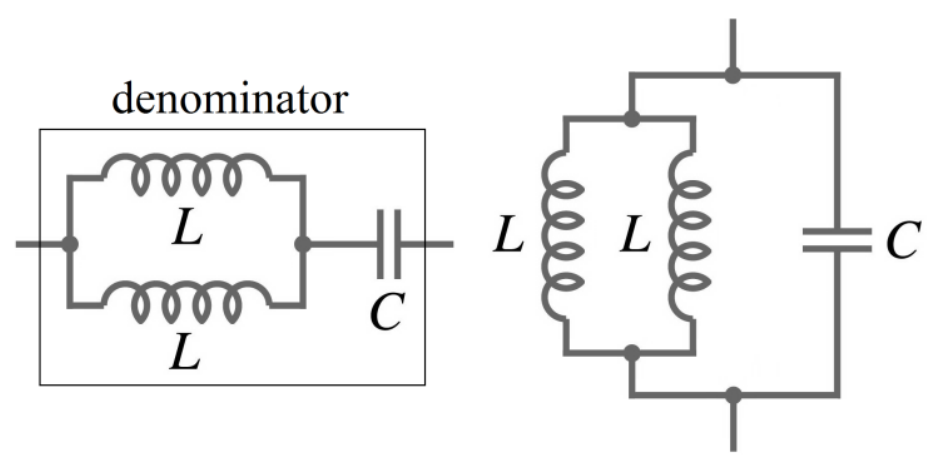

Figure 5.9 Two completely independent particles are entangled with another third particle.

Including the most extreme cases, this analogy between spins and LC circuits fits the W and GHZ state configurations, even under the effects of quantum measurement [22], however a question arises: what about the collapse of the wave-function? It is a very interesting question, in fact, this analogy based on LC circuits for $\mathrm{W}$ and GHZ state configurations resist the presence of decoherence. It is important to highlight that we can continue to use this analogy to represent the disturbed effect of entanglement intervened by the action of the environment since the scope is not adiabatic. The presence of decoherence is modelled via resistors in an RLC analogy in Figure 5.10, which represents both the degradation of the equivalent inductor and the capacitor simultaneously. In other words, and in the same way that happens in electrical and electronic circuits, the presence of resistors degrades both the inductor and the capacitor leading us to present a theorem on decoherence based on the RLC analogy and its corresponding proof. 

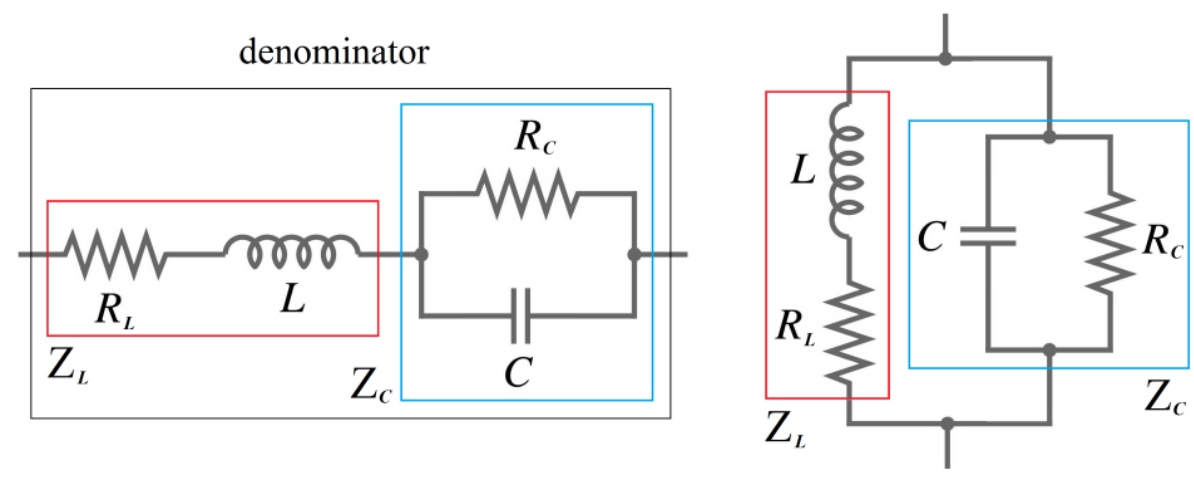

Figure 5.10 Entanglement perturbated by decoherence represented with resistors.

Theorem: Decoherence converts two entangled spins into two new equivalent and completely independent spins.

Proof: First, we are going to simplify the RC tank circuit of Figure 5.10, which is framed in blue.

$$
\begin{aligned}
Z_{C} & =\frac{R_{C} X_{C}}{R_{C}+X_{C}}=\frac{R_{C}\left(\frac{-j}{\omega C}\right)}{R_{C}-\frac{j}{\omega C}}=\frac{R_{C}\left(\frac{-j}{\omega C}\right)\left(R_{C}+\frac{j}{\omega C}\right)}{\left(R_{C}-\frac{j}{\omega C}\right)\left(R_{C}+\frac{j}{\omega C}\right)}=\frac{R_{C}^{2}\left(\frac{-j}{\omega C}\right)+R_{C}\left(\frac{1}{\omega C}\right)^{2}}{R_{C}^{2}+\left(\frac{1}{\omega C}\right)^{2}} \\
& =\frac{R_{C}\left(\frac{1}{\omega C}\right)^{2}-j R_{C}^{2} \omega C\left(\frac{1}{\omega C}\right)^{2}}{R_{C}^{2}+\left(\frac{1}{\omega C}\right)^{2}}=\frac{R_{C}+\left(-j R_{C}^{2} \omega C\right)}{1+\left(\omega C R_{C}\right)^{2}}=\frac{R_{C}}{1+\left(\omega C R_{C}\right)^{2}}+\frac{\left(-j R_{C}^{2} \omega C\right)}{1+\left(\omega C R_{C}\right)^{2}} \\
& =R_{C}^{\prime}+\left(\frac{-j}{\omega C^{\prime}}\right)
\end{aligned}
$$

where,

$$
R_{C}^{\prime}=\frac{R_{C}}{1+\left(\omega C R_{C}\right)^{2}}
$$

and,

$$
\frac{1}{\omega C^{\prime}}=\frac{R_{C}^{2} \omega C}{1+\left(\omega C R_{C}\right)^{2}}, \quad \omega C^{\prime}=\frac{1+\left(\omega C R_{C}\right)^{2}}{R_{C}^{2} \omega C}, \quad C^{\prime}=\frac{1+\left(\omega C R_{C}\right)^{2}}{R_{C}^{2} \omega^{2} C}
$$

Then, we will go from Figure 5.10 to Figure 5.11. In consequence, the complete impedance of the graphic on the right of Figure 5.11 will be,

$$
Z_{\left|\Uparrow^{A} \Uparrow^{B}\right\rangle}=\frac{Z_{L} Z_{C}}{Z_{L}+Z_{C}}=\frac{\left(R_{L}+X_{L}\right)\left(R_{C}^{\prime}+X_{C}^{\prime}\right)}{\left(R_{L}+X_{L}\right)+\left(R_{C}^{\prime}+X_{C}^{\prime}\right)},
$$

where its denominator will be, 

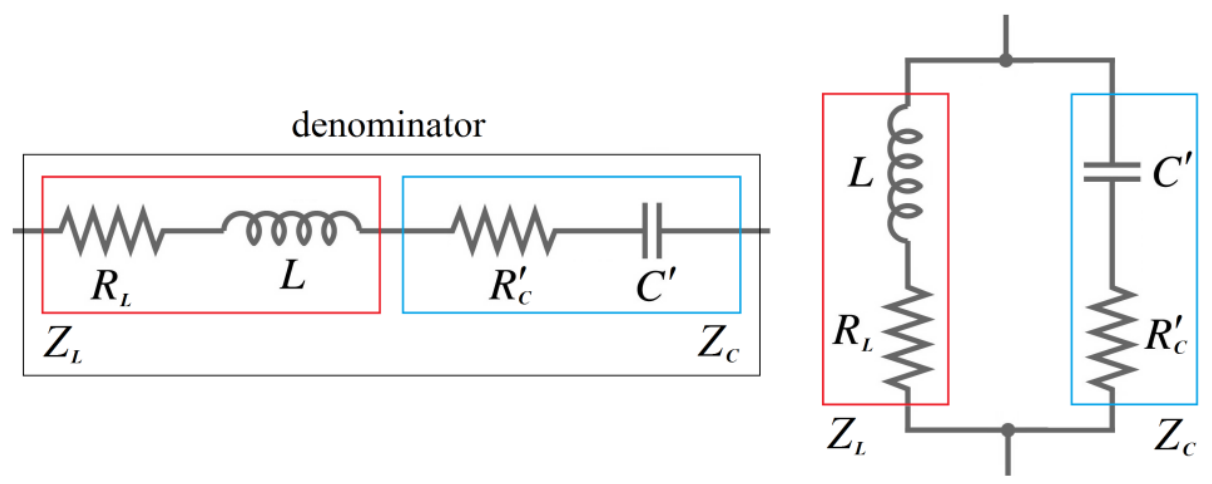

Figure 5.11 RLC circuit of Figure 5.10 with a built-in simplification on the original RC circuit.

$$
\begin{aligned}
Z_{\left|\Rightarrow^{A} \Rightarrow^{B}\right\rangle} & =Z_{L}+Z_{C}=\left(R_{L}+X_{L}\right)+\left(R_{C}^{\prime}+X_{C}^{\prime}\right)=\left(R_{L}+R_{C}^{\prime}\right)+\left(X_{L}+X_{C}^{\prime}\right) \\
& =\left(R_{L}+\frac{R_{C}}{1+\left(\omega C R_{C}\right)^{2}}\right)+\left(j \omega L+\frac{\left(-j R_{C}^{2} \omega C\right)}{1+\left(\omega C R_{C}\right)^{2}}\right) \\
& =\left(R_{L}+\frac{R_{C}}{1+\left(\omega C R_{C}\right)^{2}}\right)+j \omega\left(L-\frac{R_{C}^{2} C}{1+\left(\omega C R_{C}\right)^{2}}\right) \neq 0
\end{aligned}
$$

The imaginary part of equation (5.37) may be canceled for some frequency, however, its real part will never be annulled, as we can see in Figure 5.12 where $Z_{L}$ is in pink while $Z_{C}$ is in light blue and both of which shape $Z_{\left|\Rightarrow^{A} \Rightarrow^{B}\right\rangle}$ in light green which will never be annulled. Therefore, we find ourselves facing a new 2-partite equivalent system of completely independent spins.

Note: this theorem, as well as its proof, is easily and completely generalizable to the $N$-partite case.

Therefore and undoubtedly, for this analogy, the resistors represent decoherence in the same way that they are associated with the energy expenditure by Joule's effect in electrical circuits [73]. Since in this analogy energy is lost in heat, the resistors will represent the loss of entanglement because of the interaction with the environment. If we consider that the ambit where the entangled particles are located is not adiabatic, they will be victims of the action of decoherence [22]. So far decoherence seems like the bad boy of the movie, however, it is an excellent tool to understand this analogy better.

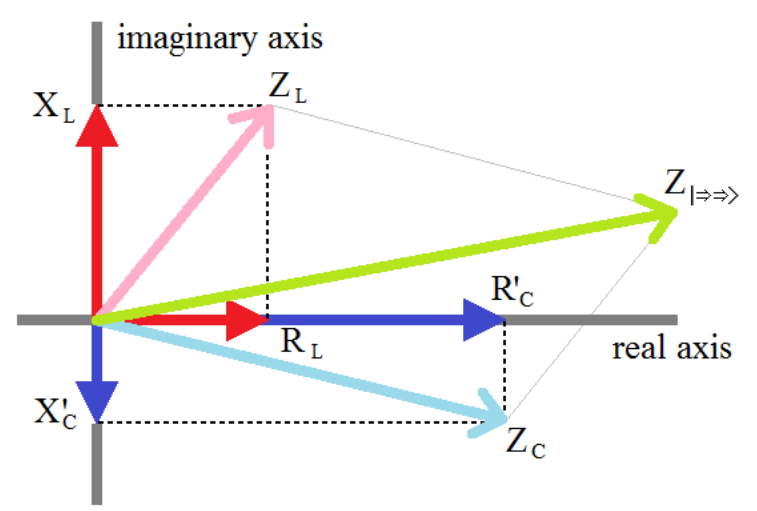

Figure 5.12 The denominator of equation (5.36), which is the graphic on the left of Figure 5.11. 
Finally, from equation (5.27)

$$
\left[Z_{\left|\Uparrow^{A} \Uparrow^{B}\right\rangle}, Z_{\left|\Downarrow^{A} \Downarrow^{B}\right\rangle}\right]=\frac{j \omega L}{1-\omega^{2} L C}=\left[X_{L} \gamma^{2}, X_{C} \gamma^{2}\right] \equiv\left[\mu_{\left|\Uparrow^{A} \Uparrow^{B}\right\rangle}, \mu_{\left|\Downarrow^{A} \Downarrow^{B}\right\rangle}\right]=\left[\mu_{\left|0^{A} 0^{B}\right\rangle} \gamma^{2}, \mu_{1^{\left.1_{1} 1^{B}\right\rangle}} \gamma^{2}\right]
$$

where $\gamma$ is the Lorentz's factor,

$$
\gamma=\frac{1}{\sqrt{1-\omega^{2} L C}} .
$$

Then, regardless of our axis reference $j$, the avatars will be

$$
\begin{aligned}
& \left(Z_{\left|\Uparrow^{A}\right\rangle}=\sqrt{\left|X_{L}\right|} \gamma\right) \equiv\left(\mu_{\left|\Uparrow^{A}\right\rangle}=\mu_{\left|0^{A}\right\rangle} \gamma=+\infty\right) \\
& \left(Z_{\left|\Downarrow^{A}\right\rangle}=-\sqrt{\left|X_{L}\right|} \gamma\right) \equiv\left(\mu_{\left|\Downarrow^{A}\right\rangle}=-\mu_{\left|0^{A}\right\rangle} \gamma=\mu_{\left|1^{A}\right\rangle} \gamma=-\infty\right) \\
& \left(Z_{\left|\Uparrow^{B}\right\rangle}=\sqrt{\left|X_{C}\right|} \gamma\right) \equiv\left(\mu_{\left|\Uparrow^{B}\right\rangle}=\mu_{\left|0^{B}\right\rangle} \gamma=+\infty\right) \\
& \left(Z_{\left|\Downarrow^{B}\right\rangle}=-\sqrt{\left|X_{C}\right|} \gamma\right) \equiv\left(\mu_{\left|\Downarrow^{B}\right\rangle}=-\mu_{\left|0^{B}\right\rangle} \gamma=\mu_{\left|1^{B}\right\rangle} \gamma=-\infty\right)
\end{aligned}
$$

where, we can see that equation (5.38) is equal to equation (3.38), equation (3.44) is equal to equation (5.39) and equation (5.40) is equal to equation (3.43), i.e., the models are completely equivalent and therefore this interpretation of decoherence is perfectly valid.

\subsection{Loss and restoration of entanglement}

We know that an entanglement link is established between elements of an EPR pair. Figure 5.13 represents such link as two tank-tuned-LC-circuits based on the guidelines of §5.4.

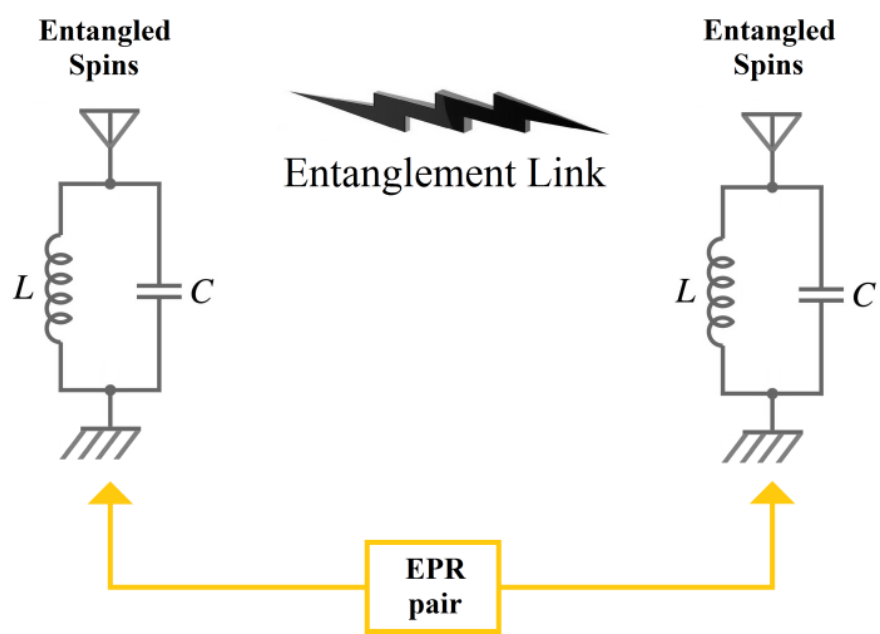

Figure 5.13 Entanglement link between an EPR pair. This pair is interpreted like two tuned circuits.

A problem arises when we want to measure the state of one of the elements of the entangled pair. In this circumstance, the entanglement is interrupted. In Figure 5.14 the intervention of the quantum measurement is represented as resistors in the LC (inductor-capacitor) pairs. These resistors tell us 
about the direct intervention of the environment on these pairs. As a result of this, the spins become completely independent and the avatars disappear, see $\$ 5.4$.
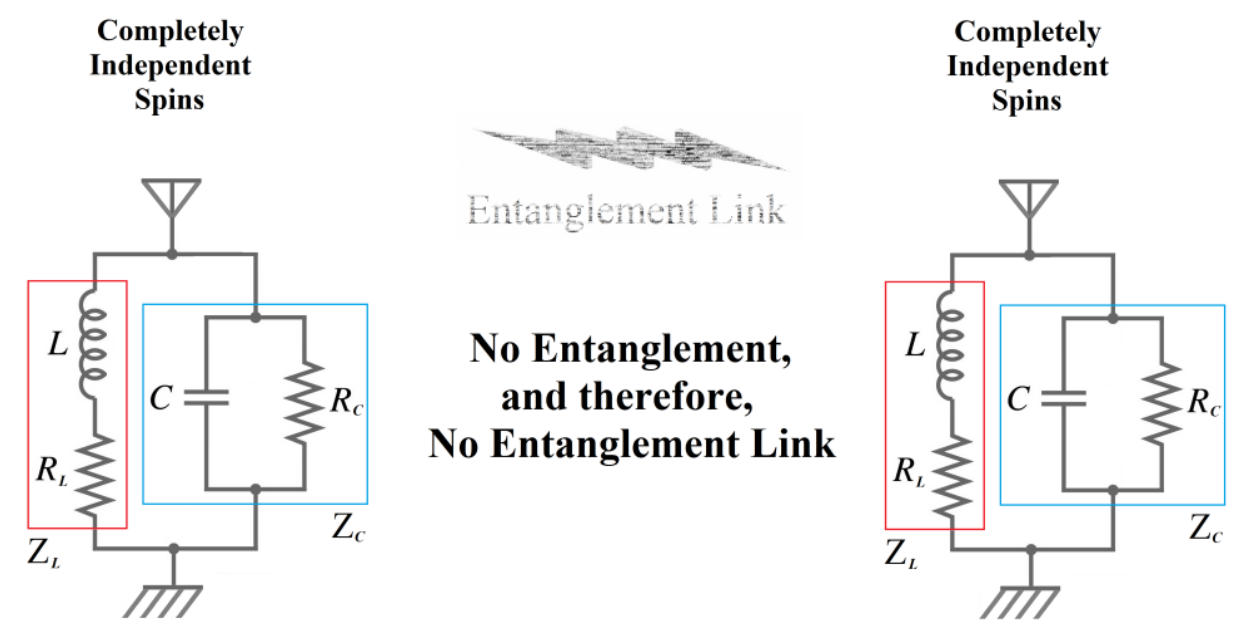

Figure 5.14 After quantum measurement the entanglement link is disrupted, however, the analysis based on avatars admits that entanglement could persist in a ghostly way as an eventual possibility. Both resulting spins will be completely independent, and the action of the decoherence is represented as a degradation of the components of the EPR pair with resistors inside inductors and capacitors.

As we can see in Figure 5.15, the resistor components (in grey) of the original entangled spins $\mu_{\left|0^{A} 0^{B}\right\rangle}$ (in red-blue) and $\mu_{\left|1^{A} 1^{B}\right\rangle}$ (in red-blue) generate two new resulting spins of second degree (in pink and in light blue, respectively) which are not entangled. Effectively, the resistive components misalign the original spins (i.e., they are not parallel), making them independent, thus, interrupting the entanglement. See $\$ 5.4$.

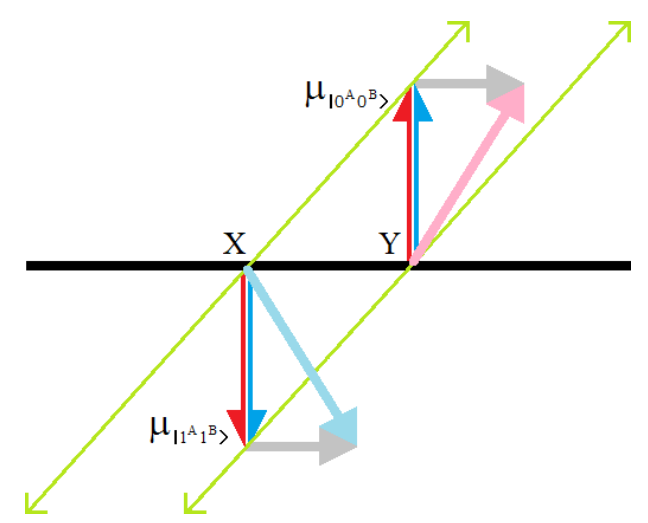

Figure 5.15 Original entangled spins $\mu_{\left|0^{A} 0^{B}\right\rangle}$ (in red-blue) and $\mu_{\left|1^{A} 1^{B}\right\rangle}$ (in red-blue) with their respective resistor components in grey, which generate two new not collinear, and hence, not entangled resulting spins (in pink and in light blue, respectively).

This is also known as decoherence and is associated with the collapse of the wave-function [149]. Besides, noise as a consequence of an interaction with the environment or with operations on imperfect gates generally reduces both purity and entanglement itself of a given state [22]. However, if one has several copies of some less than all available maximally-entangled-state, it is possible that both parties Alice and Bob concentrate or distill the entanglement [150-155] by acting locally on their parts in relation to the states and exchanging classical information on a classical channel [54-60]. Thus, by 
using the so-called local operations and classical communication (LOCC) [156] we can create fewer pairs with higher entanglement and higher degree of purity [150]. This process is called entanglement purification or entanglement distillation [1,3,51, 150-155]. So far, this was the only known technique to improve entanglement, although, it was only possibly done partially. However, the analysis of $\$ 5.4$ based on avatars tells us that the loss of entanglement could be a completely reversible process, in other words, the entanglement could be restored and the link re-established. Moreover, based on §5.4, if the EPR pairs are LC circuits, the decoherence will be circuits degraded by a resistive presence in each component and given that the resistors are directly associated with heat dissipation, the technique suggested to restore the entanglement is, obviously, the application of cold. But, what is the equivalent in the real case? In other words, what could be applied locally to each particle to restore the entanglement? Laser cooling [157, 158]? Returning to Figure 5.14, the analysis based on avatars tells us that the decoherence could be overcome with a direct and local action on the spins (may be, local cooling), although these are independent with an apparent irreversibility.

Everything said so far would be indicating that entanglement remains latent after decoherence in a ghostly state. Besides, such entanglement can resurrect with a direct and individual action on the spins as we can see in the lower part of Figure 5.16. This has never been predicted in such a direct way by Quantum Mechanics [5-9] although reversibility is the fifth essence of this theory since it is a mandatory requirement of it. However, this must still be verified experimentally.

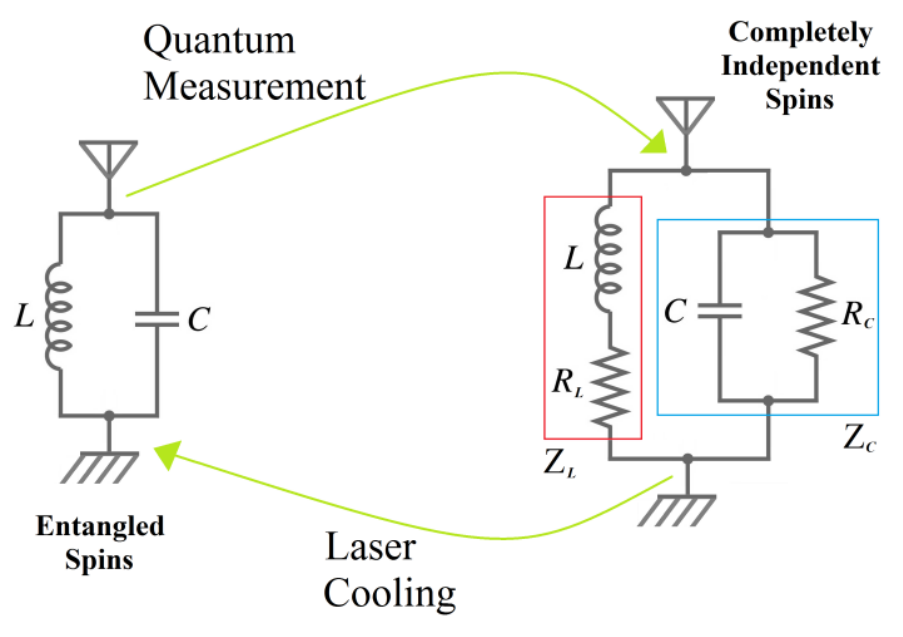

Figure 5.16 For a given EPR element, the upper green arrow shows the passage from the entangled spins to completely independent spins via quantum measurement, while, the bottom green arrow indicates the possible transition from completely independent spins to entangled spins thanks to an eventual process, may be, laser cooling. Laser cooling would eliminate the resistor factors that inhibit the entanglement.

Figure 5.17 shows the avatars of an entanglement process in the upper part, a quantum measurement and its counterpart (i.e., any eventual and possible restorative process) in the middle, and the avatars of two completely independent particles in the lower part. The aforementioned restoration process should be almost miraculous because while the avatars of two initially entangled particles are 4 and with spins infinitely superior in magnitude to that of the original particles which once subjected to a quantum measurement process, their avatars will be reduced to 2 with a smaller magnitude than the original particles. In other words, to restore the entanglement, both of the avatars should duplicate or divide themselves into two and expand their magnitude relativistically.

However, if restoration was possible, there would be two possibilities:

- two types of independent particles: per se (i.e., without any previous connection) or those that result from a quantum measurement after having been entangled: independent-after-entanglement (IAE), or

- a single type of independent particles, which could be entangled between each other although they have never coexisted spatially before. This last possibility is more difficult to digest. 


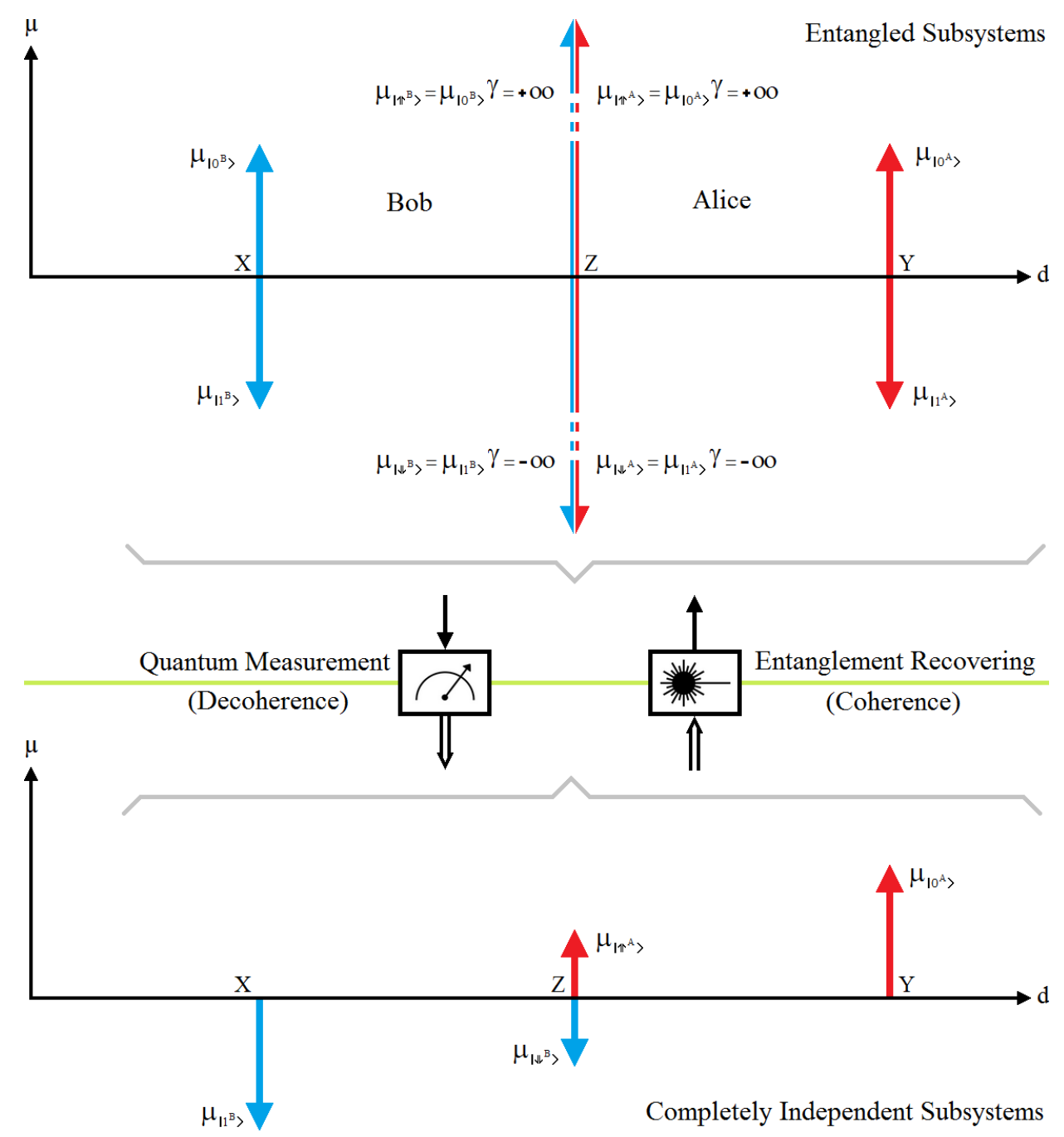

Figure 5.17 The analysis based on avatars admits a bidirectional process: from the entangled particles to the completely independent particles (via a non-coherent process like quantum measurement) and vice versa (thanks to any restorative process).

This analysis is not trivial at all since for the completely independent case we see that the equation (3.1) tells us about 4 original spins involved in the development of the deduction of avatars although, at the end, we only get 2 avatars from such development. The upper part of Figure 5.18 shows the equivalence of models for 2 completely independent particles that were previously treated in Figure 2.1. In both cases, and until a quantum measurement is carried out, there is an indeterminacy about what kind of spin Alice and Bob have, an indeterminacy which is represented by 2 original spins in the hands of them. However, the lower part of Figure 5.18 also tells us of 2 completely independent particles which arise as a result of $\S 3.1$ but are also the consequence of a quantum measurement on previously entangled particles. In other words, in all the analysis carried out from $\$ 3.1$ onwards we implicitly assume an independence that originates after a quantum measurement on a previously entangled pair. This is why, we refer to a situation like that which can be observed in the lower part of Figure 5.18, which is very different from what is shown in the upper part of said figure. This difference is remarkable since in the example of the upper part of Figure 5.18, Alice and Bob do not know if they have a spin-up or a spin-down, while in the example of the lower part there is no doubt about this. In both cases, we speak of two completely independent particles although the option in the lower part of Figure 5.18 clearly comes from a pre-existing and binding entanglement which has been a victim of decoherence. This difference would allow us to go from the bottom to the top of Figure 5.17 since in the post-measurement model there are ashes of a pre-existing entanglement and in the other one, there are not. 

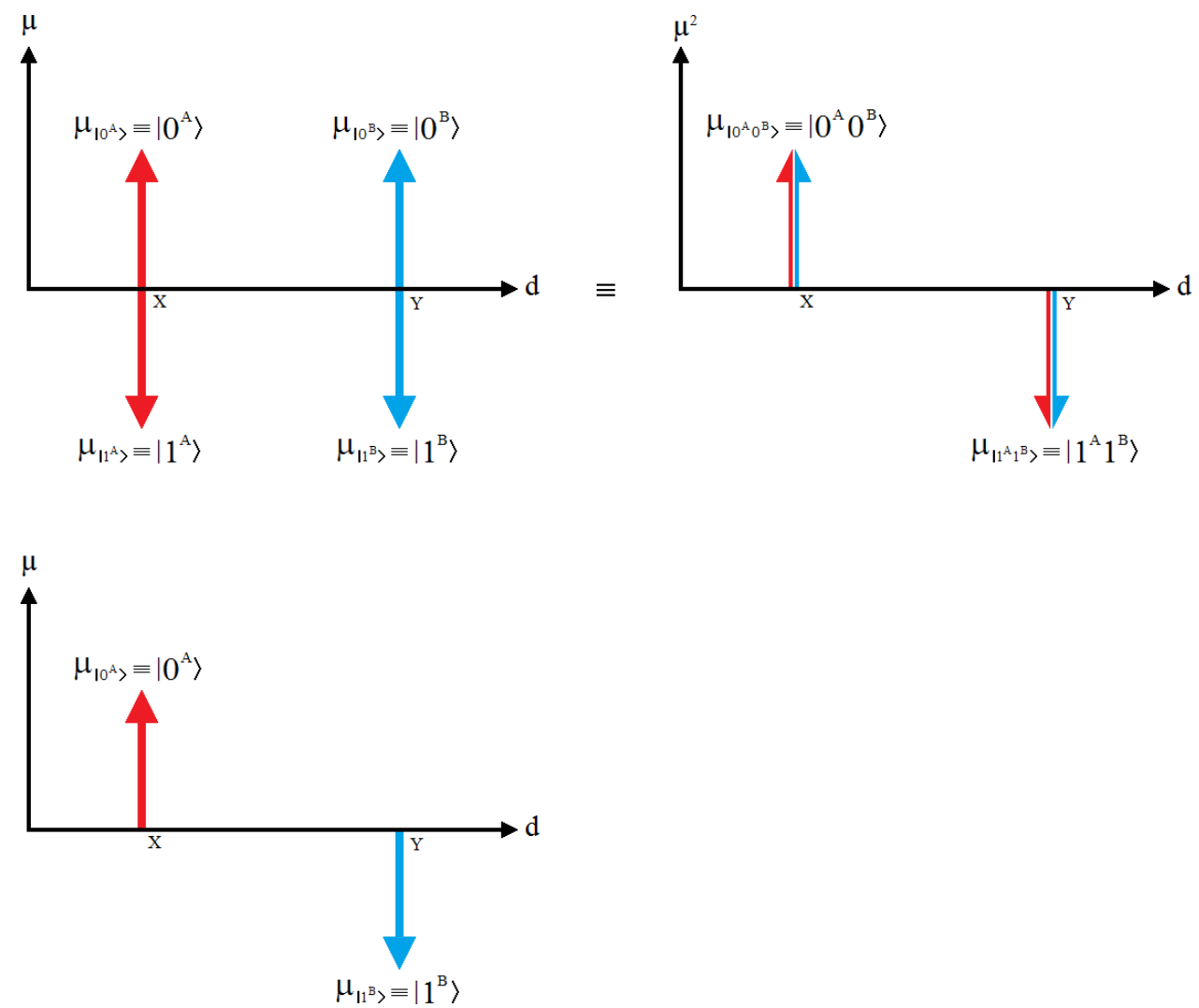

Figure 5.18 Analysis of two situations about completely independent particles: above is per se or without any previous connection, and down is independent-after-entanglement (IAE), i.e., after a quantum measurement.

\section{Consequences of the avatars' existence}

This section is undoubtedly interesting from a practical point of view since in it, we try to answer open topics of Modern Physics from the existence of the avatars. In this sense, we will explore the intervention of the avatars in several important fields of Physics in which entanglement is involved:

- wormholes resulting from an entanglement process (WREP),

- quantum communications [62-67], with a particular emphasis in quantum teleportation [54-60],

- analysis about the locality problem [37-41] with avatars,

- Bell's theorem [30] and the avatars, and

- GR = QM, quantum gravity and anti-gravity.

\subsection{Analysis of WREPs}

In this subsection, we will demonstrate that every WREP is traversable, i.e., it is equivalent to another wormhole but of null length (like a slice or ring) which can be crossed instantaneously with dramatic implications on quantum communications treated in $§ 6.2$. That equivalent wormhole (slice or ring) is also located at an intermediate point which is equidistant from the original entangled particles that have generated it and where avatars are also found. Such original entangled particles can be those resulting from, for example, the Hawking's radiation [13]. In that case, we would be talking about particle-anti-particle pairs and, as in any other case, the WREP will be open and functioning while the entanglement lasts. In other words, the avatars are to the original entangled particles what the slice or ring is to the original wormhole formed between two black holes since if the distance between avatars is zero, the equivalent wormhole length should also be zero; therefore they are equivalent, for this reason we say that the original wormhole is reduced to a ring or slice, which can be seen in the lower part of Figure 6.1. The upper part of this figure shows two completely independent elements which do not generate a wormhole. Behind the word elements, we align the particles or anti-particles necessary for the entanglement. 

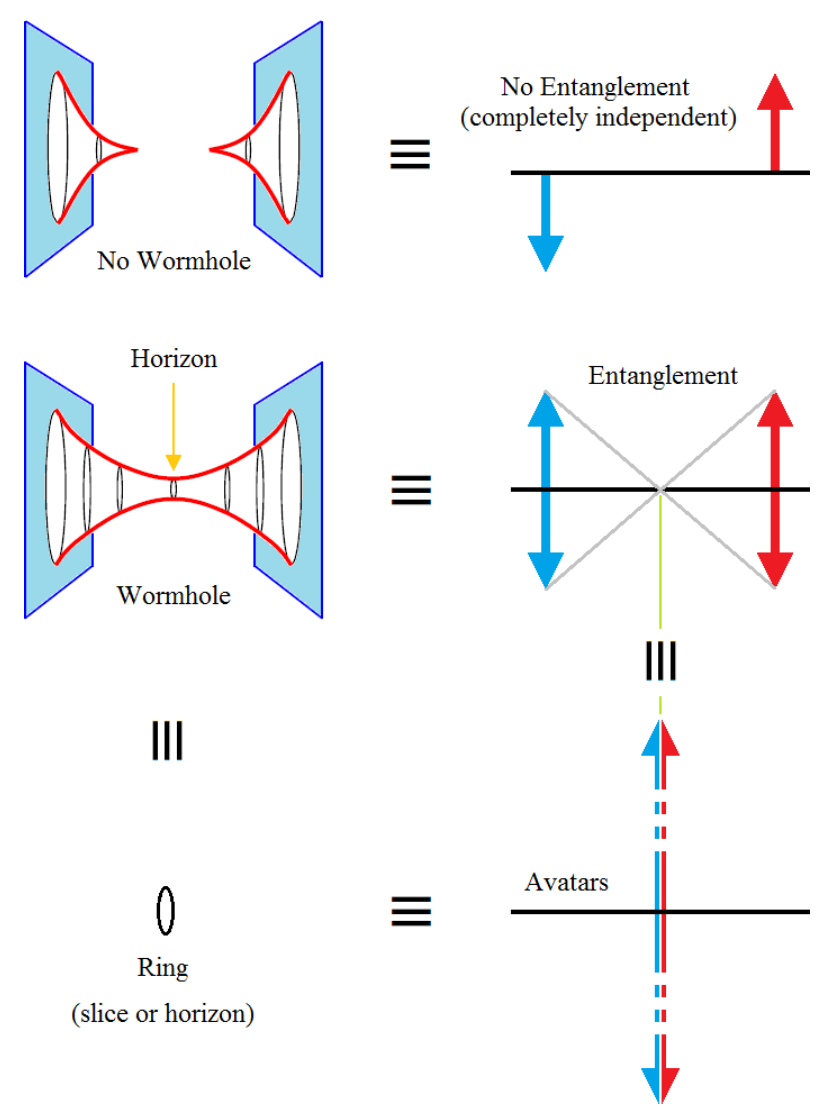

Figure 6.1 WREP is reduced to a ring of null thickness (slice or horizon).

Besides, the middle part of Figure 6.1 shows two entangled elements which are equivalent to an operative wormhole. But, the key idea is represented in the lower part of this figure since, as we saw in $\$ 3.3$, the distance between avatars is zero which indicates that there is no path, distance or channel between them. Therefore, that equivalent wormhole will have a length equal to zero, and any path of such length is perfectly traversable without any type of difficulty or energy expenditure. On the other hand, Figure 6.2 shows the result of what was said above in relation to a very pronounced curvature of space-time called hyperspace [159], where the original wormhole is a shortcut. The graph on the right of Figure 6.2 shows the complete curvature of hyperspace to the point of provoking an equivalent super-compression (or overshrinking) on the wormhole or channel which is reduced to a ring or slice.

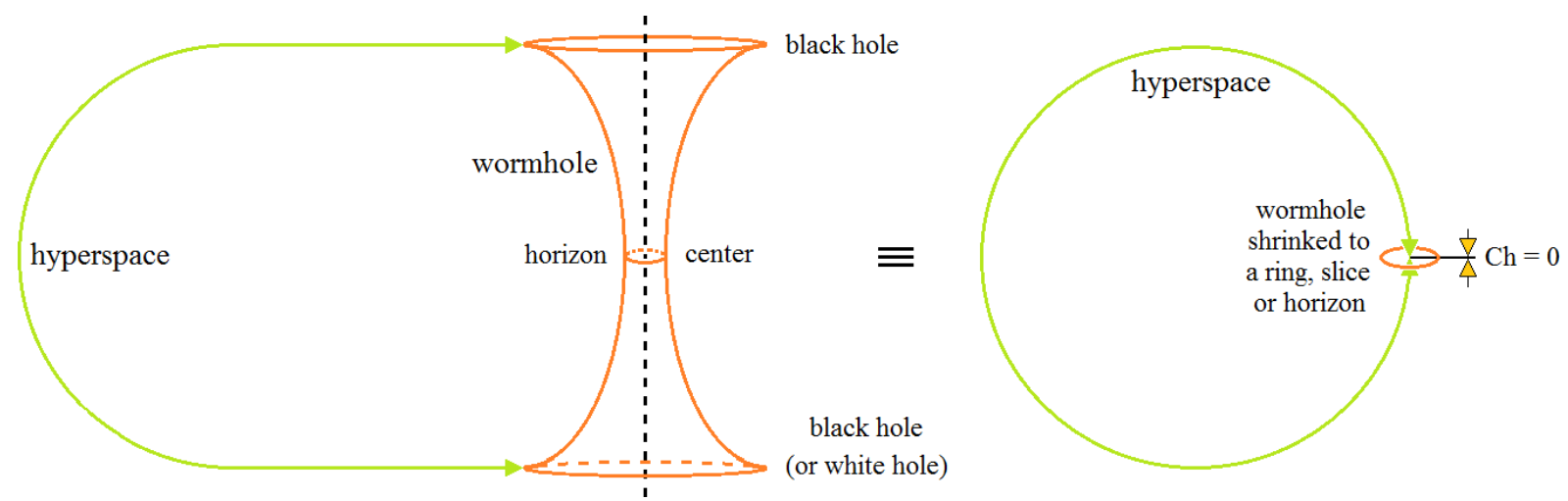

Figure 6.2 Graph on the left with a very pronounced curvature of hyperspace where the original wormhole is a shortcut. Graph on the right shows the complete curvature of hyperspace to the point of provoking a supercompression (or overshrinking) on the channel (ER bridge or wormhole). This last graph is the platform to analyze the virtues of entanglement in communications. 
Moreover, in $\S 4.2$ we saw that the avatars conserve the mass between them, reason why half of them have negative mass (negative matter) on both sides of the equivalent wormhole (ring or slice), which keeps it stable since the negative counteracts the positive ones. This fact gives stability to the original wormhole while something crosses it, that is, while the entanglement is active. It is useful to remember, in addition to $\$ 4.2$, that avatars imply infinite energies in opposition. To clarify this, let us suppose that what tries to cross the original wormhole is a turtle and considering that a WREP is in itself traversable (because it has a zero transversal length since all the avatars are together in one point only) then the turtle will travel the entire path instantaneously even if its speed is greatly lower than the speed of light. In other words, as a consequence of the avatars and their location, the channel is null, the WREP is equivalent to a ring or slice and the entanglement does not need superluminal signaling to be instantaneous. Simultaneously, as mentioned before, the avatars are the plenipotentiary ambassadors of the original spins and they are also the ones that sustain the entanglement. Since this is instantaneous, it does not contradict or conflict the postulates of the GR, therefore, QM can be said to be a complete theory able to explain this fascinating effect: entanglement. In fact, from the equation (3.43) we can see that the entanglement uses GR in the avatars, and this is done so as not to violate the postulates of the GR, or in other words, GR assists the entanglement so that it does not violate it. Besides, in its most generic form, equation (3.43) is:

$$
\mu_{|X\rangle}=\mu_{|x\rangle} \gamma
$$

where, $X$ is the subscript of the avatars, which can be $\Uparrow$ or $\Downarrow$, while, $x$ is the subscript of the original spins, respectively, which can be 0 or 1 . In this way, we arrive at a unified effect equation. This is the most faithful testimony of the relativistic nature of spins (avatars) involved in quantum entanglement. Besides, equation (6.1) tells us three important things:

- this equation is the reason why we say that the avatars are the hinge between GR and QM, which brings us closer to the GR=QM conjecture [71],

- if within the ER=EPR conjecture [17-19] we are speaking of entanglement, it is imperative to take what has been said so far, and make the necessary adjustments in it in order to:

a) consider that all WREP are traversable,

b) avoid an inadmissible contradiction between $\mathrm{GR}=\mathrm{QM}$ and $\mathrm{ER}=\mathrm{EPR}$, which are not exempt from detractors [160] and patches [161-164],

- equation (6.1) is a balcony facing the Theory of Everything (TOE) [20].

Furthermore, an equivalent version of the equation (6.1) but in Dirac's notation would be

$$
|X\rangle=|x\rangle^{\otimes \gamma}
$$

where considering for a moment that $\gamma \in \mathbb{N}$, then, $\otimes \gamma$ means $\gamma$ copies of $|x\rangle$. Evidently, this speaks to us of an infinitely large vector. Equation (6.2) implies a qubit on a Bloch's sphere of infinite radius. It is as if Bloch's sphere was also the victim of the same homothety. Besides, we wonder if it is possible to go from $|x\rangle$ to $|X\rangle$ through a unitary operator, as in the case of a quantum algorithm [51-53]

$$
|X\rangle=U|x\rangle
$$

The first problem that we detected respect to equation (6.3) is that there is a severe dimensional problem between $|X\rangle$ and $|x\rangle$, because, while $|x\rangle$ is a vector of two rows by one column, $|X\rangle$ is another vec-tor but of $\gamma$ rows by one column. Besides, $U$ is not unitary and has no inverse, therefore, in this way the quantum nature of the avatars is compromised, but their relativistic face is not. Thus, we are certain to say that the avatars are not clones of the original spins. 
On the other hand, an interesting question arises: can we make the contraction of the channel inside WREP and its consequence on the instantaneity of the entanglement explicit? As expected, in the vicinity of the avatars the space is contracted according to the homothety seen in the previous sections,

$\Delta x_{\text {avatars }}=\Delta x_{\text {originals }} / \gamma$

and being $\Delta x=c \Delta t$, then, the transit time between avatars will be,

$\Delta t_{\text {avatars }}=\Delta t_{\text {originals }} / \gamma$

$\Delta x_{\text {avatars }}$ is the space or length of the channel, in other words, the separation between avatars, while $\Delta t_{\text {avatars }}$ is the time that takes to go through that $\Delta x_{\text {avatars. }}$. As we mentioned before, the fact that we do not know exactly where the original entangled particles are, or how far apart they are do not matter, the avatars will all be together at an intermediate point equidistant from the original spins. This means that the analysis carried out in this subsection is absolutely valid and, at the same time, indifferent to the inter-spin separation existing between the original particles.

\subsection{Analysis in quantum communications}

This subsection is composed of two parts where we will analyze:

- the impact of $\S 6.1$ on an eventual entanglement for quantum communications [62-67], and

- the consequences and the implications of the research done in this paper on teleportation and superdense coding [54-60].

The first analysis begins with the condition that if the channel defined by the entanglement is equivalent to the separation between avatars, then said channel has zero length, that is, there is no channel, or that channel is reduced to a point, where all the avatars are together. Now, if there is not a channel, then, there are four direct consequences:

a) what you put at that point is equal to what you get from that point (WYPWYG), i.e., that channel has infinite bandwidth and null latency (anything that crosses it will do it instantaneously), then, in this case, we are not talking about transmitting but about sharing information in that point channel,

b) if there is no channel, then there is no channel noise, i.e., the link is perfectly robust,

c) if the bandwidth is infinite and the noise is zero, then the capacity of that channel is also infinite [61], and

d) what is more, it is impossible to attack, intercept or hack a channel that does not exist.

Other minor consequences appear since the avatars are all together at the same point, therefore, a link based entanglement does not suffer fading, vanishing, nothing interrupts it, it does not need antennas, or high power transmission systems. Also, it is important to mention that the entanglement is a monogamous process [1-3]. Summing up, a link based on entanglement is then the epitome of communications making data compression and security unnecessary.

The second analysis implies a simplified version of the quantum teleportation protocol free of disambiguation thanks to bits obtained from a Bell States Measurement process (BSM) and free of the transmission of these bits through a classic channel [56] (with all that this means in the relation between GR and QM). We must resort to everything seen so far in the present work to achieve this. We will explore the standard teleportation protocol [56], as well as the simplified version with and without noise [165]. Finally, we will analyze the performance of classical-to-quantum and quantum-to-classical interfaces thanks to which we will implement a new and simplified superdense coding scheme that only uses a link based on entanglement for the transmission of the mentioned bits.

Therefore, we will start generating the Bell's bases from the Computational Basis States (CBS), the Hadamard $(H)$ and the CNOT gates [51]. 
As an example $[1,2,51]$, let us try to get the base $\left|\beta_{00}\right\rangle$ in terms of Figure 6.3 with $|x\rangle=|y\rangle=|0\rangle$.

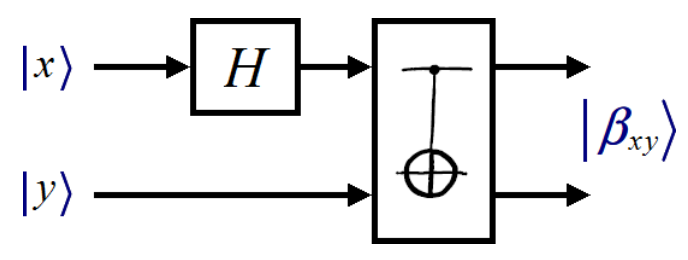

Figure 6.3 The output of these gates, Hadamard $(H)$ and CNOT, is an EPR qubit or Bell's state. In fact, an EPR pair where one EPR is for Alice and the other one for Bob.

The process begins with the Hadamard $(H)$ matrix [51]:

$I \otimes H=\frac{1}{\sqrt{2}}\left[\begin{array}{ll}1 & 0 \\ 0 & 1\end{array}\right] \otimes\left[\begin{array}{cc}1 & 1 \\ 1 & -1\end{array}\right]=\frac{1}{\sqrt{2}}\left[\begin{array}{cccc}1 & 0 & 1 & 0 \\ 0 & 1 & 0 & 1 \\ 1 & 0 & -1 & 0 \\ 0 & 1 & 0 & -1\end{array}\right]$

where $I$ is a 2-by-2 identity matrix, and continues with the CNOT gate which can be represented by the matrix:

$C N O T=|0\rangle\langle 0|\otimes I+| 0\rangle\langle 0| \otimes \sigma_{x}=\left[\begin{array}{cccc}1 & 0 & 0 & 0 \\ 0 & 1 & 0 & 0 \\ 0 & 0 & 0 & 1 \\ 0 & 0 & 1 & 0\end{array}\right]$

where $\sigma_{x}$ is one of the three Pauli's matrices $\left(\sigma_{x}, \sigma_{y}, \sigma_{z}\right)[51]$

$\sigma_{x}=\left[\begin{array}{ll}0 & 1 \\ 1 & 0\end{array}\right], \quad \sigma_{y}=\left[\begin{array}{cc}0 & -i \\ i & 0\end{array}\right] \quad$ and $\quad \sigma_{z}=\left[\begin{array}{cc}1 & 0 \\ 0 & -1\end{array}\right]$

with $i=\sqrt{-1}$. Figure 6.3 shows both gates, Hadamard $(H)$ and CNOT [51] in that order. Then, using the Hadamard matrix of equation (6.6) and $|00\rangle$ from equation (2.10), we will have,

$(I \otimes H)|00\rangle=\frac{1}{\sqrt{2}}\left[\begin{array}{cccc}1 & 0 & 1 & 0 \\ 0 & 1 & 0 & 1 \\ 1 & 0 & -1 & 0 \\ 0 & 1 & 0 & -1\end{array}\right]\left[\begin{array}{l}1 \\ 0 \\ 0 \\ 0\end{array}\right]=\left[\begin{array}{c}1 / \sqrt{2} \\ 0 \\ 1 / \sqrt{2} \\ 0\end{array}\right]$

Finally, the CNOT gate of equation (6.7) is applied

$$
\begin{aligned}
{\left[\begin{array}{llll}
1 & 0 & 0 & 0 \\
0 & 1 & 0 & 0 \\
0 & 0 & 0 & 1 \\
0 & 0 & 1 & 0
\end{array}\right]\left[\begin{array}{c}
1 / \sqrt{2} \\
0 \\
1 / \sqrt{2} \\
0
\end{array}\right] } & =\left[\begin{array}{c}
1 / \sqrt{2} \\
0 \\
0 \\
1 / \sqrt{2}
\end{array}\right]=\frac{1}{\sqrt{2}}\left[\begin{array}{l}
1 \\
0 \\
0 \\
0
\end{array}\right]+\frac{1}{\sqrt{2}}\left[\begin{array}{l}
0 \\
0 \\
0 \\
1
\end{array}\right]=\frac{1}{\sqrt{2}}|0\rangle \otimes|0\rangle+\frac{1}{\sqrt{2}}|1\rangle \otimes|1\rangle \\
& =\frac{1}{\sqrt{2}}|0\rangle|0\rangle+\frac{1}{\sqrt{2}}|1\rangle|1\rangle=\frac{1}{\sqrt{2}}(|00\rangle+|11\rangle)=\left|\beta_{00}\right\rangle
\end{aligned}
$$


The Bell's bases as $\left|\beta_{00}\right\rangle$ are maximally entangled quantum states [1-3]. On the other hand, a reverse protocol to the equations (6.9) and (6.10) is applied to obtain the simple bases of equation (2.10), which we can obtain from the basis of Bell's equation (2.11), and which is used in quantum teleportation and superdense coding [53-59]. Such reverse protocol can be seen in Figure 6.4 and begins with a multiplication between the CNOT gate as a matrix, and $\left|\beta_{00}\right\rangle$

$$
\left[\begin{array}{llll}
1 & 0 & 0 & 0 \\
0 & 1 & 0 & 0 \\
0 & 0 & 0 & 1 \\
0 & 0 & 1 & 0
\end{array}\right]\left[\begin{array}{c}
1 / \sqrt{2} \\
0 \\
0 \\
1 / \sqrt{2}
\end{array}\right]=\left[\begin{array}{c}
1 / \sqrt{2} \\
0 \\
1 / \sqrt{2} \\
0
\end{array}\right]
$$

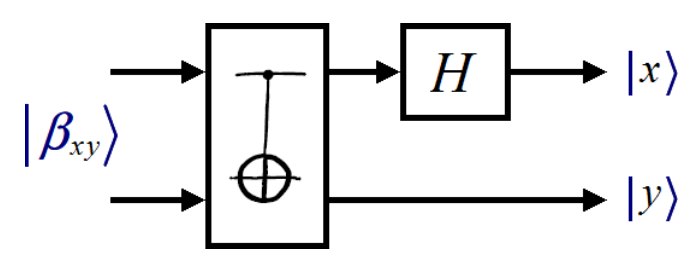

Figure 6.4 The input of these gates, CNOT and Hadamard $(H)$, is an EPR qubit or Bell's state, which at the output is decomposed into the respective Computational Basis States (CBS).

Now, applying the Hadamard's matrix to the result of the equation (6.11), we will have,

$$
\frac{1}{\sqrt{2}}\left[\begin{array}{cccc}
1 & 0 & 1 & 0 \\
0 & 1 & 0 & 1 \\
1 & 0 & -1 & 0 \\
0 & 1 & 0 & -1
\end{array}\right]\left[\begin{array}{c}
1 / \sqrt{2} \\
0 \\
1 / \sqrt{2} \\
0
\end{array}\right]=\left[\begin{array}{l}
1 \\
0 \\
0 \\
0
\end{array}\right]=|00\rangle=|0\rangle \otimes|0\rangle
$$

In this way, we obtained the original simple bases. With these deductions, we will begin to develop the protocols.

\section{Standard Quantum Teleportation}

\section{Noiseless analysis}

This protocol begins with the generation of an EPR pair, e.g., $\left|\beta_{00}\right\rangle \equiv\left|\Phi_{+}^{A \cup B}\right\rangle$ through the procedure of Figure 6.3, and with the subsequent distribution of the elements of said pair: one element to Alice and the other one to Bob, as we can see in Figure 6.5. Besides, the protocol is completed with a block not mentioned so far: $S P D$ (single photon detectors).

Now, If $\left|\psi_{0}\right\rangle=|\psi\rangle=\alpha|0\rangle+\beta|1\rangle$ is an arbitrary and unknown state to be teleported with $|\alpha|^{2}+|\beta|^{2}=1$ and $\alpha \wedge \beta \in \mathbb{C}$ of a Hilbert space, then, the initial state (3-partite state) will be,

$$
\begin{aligned}
\left|\psi_{0}\right\rangle & =|\psi\rangle \otimes\left|\beta_{00}\right\rangle=|\psi\rangle\left|\beta_{00}\right\rangle=(\alpha|0\rangle+\beta|1\rangle) 1 / \sqrt{2}(|00\rangle+|11\rangle) \\
& =1 / \sqrt{2}[\alpha|0\rangle(|00\rangle+|11\rangle)+\beta|1\rangle(|00\rangle+|11\rangle)] \\
& =1 / \sqrt{2}[\alpha|000\rangle+\alpha|011\rangle+\beta|100\rangle+\beta|111\rangle]
\end{aligned}
$$

where for simplicity, and from here on, in a generic form $|x\rangle \otimes|y\rangle=|x\rangle|y\rangle$. 


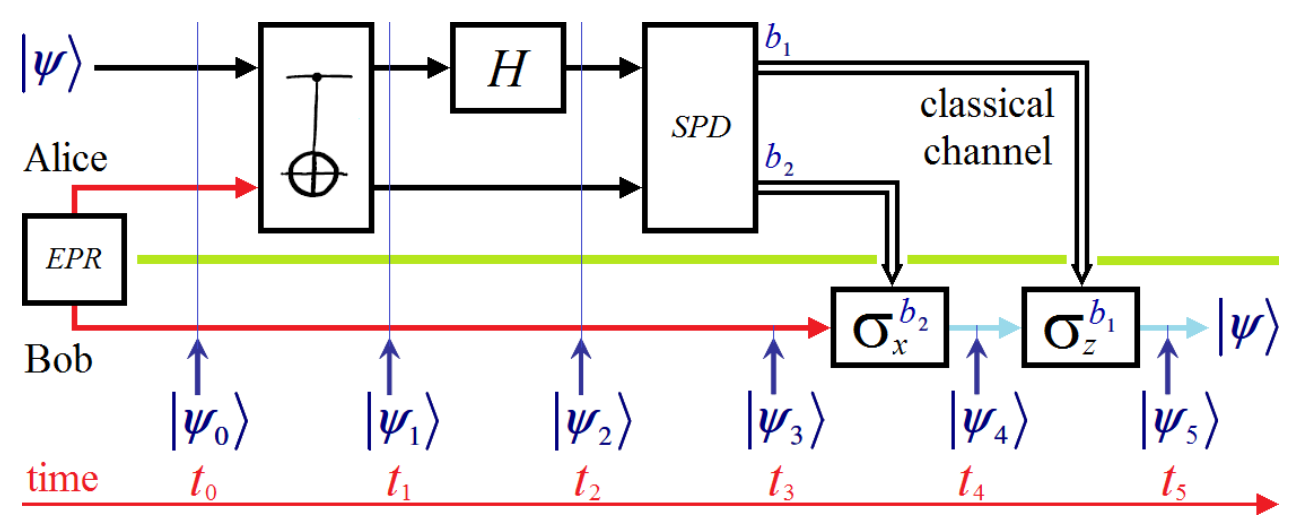

Figure 6.5 Standard Teleportation protocol using an EPR pair and two classical bits for disambiguation.

Now, the CNOT gate is applied to equation (6.13),

$\left|\psi_{1}\right\rangle=\frac{1}{\sqrt{2}}[\alpha|000\rangle+\alpha|011\rangle+\beta|110\rangle+\beta|101\rangle]$

In practice, Kronecker's product and CNOT gate are implemented together on the same beamsplitter $[55,57,58]$. At this time, we apply a Hadamard's gate to the elements of equation (6.14),

$$
\begin{aligned}
\left|\psi_{2}\right\rangle & =\frac{1}{2}\left[|00\rangle \sigma_{x}^{0} \sigma_{z}^{0}|\psi\rangle+|01\rangle \sigma_{x}^{1} \sigma_{z}^{0}|\psi\rangle+|10\rangle \sigma_{x}^{0} \sigma_{z}^{1}|\psi\rangle+|11\rangle \sigma_{x}^{1} \sigma_{z}^{1}|\psi\rangle\right] \\
& =\frac{1}{2}\left[\left|\Phi^{+}\right\rangle \sigma_{x}^{0} \sigma_{z}^{0}|\psi\rangle+\left|\Phi^{-}\right\rangle \sigma_{x}^{0} \sigma_{z}^{1}|\psi\rangle+\left|\Psi^{+}\right\rangle \sigma_{x}^{1} \sigma_{z}^{0}|\psi\rangle+\left|\Psi^{-}\right\rangle \sigma_{x}^{1} \sigma_{z}^{1}|\psi\rangle\right]
\end{aligned}
$$

In Table 6.1 we can see that Alice measures two of the possible qubits of the basis of equation (2.11); therefore, she transmits the corresponding bits $b_{1}$ and $b_{2}$ via a classical channel to Bob. The quantum measurement process is necessary to be carried out, in order not to violate the No-Cloning Theorem. In other words, the quantum measurement process destroys the entanglement [22, 51].

Table 6.1 Alice's side: measurement of base, classical transmission of bits, and collapse of states, Bob's side: classical reception of bits, gates application for the final recovery of the arbitrary state.

\begin{tabular}{ccccc}
\hline $\begin{array}{c}\text { Alice's } \\
\text { measurement }\end{array}$ & $\begin{array}{c}\text { Alice } \\
\text { transmits }\end{array}$ & $\begin{array}{c}\text { This happens with } \\
\text { a probability }\end{array}$ & Collapsed state & Bob applies $\sigma_{x}^{b_{1}} \sigma_{z}^{b_{2}}$ \\
\hline$\left|\Phi^{+}\right\rangle \rightarrow 00$ & $\mathrm{~b}_{2} \mathrm{~b}_{1}=00$ & $\| \frac{1}{2} \sigma_{x}^{0} \sigma_{z}^{0}|\psi\rangle \|^{2}=\frac{1}{4}$ & $\left|\Phi^{+}\right\rangle \sigma_{x}^{0} \sigma_{z}^{0}|\psi\rangle$ & $\sigma_{x}^{0} \sigma_{z}^{0}|\psi\rangle=|\psi\rangle$ \\
$\left|\Psi^{+}\right\rangle \rightarrow 01$ & $\mathrm{~b}_{2} \mathrm{~b}_{1}=01$ & $\| \frac{1}{2} \sigma_{x}^{1} \sigma_{z}^{0}|\psi\rangle \|^{2}=\frac{1}{4}$ & $\left|\Psi^{+}\right\rangle \sigma_{x}^{1} \sigma_{z}^{0}|\psi\rangle$ & $\sigma_{x}^{1} \sigma_{z}^{0}|\psi\rangle=\sigma_{x}|\psi\rangle$ \\
$\left|\Phi^{-}\right\rangle \rightarrow 10$ & $\mathrm{~b}_{2} \mathrm{~b}_{1}=10$ & $\| \frac{1}{2} \sigma_{x}^{0} \sigma_{z}^{1}|\psi\rangle \|^{2}=\frac{1}{4}$ & $\left|\Phi^{-}\right\rangle \sigma_{x}^{0} \sigma_{z}^{1}|\psi\rangle$ & $\sigma_{x}^{0} \sigma_{z}^{1}|\psi\rangle=\sigma_{z}|\psi\rangle$ \\
$\left|\Psi^{-}\right\rangle \rightarrow 11$ & $\mathrm{~b}_{2} \mathrm{~b}_{1}=11$ & $\| \frac{1}{2} \sigma_{x}^{1} \sigma_{z}^{1}|\psi\rangle \|^{2}=\frac{1}{4}$ & $\left|\Psi^{-}\right\rangle \sigma_{x}^{1} \sigma_{z}^{1}|\psi\rangle$ & $\sigma_{x}^{1} \sigma_{z}^{1}|\psi\rangle=\sigma_{x} \sigma_{z}|\psi\rangle$ \\
\hline
\end{tabular}

Bob applies two unit transformations $\sigma_{x}^{b_{1}} \sigma_{z}^{b_{2}}$ on his side according to the bits $\mathrm{b}_{2} \mathrm{~b}_{1}$ transmitted by Alice and, in this way, Bob recovers the teleported and arbitrary state $|\psi\rangle$.

\section{Noisy analysis}

Starting again from Figure 6.5, and considering noise in the EPR pair due to a disturbance of the shape $\left|\beta_{00}\right\rangle_{n}=A|00\rangle+B|11\rangle$

where subscript $n$ means noise, and 


$$
|A|^{2}+|B|^{2}=1 \text {, with }(A \neq B) \wedge(A \neq 1 / \sqrt{2}) \wedge(B \neq 1 / \sqrt{2})
$$

Then, repeating equation (6.13) but with $\left|\beta_{00}\right\rangle_{n}$ instead of $\left|\beta_{00}\right\rangle$, we will have

$$
\begin{aligned}
\left|\psi_{0}\right\rangle=|\psi\rangle\left|\beta_{00}\right\rangle_{n} & =(\alpha|0\rangle+\beta|1\rangle)(A|00\rangle+B|11\rangle) \\
& =\alpha A|000\rangle+\beta A|100\rangle+\alpha B|011\rangle+\beta B|111\rangle
\end{aligned}
$$

Now, the CNOT gate is applied to equation (6.18),

$\left|\psi_{1}\right\rangle=\alpha A|000\rangle+\beta A|110\rangle+\alpha B|011\rangle+\beta B|101\rangle$

At this time, we apply a Hadamard's gate to the elements of equation (6.19),

$$
\begin{aligned}
\left|\psi_{2}\right\rangle & =\frac{1}{\sqrt{2}}[\alpha A|000\rangle+\alpha A|100\rangle+\beta A|010\rangle-\beta A|110\rangle+\alpha B|011\rangle+\alpha B|111\rangle+\beta B|001\rangle-\beta B|101\rangle] \\
& =\frac{A}{\sqrt{2}}|00\rangle \alpha|0\rangle+\frac{B}{\sqrt{2}}|00\rangle \beta|1\rangle+\frac{A}{\sqrt{2}}|10\rangle \alpha|0\rangle-\frac{B}{\sqrt{2}}|10\rangle \beta|1\rangle+\frac{A}{\sqrt{2}}|01\rangle \beta|0\rangle+\frac{B}{\sqrt{2}}|01\rangle \alpha|1\rangle+\frac{B}{\sqrt{2}}|11\rangle \alpha|1\rangle-\frac{A}{\sqrt{2}}|11\rangle \beta|0\rangle \\
& =|00\rangle\left(\frac{A}{\sqrt{2}} \alpha|0\rangle+\frac{B}{\sqrt{2}} \beta|1\rangle\right)+|10\rangle\left(\frac{A}{\sqrt{2}} \alpha|0\rangle-\frac{B}{\sqrt{2}} \beta|1\rangle\right)+|01\rangle\left(\frac{B}{\sqrt{2}} \alpha|1\rangle+\frac{A}{\sqrt{2}} \beta|0\rangle\right)+|11\rangle\left(\frac{B}{\sqrt{2}} \alpha|1\rangle-\frac{A}{\sqrt{2}} \beta|0\rangle\right)
\end{aligned}
$$

From this point, we will follow a procedure similar to that of Table $\mathbf{6 . 1}$ but taking into account how the state is sensitively affected by noise.

\section{Simplified Quantum Teleportation}

This protocol does not require a classic channel to transmit disambiguation bits or the use of Pauli's matrices in the Bob's side in order to reconstruct the teleported state [165]. Figure 6.6 represents this protocol where block " $|0\rangle$ reset" does a strict reset of the qubit. Unlike the standard version of Figure 6.5, it uses $\left|\beta_{00}\right\rangle \otimes|\psi\rangle$ instead of $|\psi\rangle \otimes\left|\beta_{00}\right\rangle$ thanks to a $S W A P$ gate.

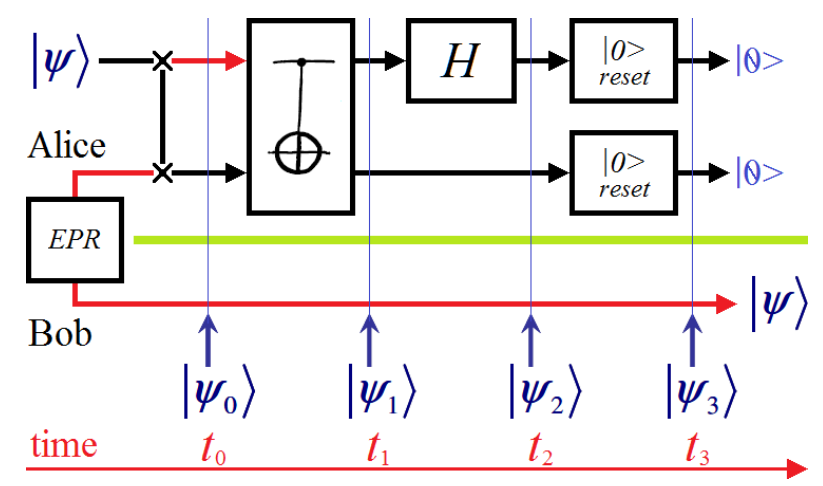

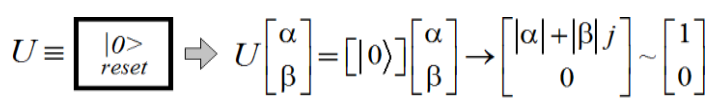

As a matrix, the $U$ gate:

- does not have reverse

- is not unitary

- converts any qubit in a ground state $\mid O>$

Figure 6.6 Simplified Teleportation protocol using an EPR pair but without classical bits for disambiguation.

\section{Noiseless analysis}

$$
\begin{aligned}
\left|\psi_{0}\right\rangle=\left|\beta_{00}\right\rangle|\psi\rangle & =\frac{1}{\sqrt{2}}(|00\rangle+|11\rangle)(\alpha|0\rangle+\beta|1\rangle) \\
& =\frac{1}{\sqrt{2}}[\alpha|000\rangle+\beta|001\rangle+\alpha|110\rangle+\beta|111\rangle]
\end{aligned}
$$


Now, the CNOT gate is applied to equation (6.21), and the result will be present on the Alice's lower branch, because in her upper branch will be $\left|\beta_{00}\right\rangle$,

$$
\begin{aligned}
\left|\psi_{1}\right\rangle & =\frac{1}{\sqrt{2}}[\alpha|000\rangle+\beta|001\rangle+\alpha|100\rangle+\beta|101\rangle]=\frac{1}{\sqrt{2}}[|00\rangle(\alpha|0\rangle+\beta|1\rangle)+|10\rangle(\alpha|0\rangle+\beta|1\rangle)] \\
& =\frac{1}{\sqrt{2}}(\alpha|0\rangle+\beta|1\rangle)[|00\rangle+|10\rangle]=(\alpha|0\rangle+\beta|1\rangle)\left[\frac{1}{\sqrt{2}}(|0\rangle+|1\rangle)\right]|0\rangle=|\psi\rangle|+\rangle|0\rangle
\end{aligned}
$$

It is evident from Figure 6.6 that the Hadamard's gate only involves to the Alice's upper branch, then,

$$
(I \otimes H)\left|\beta_{00}\right\rangle=\left(\left[\begin{array}{ll}
1 & 0 \\
0 & 1
\end{array}\right] \otimes \frac{1}{\sqrt{2}}\left[\begin{array}{cc}
1 & 1 \\
1 & -1
\end{array}\right]\right) \frac{1}{\sqrt{2}}(|00\rangle+|11\rangle)=\frac{1}{2}(|00\rangle+|10\rangle+|01\rangle-|11\rangle)
$$

Equation (6.22) shows that no disambiguation is necessary. Alice blocks her two branch thanks to a qubit reset gate $[\mid 0>]$ pair in order to annul all the projections with $\mid 1>$ components in equations (6.22) and (6.23). The No-Cloning Theorem $[1,166]$ is never violated. We can also see in Figure 5 that it is not necessary for Bob to apply any unitary transformation. This eliminates the classical channel that is responsible for making teleportation as a whole to be carried out in a time greater than zero, i.e., not being instantaneous. This protocol represents the verification of everything said in this work.

\section{Noisy analysis}

For noisy EPR pairs we also resorted to Figure 6.6 using the same version of equations (6.16) and (6.17). Then, repeating equation (6.21) but with $\left|\beta_{00}\right\rangle_{n}$ instead of $\left|\beta_{00}\right\rangle$, we will have

$$
\begin{aligned}
\left|\psi_{0}\right\rangle=\left|\beta_{00}\right\rangle_{n}|\psi\rangle & =(A|00\rangle+B|11\rangle)(\alpha|0\rangle+\beta|1\rangle) \\
& =A \alpha|000\rangle+B \alpha|110\rangle+A \beta|001\rangle+B \beta|111\rangle
\end{aligned}
$$

Now, we apply the CNOT gate to equation (6.24), and the result will be present on the Alice's lower branch again, because in her upper branch will be $\left|\beta_{00}\right\rangle$,

$$
\begin{aligned}
\left|\psi_{1}\right\rangle & =A \alpha|000\rangle+B \alpha|100\rangle+A \beta|001\rangle+B \beta|101\rangle=A|00\rangle(\alpha|0\rangle+\beta|1\rangle)+B|10\rangle(\alpha|0\rangle+\beta|1\rangle) \\
& =(A|00\rangle+B|10\rangle)(\alpha|0\rangle+\beta|1\rangle)=(A|00\rangle+B|10\rangle)|\psi\rangle=C|\psi\rangle
\end{aligned}
$$

where

$$
C=(A|00\rangle+B|10\rangle)
$$

Newly, the Hadamard's gate only involves to the Alice's upper branch, exclusivelly, which can be seen in Figure 6.6, then,

$$
\begin{aligned}
(I \otimes H)\left|\beta_{00}\right\rangle_{n} & =\left(\left[\begin{array}{ll}
1 & 0 \\
0 & 1
\end{array}\right] \otimes \frac{1}{\sqrt{2}}\left[\begin{array}{cc}
1 & 1 \\
1 & -1
\end{array}\right]\right)(A|00\rangle+B|11\rangle) \\
& =\frac{1}{\sqrt{2}}(A|00\rangle+A|10\rangle+B|01\rangle-B|11\rangle)
\end{aligned}
$$

The worst consequence of noise in the new protocol is that the teleported state loses its purity, which means, it would not be on the Bloch's sphere, given that in the more general case, $C \neq 1$, however, the teleported state is recovered without any problems or disambiguation. This clearly indicates that the new protocol is much more robust (immune to noise) than the standard. 


\section{Standard Superdense Coding (SSDC)}

Before starting, we will establish a clear differentiation on the type of link that each protocol will use:

- Classical channel: it is any traditional link of the electromagnetic type (radial, satellite or cable), smoke signals, letters or carrier pigeons,

- Quantum channel: we usually refer to this link when it comes to fiber optic, or any other type of optical link, and finally,

- Entanglement link: it is the one that is established thanks to an EPR pair previously distributed between, e.g., Alice and Bob, which can be carried out through two possible procedures: delivery or take-out.

What does delivery or take-out mean? This can be explained with an example. Let us suppose that in a trip to Mars, the crew of the ship reaches the orbit of the red planet and needs to send news and queries to Earth. The average distance between the two planets is 225 million kilometers. Light travels at approximately $299,792 \mathrm{~km} / \mathrm{s}$. Therefore, a message sent from the surface of Mars (via a traditional radial transmission) would take a little more than 3 minutes to reach Earth when they are at their closest, or 22 minutes if the planets are further away as long as the Earth's antenna can be visible from Mars, the ship is on the visible side of Mars and no other celestial sphere intervenes. However, as the communication will take place through a link based on entanglement, we must distribute an EPR pair between Earth and the ship at some point, that is, we have two options:

- take-out: before the ship leaves the Earth, we introduce one of the elements of the EPR pair into a specially designed holder, while the other one remains on Earth, or

- delivery: once the ship reaches the orbit of Mars, and as long as nothing is optically interposed in the middle, we send an element of the EPR pair from Earth to Mars through any optical technology.

Once each party has its own EPR, we are not concerned with alignments, hidden faces of the planets or other stars in the middle. However, there are some practical problems involved in all the examples mentioned:

- the lifetime of an EPR pair, depending on the technology chosen to create the qubits, could reach at best some 10 milliseconds [167], and the trip to Mars, at best, would take six months, i.e., the takeout option, right now, does not work,

- the best optical technology would take at least 3 minutes to make a delivery of the EPR element from Earth to Mars. Therefore, the delivery option does not work for now either for the same reason as the previous case. Unfortunately, today the main enemies of the entanglement are the decoherence and the short lifetime of the qubits that compose it.

- during a teleportation act, the entanglement is eliminated once a Bell State Measurement (BSM) is performed as part of the procedure to recover the teleported state to avoid violating the No-Cloning Theorem. Consequently, we need to get another EPR pair on each side (Alice and Bob) in order to teleport the next arbitrary state. This requires a new distribution of an EPR pair which is always subject to the speed of light. If we now decide to apply an entanglement recovery procedure like the one suggested in $§ 5.5$, the question is: what are we recovering? Are we recovering the EPR pair or a mix between this pair and the last teleported arbitrary state? And in this last case, would we be violating the No-Cloning Theorem? Anyways, the instantaneity of the entanglement (i.e., its principal attribute) does not serve us yet for several reasons: the BSM, the lifetime of the qubits, and any other possible technological problem, since we are still subject to the speed of light. That is, we have a series of technical problems in addition to the entanglement itself that complicate any practical implementation of a link based on it. However, if such problems did not exist, once each side of the link had its element of the EPR pair, nothing would get in the way. 
There is only one thing that can save us: the entanglement between particles that never coexisted spatially. If this technique existed, we could generate non-ubiquitous EPR pairs in a completely disruptive and instantaneous way without violating the No-Cloning Theorem. But in order to achieve this, we need to know, understand and control entanglement more in depth.

Finally, and returning to superdense coding, we can see this protocol in Figure 6.7.

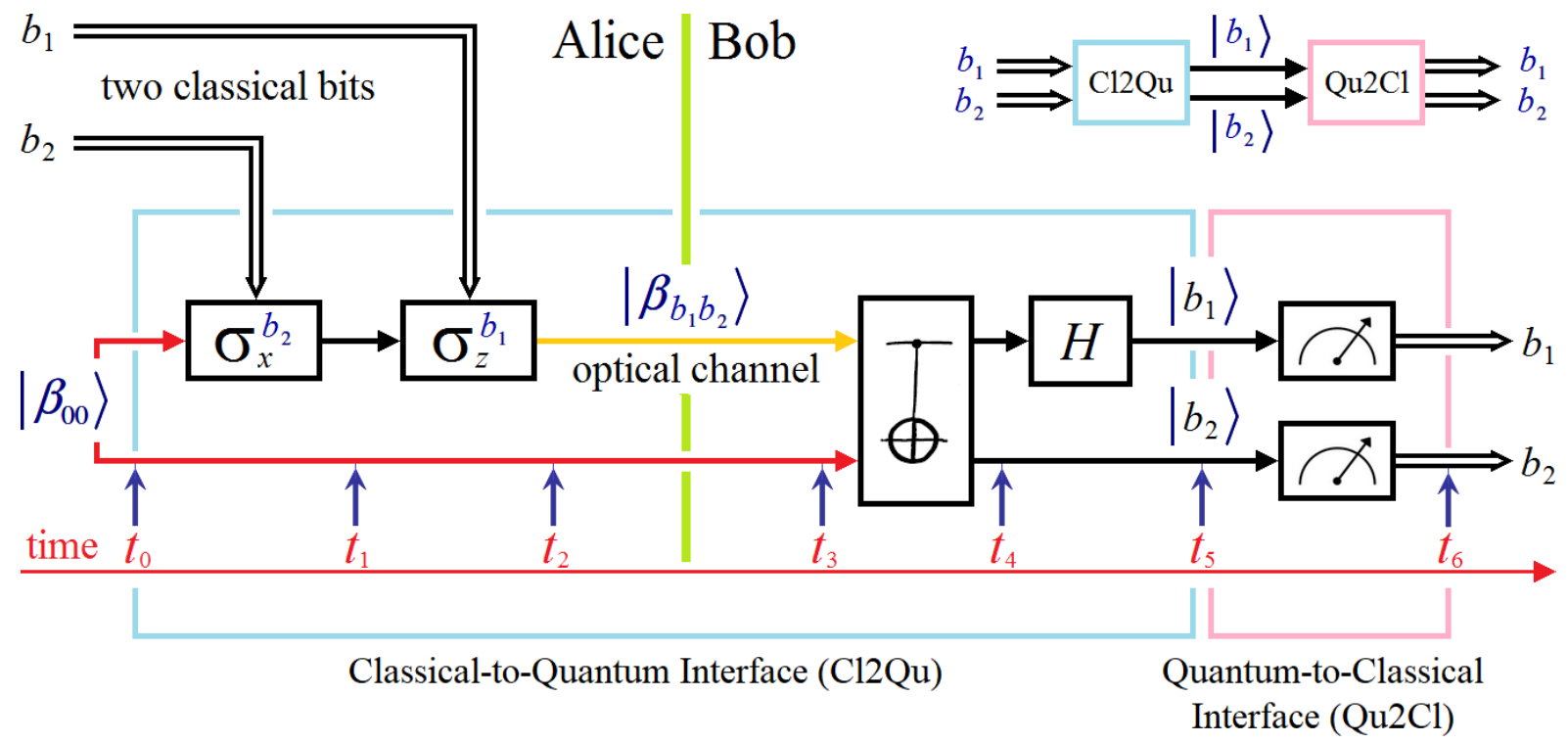

Figure 6.7 Standard Superdense Coding and the detail of its constituent interfaces.

We begin with both possibilities according to $b_{2}$, i.e., 0 or 1 . If $b_{2}=0$, then, in $t_{1}$ we have $\left|\beta_{00}\right\rangle$ again. But, if $b_{2}=1$, then, in $t_{1}$ we have,

$\left(I \otimes \sigma_{x}\right)\left|\beta_{00}\right\rangle=\left(\left[\begin{array}{ll}1 & 0 \\ 0 & 1\end{array}\right] \otimes\left[\begin{array}{ll}0 & 1 \\ 1 & 0\end{array}\right]\right)\left[\begin{array}{c}1 / \sqrt{2} \\ 0 \\ 0 \\ 1 / \sqrt{2}\end{array}\right]=\left[\begin{array}{llll}0 & 0 & 1 & 0 \\ 0 & 0 & 0 & 1 \\ 1 & 0 & 0 & 0 \\ 0 & 1 & 0 & 0\end{array}\right]\left[\begin{array}{c}1 / \sqrt{2} \\ 0 \\ 0 \\ 1 / \sqrt{2}\end{array}\right]=\left[\begin{array}{c}0 \\ 1 / \sqrt{2} \\ 1 / \sqrt{2} \\ 0\end{array}\right]$

if in $t_{2}, b_{2}=0$ and $b_{1}=0$, we obtain $\left|\beta_{00}\right\rangle$. But if $b_{2}=0$ and $b_{1}=1$, we will have,

$\left(I \otimes \sigma_{z}\right)\left|\beta_{00}\right\rangle=\left(\left[\begin{array}{ll}1 & 0 \\ 0 & 1\end{array}\right] \otimes\left[\begin{array}{cc}1 & 0 \\ 0 & -1\end{array}\right]\right)\left[\begin{array}{c}1 / \sqrt{2} \\ 0 \\ 0 \\ 1 / \sqrt{2}\end{array}\right]=\left[\begin{array}{cccc}1 & 0 & 0 & 0 \\ 0 & 1 & 0 & 0 \\ 0 & 0 & -1 & 0 \\ 0 & 0 & 0 & -1\end{array}\right]\left[\begin{array}{c}1 / \sqrt{2} \\ 0 \\ 0 \\ 1 / \sqrt{2}\end{array}\right]=\left[\begin{array}{c}1 / \sqrt{2} \\ 0 \\ 0 \\ -1 / \sqrt{2}\end{array}\right]$

Now, if $b_{2}=1$ and $b_{l}=1$, we will obtain,

$\left(I \otimes \sigma_{z}\right)\left[\begin{array}{c}0 \\ 1 / \sqrt{2} \\ 1 / \sqrt{2} \\ 0\end{array}\right]=\left(\left[\begin{array}{ll}1 & 0 \\ 0 & 1\end{array}\right] \otimes\left[\begin{array}{cc}1 & 0 \\ 0 & -1\end{array}\right]\right)\left[\begin{array}{c}0 \\ 1 / \sqrt{2} \\ 1 / \sqrt{2} \\ 0\end{array}\right]=\left[\begin{array}{cccc}1 & 0 & 0 & 0 \\ 0 & 1 & 0 & 0 \\ 0 & 0 & -1 & 0 \\ 0 & 0 & 0 & -1\end{array}\right]\left[\begin{array}{c}0 \\ 1 / \sqrt{2} \\ 1 / \sqrt{2} \\ 0\end{array}\right]=\left[\begin{array}{c}0 \\ 1 / \sqrt{2} \\ -1 / \sqrt{2} \\ 0\end{array}\right]$ 
The four obtained results, according to the values of $b_{2}$ and $b_{1}$, travel through the optical channel of Figure 6.7 under the generic name of $\left|\beta_{b_{1} b_{2}}\right\rangle$. From this point and applying the CNOT and Hadamard's $(H)$ gates as shown in Figure 6.4, we will obtain $\left|b_{1}\right\rangle$ and $\left|b_{2}\right\rangle$. Paying attention to the light blue rectangle of Figure 6.7, we can see that from $\left\{b_{2}, b_{1}\right\}$ to $\left\{\left|b_{1}\right\rangle,\left|b_{2}\right\rangle\right\}$ we have a classical-toquantum $(\mathrm{Cl} 2 \mathrm{Qu})$ interface for Computational Basis States (CBS) $\{0,1\} \rightarrow\{|0\rangle,|1\rangle\}$, respectively. Such interface can be seen in detail in Figure 6.8.

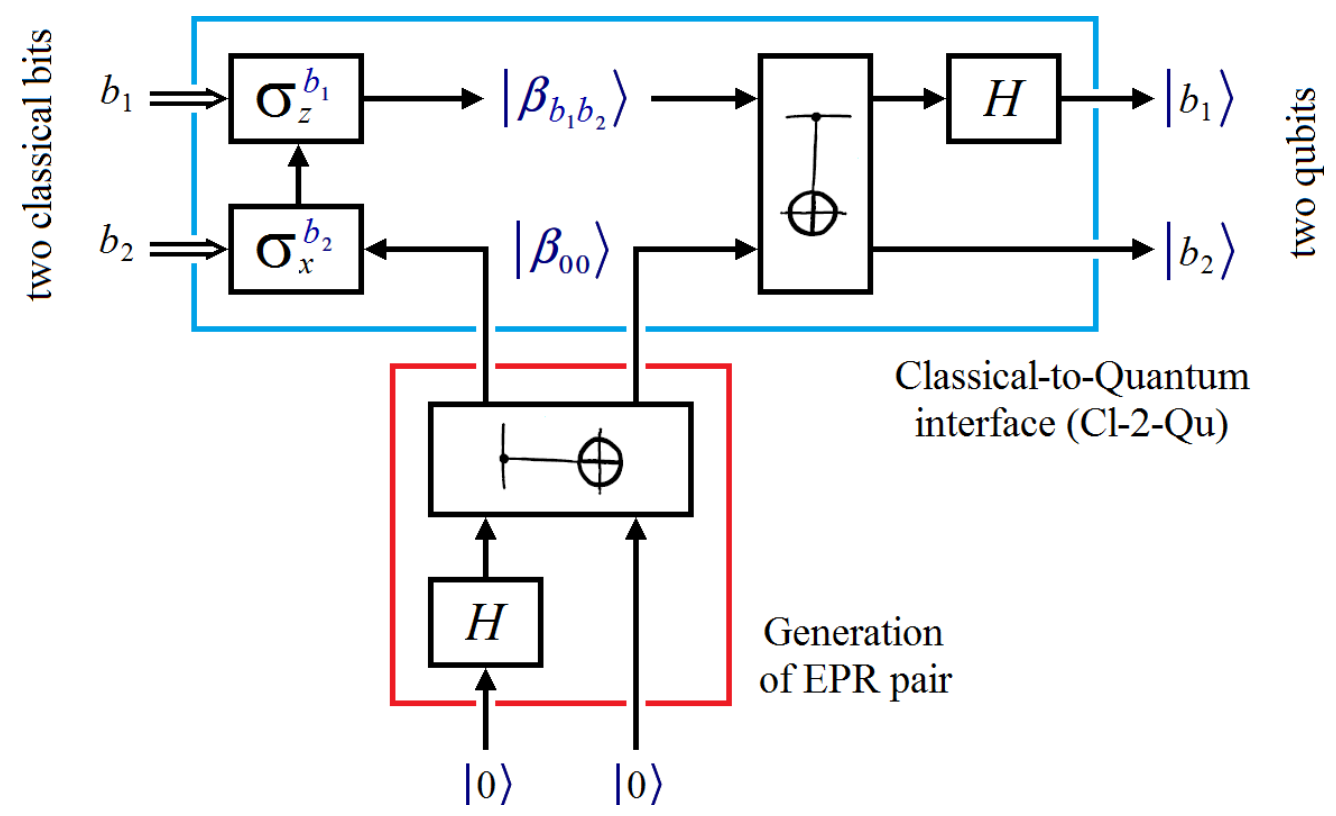

Figure 6.8 Classical-to-Quantum interface.

At the entrance of the pink rectangle in Figure 6.7, we have $\left\{\left|b_{1}\right\rangle,\left|b_{2}\right\rangle\right\}$, while at its exit we will have $\left\{b_{2}, b_{1}\right\}$, i.e., quantum measurement is a quantum-to-classical interface (Qu2Cl). With this sequence $\mathrm{Cl} 2 \mathrm{Qu}+\mathrm{Qu} 2 \mathrm{Cl}$ we complete the standard superdense coding protocol.

\section{Enhanced Superdense Coding}

In standard superdense coding, we need a quantum channel (optical fiber) to transmit $\left|\beta_{b_{1} b_{2}}\right\rangle$, i.e., this protocol is as subject to the speed of light as standard quantum teleportation is to the use of the classical channel to transmit the disambiguation bits. Therefore, to obtain an instantaneous superdense coding we will introduce the following:

1) both bits $\left\{b_{2}, b_{1}\right\}$ in a Cl2Qu interface like that of Figure 6.8. So we get $\left\{\left|b_{1}\right\rangle,\left|b_{2}\right\rangle\right\}$,

2) each qubit of $\left\{\left|b_{1}\right\rangle,\left|b_{2}\right\rangle\right\}$ in a simplified quantum teleportation like Figure 6.6,

3) both teleported qubits $\left\{\left|b_{1}\right\rangle,\left|b_{2}\right\rangle\right\}$ in a Qu2Cl interface (quantum measurement).

This protocol is complete in Figure 6.9. Such protocol allows us to transmit bits in a way that is not limited by the speed of light since it works with CBS which is highly efficient when recovering states from a quantum tomography [51] every time we make a measurement. Finally, once the technical limitations are overcome, this protocol will be the ideal method for a practical communication between the Earth and Mars. 


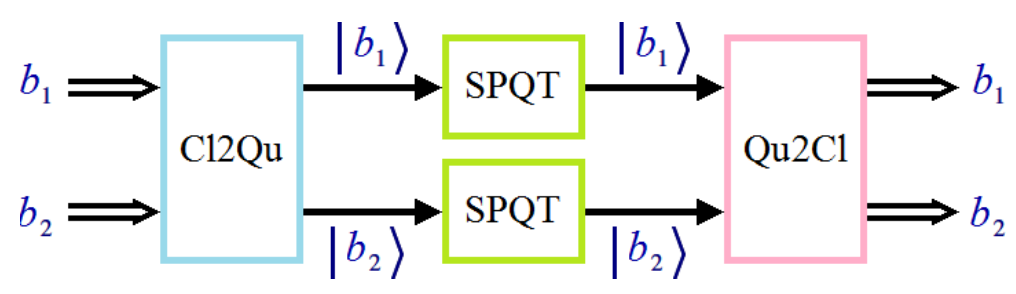

Figure 6.9 Enhanced superdense coding.

\subsection{Theory of Dilated Locality}

In Figure 6.10, we can see Alice's original spins in point $\mathrm{Y}$ and Bob's original spins in point $\mathrm{X}$, while the avatars are all together in point $\mathrm{Z}$.

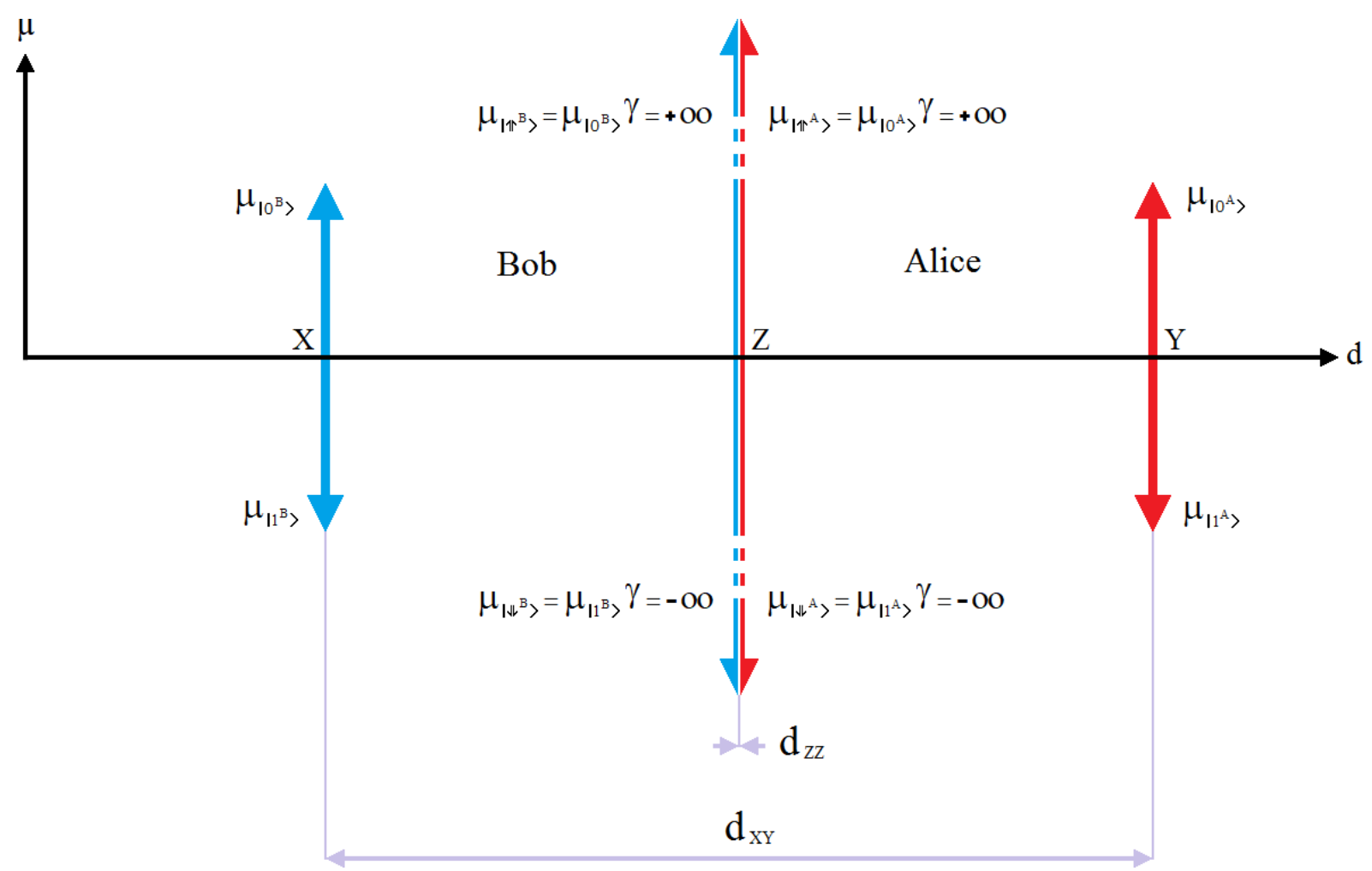

Figure 6.10 Avatars, original spins and the distances between them.

Let us suppose that Alice now performs a quantum measurement on her particle. At the same time all of the following facts happen:

- Alice knows the spin of her particle,

- Bob has a reverse spin (i.e., opposite orientation) to Alice's,

- quantum measurement destroys the entanglement,

- the avatars go from being 4 to being 2 and their values go from $\pm \infty$ to \pm 1 . This last case coincides with that one of two completely independent particles.

At the same time, a quasi-message of cancellation of entanglement passes from Alice to Bob through the plenipotentiary representatives of their original spins: the avatars, which are the ones that actually communicate since, as we know, this communication is instantaneous. As the original spins are not local to each other, they could never communicate instantaneously between them. 
Let us also suppose that this message is initially transmitted at the speed of light, therefore, the time it takes to transmit such cancellation message of the entanglement between avatars will be,

$$
\Delta t_{\text {avatars }}=\frac{d_{\mathrm{ZZ}}=0}{v=c}=0
$$

But being the distance between avatars null $d_{z Z}=0$, the speed $v$ could be much slower than the speed of light; however, the message would continue being instantaneous,

$$
\Delta t_{\text {avatars }}=\frac{d_{Z Z}=0}{v<<c}=0
$$

When we talk about avatars, there is no problem with the speed since they are local to each other, but Alice and Bob are not local to each other even though they are communicating instantaneously. Although the avatars are the ones who actually communicate, there will be a situation in which Alice communicates with her avatars, the avatars with each other, and Bob's avatars with him. If Alice and Bob are at a distance of Earth-Mars type, then the distance between Alice and her avatars is EarthMars divided by 2, so, how can the communication between Alice and Bob be instantaneous although it is the avatars who really communicate? In other words, the final effect is that Alice notifies Bob of the cancellation of the entanglement instantly even though both are mutually nonlocal. How can this be explained? Let us try to do it by adapting the equation (6.31) so that it can reflect this reality from the point of view of the non-locality of Alice and Bob, although it maintains the hypothesis of instantaneity, which we know it happens,

$$
\Delta t_{\text {avatars }}=\frac{d_{Z Z}=0}{v=c}=\frac{\left(d_{X Y} \gg 0 / \gamma\right)=0}{v=c}=\frac{d_{X Y} \gg 0}{v=c \gamma}=0=\Delta t_{\text {originals }}
$$

This equation simultaneously involves the avatars and the original spins' points of view. In the third member of the equation (6.33), we replace the inter-avatars distance by the distance between Alice's and Bob's spins conditioned by the homothecy of the equation (6.4). In the fourth member of equation (6.33), we go down the Lorentz factor to the denominator so that it gets involved with the speed leaving in the numerator the distance between Alice and Bob alone. Consequently, for an instantneous communication between Alice and Bob, like that one between avatars, the only possibility is that the homothecy also reaches the speed of light. As we know nothing exceeds the speed of light, so, what equation (6.33) is really telling us is that locality dilates like a balloon that is inflated, that is, we speak of an equivalent effect and not of an original reality.

Everything said so far can be seen in detail in Figure 6.11. In the upper part of this figure, we can see two entangled particles in the Alice's and Bob's hands at points A and B, respectively. We also see an intermediate point $Z$ where we know the avatars are. The lower part of Figure 6.11 shows us the combined effect analyzed above which is interpreted as if we had two equivalent giant particles of a diameter equal to the distance between Alice and Bob (potentially infinite), superimposed and centered at point $\mathrm{Z}$ and whose original spins are the avatars. They seem to be one giant particle because they are superimposed, but they are, actually, two. All this speaks to us of equivalent behaviors, exclusively, given that:

- nothing exceeds the speed of light,

- there are no two equivalent giant particles, and by the way,

- avatars do not really exist either.

Entanglement works as if the original spins behave like the avatars, and by behaving like them we can justify the extraordinary attributes of entanglement without crashing GR and QM. At the same time, the true equivalence between these theories is explained. 

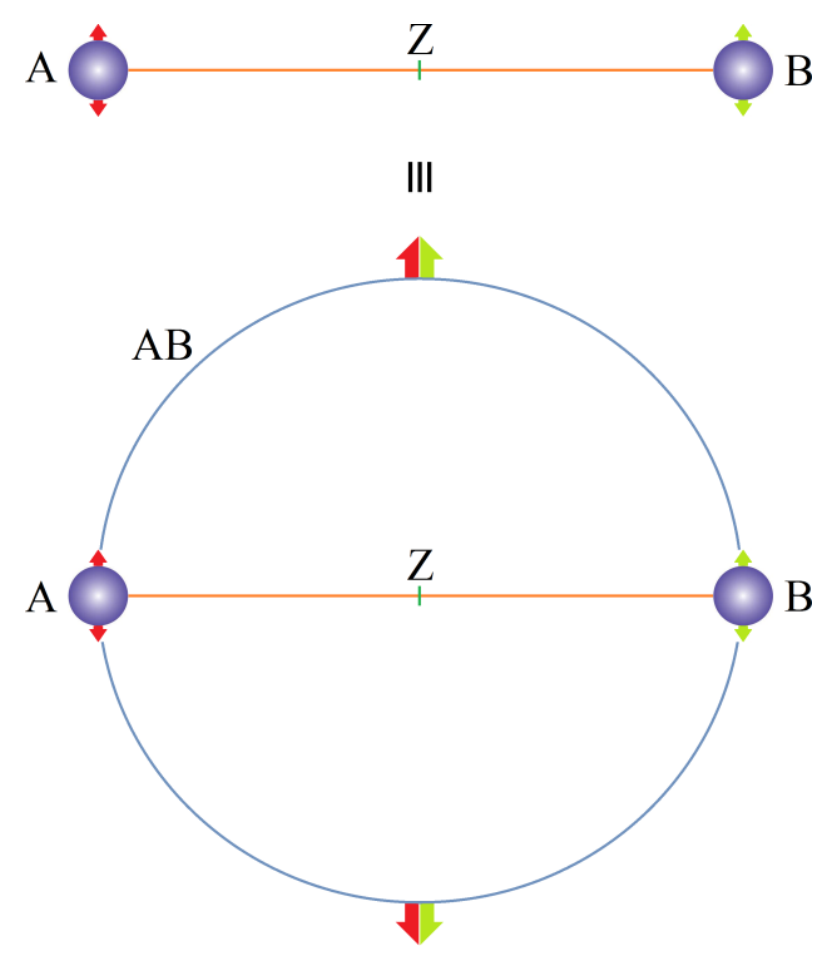

Figure 6.11 Theory of Dilated Locality.

Figure 6.11 resembles an interferometry effect, by which small things operatively arranged in an appropriate layout equate to a single larger thing. This happens in radio astronomy where a kilometer antenna is simulated thanks to small antennas, forming different patterns on land $[168,169]$ or in Synthetic Aperture Radar (SAR) where the movement of a small airborne or satellite antenna synthesizes an equivalent antenna of kilometers which never could be transported practically by plane or satellite [170]. Both the radio astronomy equivalent antenna and the SAR equivalent antenna do not really exist, however, we enjoy their benefits every day.

Besides, in Figure 6.11, the equivalent contraction of the distance between Alice and Bob is equivalent to the apparent dilatation of $c$. We can symbolize the dilatation of the locality of Alice and Bob as,

$$
\text { locality }_{\text {equivalent }}=\text { locality }_{\text {original }} \gamma
$$

Also, it is as if the past light cone of the original spins was dilated. Finally, all this leads us to an inevitable conclusion,

Non-locality $\equiv$ Dilated locality

All these meanings of Figure 6.11 explain why when we touch something from an entangled pair, we touch everything, which is absolutely consistent with the collective behavior of entanglement through the Bell's bases of equation (2.11) where individualities are completely displaced.

\subsection{Bell's Theorem and the avatars}

This subsection revolves around three questions:

- are the avatars some kind of hidden variables?

- if so, are they local or non-local?

- are they contextual or non-contextual?

We will try to answer them in order and in relation to Bell's Theorem [30]. 
By the way, we have talked a lot about Bell's Theorem but we have not mentioned any elements that will help us with these initial questions yet, in particular: hidden local variables, which are present in his theorem. To start with, the theorem springs from the following assumptions:

$$
A(a, \lambda)= \pm 1, \text { and } B(b, \lambda)= \pm 1
$$

where $A$ is the result of measuring $\sigma_{1} \cdot a . B$ is the result of measuring $\sigma_{2} \cdot b . \sigma_{1}$ and $\sigma_{2}$ are spins, while $a$ and $b$ are some unit vectors. Finally, $\lambda$ are the local hidden variables. Now, we will continue with the following definition:

$$
P(a, b)=\int \rho(\lambda) A(a, \lambda) B(b, \lambda) d \lambda
$$

Here, we have considered that if $\rho(\lambda)$ is the probability distribution of $\lambda$ then the expectation value of the product of the two components $\sigma_{1} \cdot a$ and $\sigma_{2} \cdot b$ is equation (6.37) which should equal the quantum mechanical expectation value. For the singlet state, it is

$$
\left\langle\sigma_{1} \cdot a, \sigma_{2} \cdot b\right\rangle=-a \cdot b
$$

but experimental implementations of the theorem [31, 32, 47, 49] have shown that this is not possible. Finally, it is necessary that

$$
\int \rho(\lambda) d \lambda=1
$$

$$
\rho(\lambda) \geq 0
$$

From equation (6.36) to (6.38) we can see that the avatars adapt to the assumption of equation (6.36) and to the definition of equation (6.37) without any problems, but, what about equation (6.39), which sets strict conditions for the hidden variables involved in Bell's Theorem? To answer this question, we must remember that there are four avatars, two positive and two negative ones. Assuming a discrete version of the equation (6.39a), the sum of the avatars in their space would be zero not one. Besides, with respect to the distribution of equation $(6.40 \mathrm{~b})$ half of the avatars would not fulfill this condition. Therefore, the answer to the first of the three initial questions of this subsection is that the avatars may be hidden variables, but not in Bell's sense. Finally, the avatars never establish the value of the physical quantity before the measurement, i.e., they are not hidden variables in the sense of the EPR paradox [21]. The value is determined only once it has been measured. In fact, the avatars change with the measurement, see Figure 5.17 of $§ 5.5$. They are simply a reflection of the original spins with extraordinary powers to represent them. They are a functional and operative equivalent thereof.

In regard to the second question, we can say that it is relatively easy to answer. Although the avatars are local to each other, they are hidden non-local variables in relation to the original spins, so they do not contradict the experimental results of Bell's Theorem [30]. But, if the locality is dilated, then, from an operational point of view, the avatars are all that exists and are local to each other. That is, they are local without contradicting the GR and without the need for a superluminal signaling. Finally, when we said that the avatars did not have problems with the equations (6.36) and (6.37), we were not talking about the fact that they behaved as hidden contextual variables; on the contrary, if they had not fulfilled the requirements of being a hidden variable, it would have been because of a reason much more powerful than that. If the avatars were hidden non-local variables (which makes them apparently incompatible with GR), then they would be contextual, but when the locality expands they become local (which makes them apparently incompatible with QM), so they should have previously been non-contextual. The reality is that thanks to the expansion of the locality, the avatars are a hinge in every sense, because they are local without contradicting the GR and are not local without contradicting the QM being compatible with the result of all experiments. 


\section{5. $G R=Q M$, quantum gravity and anti-gravity}

In [71], an invitation to demonstrate a correspondence between GR and QM through Quantum Information Processing's own procedures [51-53] in the upcoming decades is proposed. But, how could this be possible? Where does this prediction lead us to? What does he imply in his proposal? What clues support such a claim? Recall that although the avatars are a hinge between GR and QM, we never mention the word gravity in relation to them. Therefore, let us try to explore the substrate of this proposal by attempting to involve gravity in all this.

Figure 6.12 shows the four forces of the universe: gravity, electromagnetism, weak nuclear force, and strong nuclear force [20]. The link between the last three forces is already known, the problem is gravity. If we had to link them all, the blue node immediately after the Big Bang of Figure 6.12 would be the natural link for this purpose. Two branches emerge from this node: the one that leads to the gravitational force [171] and the one that makes it towards the Higgs' field [172-178] through which all the particles acquire their mass.

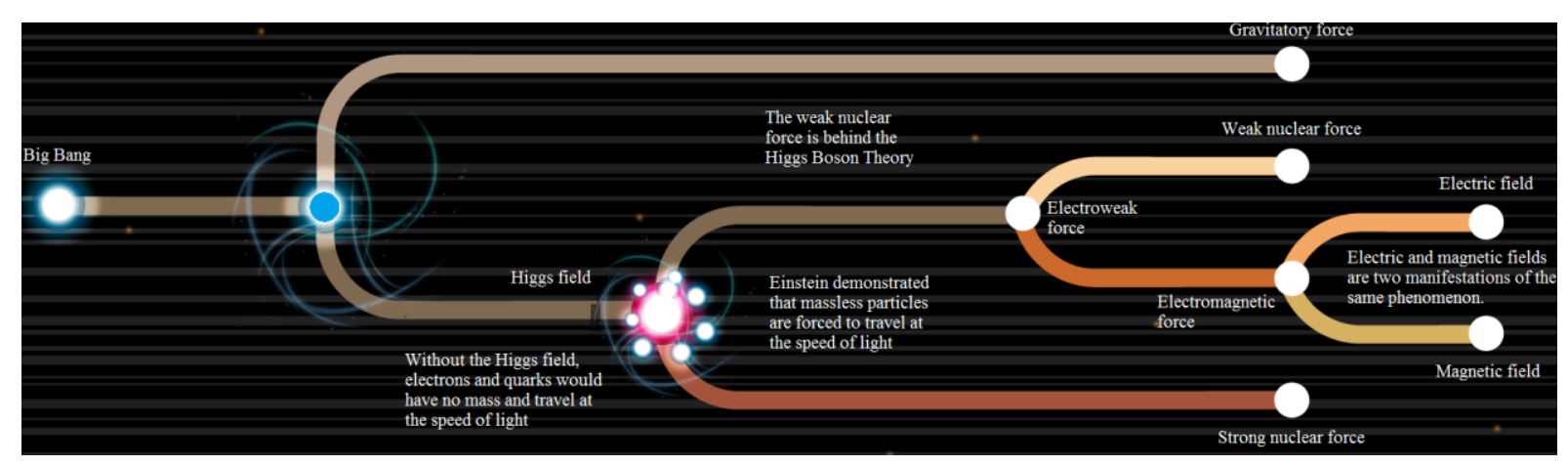

Figure 6.12 Forces of the universe.

In other words, before speaking of mass, there is something previous that deforms space-time [179182] giving rise to both branches. Maybe it is the spin and not the mass, as Einstein believed, that deforms space-time. Thus, we would conclude that the GR equation should be dependent on the spin instead of on the mass, as Figure 6.13 suggests. We will advance carefully with this slogan until we arrive at the analysis of geometric curvature around a supermassive object in terms of spins instead of the mass as in the original case of Schwarzschild [179].

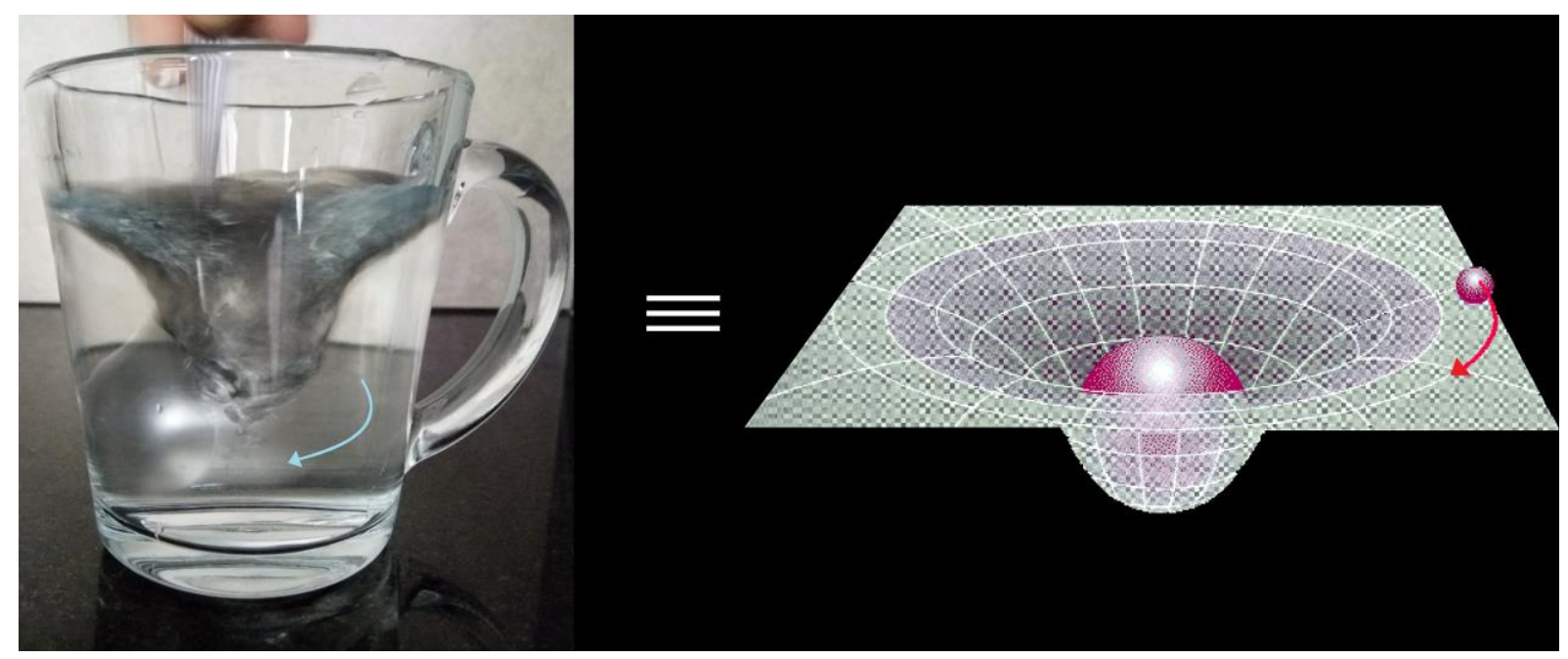

Figure 6.13 Spin as responsible for the curvature of space-time instead of mass. 
Together with QM, GR has been an absolutely successful theory for the last 100 years [183]. With different nuances and approaches, in which GR has had to interact even with different forms of spins [184], it has proved its validity. In fact, the GR-field-equation has been adapted to try to justify all the observable physical facts at a planetary and galactic level, with and without the famous cosmological constant [185-191] replacing the Newtonian notion of force with the deformation of space-time. In this last aspect, there have been many attempts to use GR-field-equation to justify the expansion of the universe [187-189]. Undoubtedly, the pioneer in this field has been Friedmann [192-196], who considered models with matter that expanded from a singularity of infinite density without the need to make force, the main actor of daily life, explicit. We know that GR apparently fails at the particle level [197], probably because of the weakness of gravity at that level. It is obvious that in this context there have not been many works in charge of linking GR with the particles' spin. Obviously, and by virtue of these circumstances, any approximation between gravity and entanglement becomes ephemeral.

If we return for a moment to the blue point of Figure 6.12, and go chronologically from left to right, so starting with the Big Bang, we can see that particles still do not have mass in this blue point since the Higgs' field with which said particles must interact to obtain their mass is not present yet. From Einstein we know that if the particles did not have mass (as in the case of the photon), then they would travel at the speed of light. Of course, but if in the blue point of Figure 6.12 the mass does not yet exist, how can we apply it to the Einstein's GR-field-equation? This reinforces the need for a version of the GR-field-equation independent of mass. The reality is that we have no idea what happens in the blue point in Figure 6.12. However, before issuing a trial, let us explore some options.

The question is: what tools do we have in the blue point of Figure 6.12 to be able to talk about attraction and eventually a force? The answer is: translations and spins. Therefore, let us try to define a force according to the latter trying to involve entanglement in that effort.

Let us try to model an attractive force between particles with the few tools that we have in the blue point of Figure 6.12, based on Figure 6.14. The model that we propose is replicated in all Physics where an attractive force (or not) intervenes between elements of similar characteristics, however, we perceive that something more is needed. In our opinion, that additional thing that is needed is entanglement. For example, if we characterize this force in terms of the spins, we must simultaneously consider that this force has a field automatically associated as in the case of Newton's gravitational field [171]. In the upper part of Figure 6.14, we can see two completely independent spins. In the middle of such spins an eventual field is counteracted because of their orientation. On the other hand, in the lower part of said figure the opposite happens, i.e., that supposed field is reinforced.

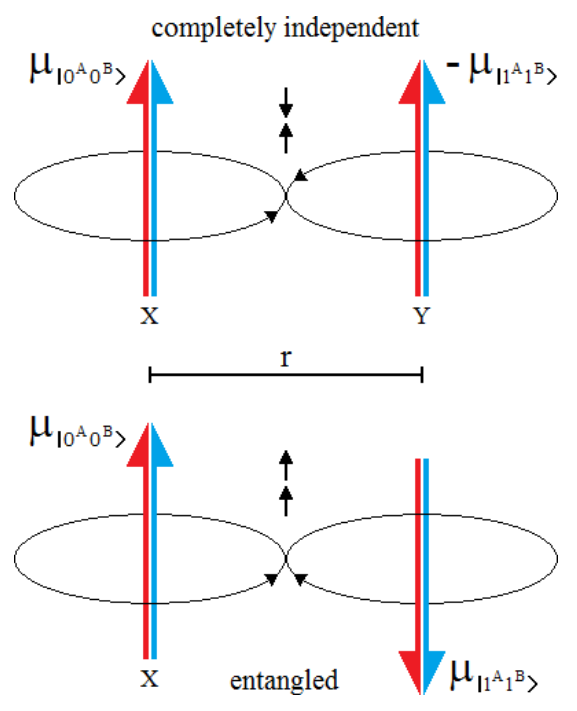

Figure 6.14 Upper part of figure: completely independent spins do not attract each other. Lower part of figure: entangled spins attract each other 
We can see that the spins in the lower part of Figure 6.14 are entangled. Let us try to put this in a set of equations, starting with force,

$$
F=\xi_{o} \frac{\left.-\mu_{\left|0^{A} 0^{B}\right\rangle} \mu_{\left|1^{A} 1^{B}\right\rangle}\right\rangle}{r^{2}} \vec{r}
$$

where $\vec{r}$ is a unitary vector that points in the direction that joins the spins, while $\xi_{o}$ is a constant with characteristics equivalent to Newton's universal gravitation constant [171], assuming its eventual value for an inter-spin vacuum with units in $\left[\mathrm{N} \mathrm{m}^{2}\right]$. In the equation (6.40), it has been considered that the spins $\left(\mu_{\left|0^{A} 0^{B}\right\rangle}\right.$ and $\mu_{\left|1^{A} 1^{B}\right\rangle}$ ) are located punctually in the positions $\vec{x}\left|0^{A} 0^{B}\right\rangle$ and $\vec{x}\left|1^{A} 1^{B}\right\rangle$ as in fact happens in the Newton's equation, where the masses are considered punctual regardless of their value [179]. We will refer to the force expressed in equation (6.40) as a spinorial force. Although this force has similar characteristics to those which exist between parallel conductors in which currents flow in the same direction [198], and those existing between static electric charges of different sign (Coulomb's Law) [199], it shares the most similarities with Newton's equation [171].

On the other hand, equation (6.40) refers to the equation (3.38) of the entanglement, since if we multiply the latter by the equation of the conservation of the spin, we will have,

$$
\frac{-\mu_{\left|0^{A} 0^{B}\right\rangle} \mu_{\left|1^{A} 1^{B}\right\rangle}}{\mu_{\left|0^{A} 0^{B}\right\rangle}+\mu_{\left|1^{A} 1^{B}\right\rangle}} \mu_{\left|0^{A} 0^{B}\right\rangle}+\mu_{\left|1^{A} 1^{B}\right\rangle}=-\mu_{\left|0^{A} 0^{B}\right\rangle} \mu_{\left|1^{A} 1^{B}\right\rangle}
$$

Equation (6.41) is the heart of the equation (6.40), i.e., the latter is the result of the entanglement of the original spins in the lower part of Figure 6.14. Therefore, all entanglement would provoke an attractive force on the entangled spins. In this instance, that is, in the blue point of Figure 6.12, we would be talking about an attractive force completely independent from the mass. Surely, there must be a field associated with the spinorial force which we will call spinorial field, which suggests the existence of an entanglement field similar to the gravitational field. For example, the spinorial potential produced by $\mu_{\left|0^{A} 0^{B}\right\rangle}$ will be,

$\Phi(\vec{x})=-\frac{\xi_{o} \mu_{\left|0^{A} 0^{B}\right\rangle}}{r}=-\frac{\left.\xi_{o} \mu_{\left|0^{A} 0^{B}\right\rangle}\right\rangle}{\left.|\vec{x}-\vec{x}| 0^{A} 0^{B}\right\rangle \mid}$

Here also the acceleration will be the inverse gradient of the equation (6.42)

$\vec{a}=-\nabla \Phi$

Equation (6.43) is the Galileo's law of falling bodies, i.e., that all the bodies fall with the same acceleration in the field of equation (6.42), independently of its mass or composition, which is precisely what we have been talking since this subsection started: the independence of the mass. In this context, the principle of equivalence is also perfectly fulfilled [179].

As a consequence of what has been said so far, we also conjecture that there can be no attractive force in the blue point of Figure 6.12 without the intervention of entanglement between spins, which at that point are massless yet. The whole set of equations, but in particular equation (6.40), clearly explains why the force of gravity is so weak compared to the other forces of nature.

Now, we will explore some questions which will aim to validate the previous set of equations:

- What happens with the gravitational redshift? 
The light emitted from the surface of a star with frequency $\omega_{*}$ will arrive at a distant observer with a frequency $\omega_{\infty}$ less than $\omega_{*}[179]$. This is known as gravitational redshift. The gravitational potential on the surface of a star with a number of spins $\sum \mu$ and radius $R$ is $\Phi=-\frac{\xi_{o} \sum \mu}{R}$, being the potential in the infinite equals to zero, then the frequency will be,

$\omega_{\infty}=\left(1-\frac{\xi_{o} \sum \mu}{R c^{2}}\right) \omega_{*}$

This expression is accurate for small values of $\frac{\xi_{o} \sum \mu}{R c^{2}}$; a general relation for arbitrary values of this quotient can be obtained from GR (which of course reproduces the previous one in the limit $\left.\frac{\xi_{o} \sum \mu}{R c^{2}}<<1\right)$ and where the analysis made in this subsection is also fulfilled. The gravitational redshift predicted by the equation (6.44) has been detected in the spectrum of white dwarfs (very dense small stars with a mass comparable to that of the sun and a radius $R \sim 10^{3} \mathrm{Km}$ ), for which $\frac{\xi_{o} \sum \mu}{R c^{2}} \sim 10^{-3}$.

\section{- How is the geometry in the environment of a spherical star?}

One of the simplest solutions of Einstein's equations, yet one of the most useful, is that one obtained in an empty space around a source of spherically symmetric curvature, such as a spherical star [179]. This was obtained in 1916 by Karl Schwarzschild and is therefore called Schwarzschild's geometry. In a particular coordinate system, it is summarized in the line element as follows:

$d s^{2}=-\left(1-\frac{2 \xi_{o} \sum \mu}{r c^{2}}\right)(c d t)^{2}+\left(1-\frac{2 \xi_{o} \sum \mu}{r c^{2}}\right)^{-1} d r^{2}+r^{2}\left(d \theta^{2}+\sin ^{2} \theta d \phi^{2}\right)$

And so we can continue with all the symbiosis imaginable between everything established in GR [185] based on mass with the conjecture here based on spin and entanglement, thanks to which we can obtain several interesting conclusions:

- This can also be explained from another perspective because a massless photon deviates when it passes near a massive star (Sun) as shown in Figure 6.15. This is one of the reasons why GR replaced Newtonian mechanics, but with the equation (6.40) it is perfectly explained.

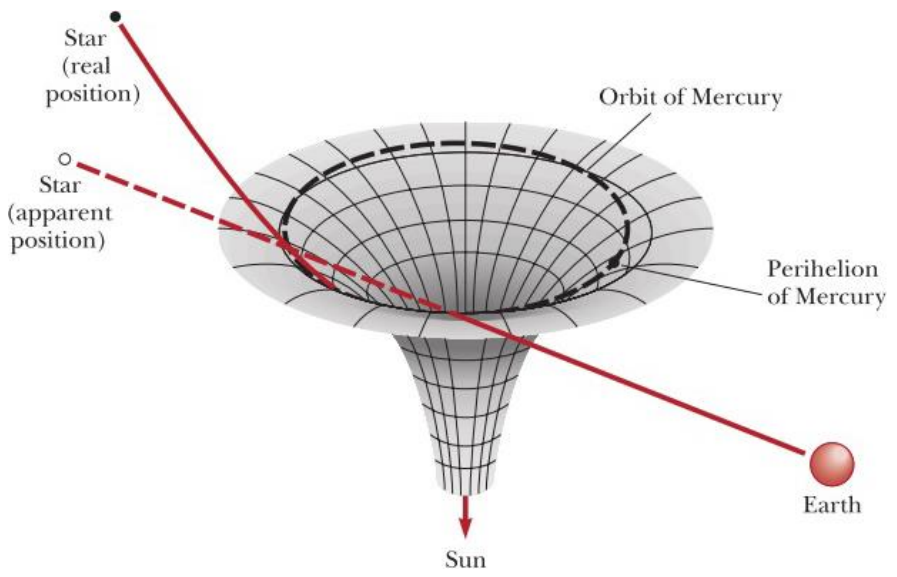

Figure 6.15 Beam of light coming from a star that deviates by action of the gravitational field of the sun, which deforms the space-time distorting the perception that is obtained on Earth from its position. 
In this case, we can adapt equation (6.40) properly,

$$
F=\xi_{o} \frac{-\mu_{\text {photon }} \sum_{\text {sun }} \mu}{r^{2}} \vec{r}
$$

where $\mu_{\text {photon }}$ is the spin of the independent photon, and $\sum_{\text {sun }} \mu$ is the summation of all the spins of the sun that are entangled with the independent photon.

- Since gravity is an instantaneous phenomenon like entanglement, it is said that it is not consistent with Special Relativity [185] because nothing can be faster than the speed of light. However, in this work, it was explained that the original entangled spins behave like avatars, and for this reason, entanglement is instantaneous without the need for superluminal signaling. Therefore, since the gravitational attraction proposed in this subsection is based on entanglement, it is perfectly consistent with Special Relativity without any kind of problem.

- This puts Newton back in the foreground because the form of his equation is absolutely transcendent, the problem is that it is based on the mass since he did not know the spins. In this new context, we can interpret mass simply as the way to represent the amount of spins.

- We could say that equation (6.40) gives GR the strength that it does not have.

- There is no acceleration without entanglement and every entanglement generates acceleration.

- The spinorial potential of equation (6.42) is identical to the rates of $\S 4.3$, which confirms the extraordinary symbiosis existing in all that has been said.

- In Table 4.3 of $\S 4.2$, we said that homothecy reaches the mass. If this is so, there would be cases of avatars with infinite masses and of opposite sign at the same point. Why do not they repel each other? If we apply the equation (6.40) to the case of avatars, we will have,

$$
F=\xi_{o} \frac{\left.-\mu_{\left.\pi^{A} \Uparrow^{B}\right\rangle} \mu_{\left.\|^{A} \Downarrow^{B}\right\rangle}\right\rangle}{r^{2}} \vec{r}=\xi_{o} \frac{(-\infty)(-\infty)}{0^{2}} \vec{r}=\infty^{4}
$$

This is the reason why they do not repel each other, since their spinorial attraction is much stronger than that due to the mass, because they are at a null distance from one another and both are infinite. What we are establishing here is that it is the entanglement of spins that is essential in matter of attraction instead of the attraction of masses due to Newton's. At the macro level, i.e., classical world, we speak of mass because it is much more practical than considering the immense quantity of intervening quantum spins.

- In Poincare's groups [84, 89], spins with infinite values automatically involve massless particles. From Einstein we know that if the particles did not have mass (as in the case of the photon), then they would travel at the speed of light. But going back to Table 4.3 of $\S 4.2$, the avatars of the entangled photons are massless, so why do not they fly out at the speed of light? We must remember from $\$ 3$ that if the entangled photons are separated from each other, then, the avatars will move accordingly but always in the middle of the original spins, i.e., as the original photons move, so will the avatars, but they will always be in the middle of the original photons. Moreover, we should remember from $\S 3$ that the avatars only have projection on the axis of spins exclusively, i.e., they will travel at the speed of light but in the space of the spins, not in space-time. Although they go at the speed of light, in reality, they do not go anywhere due to the dilatation of the locality.

- Everything said here is extendable to quarks [200], which have various intrinsic properties including electric charge, mass, color charge, and spin, as well as any other subatomic particle. 


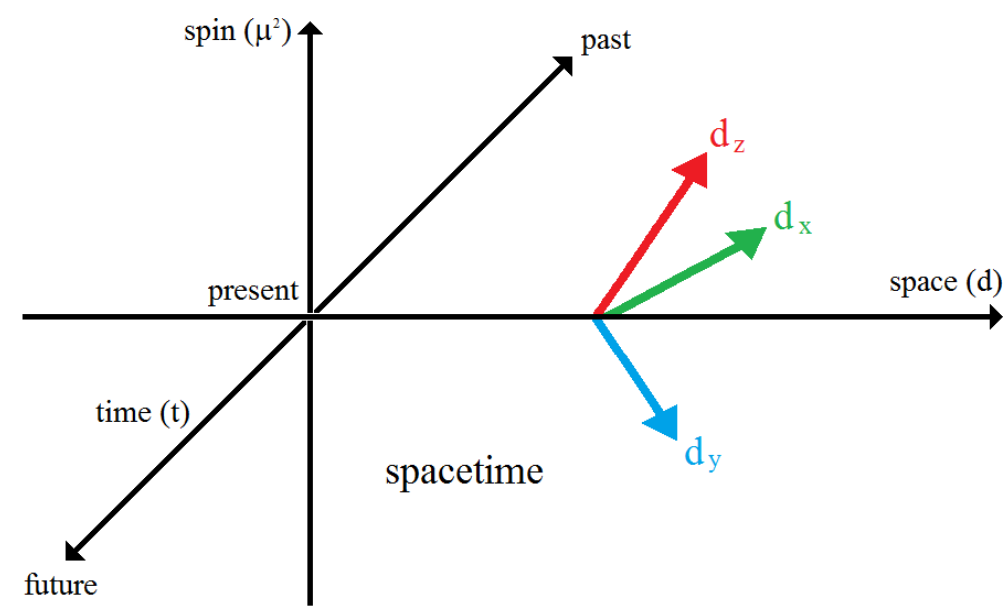

Figure 6.16 Spin space is different to space-time. The components $(\mathrm{dx}, \mathrm{dy}, \mathrm{dz}, \mathrm{t})$ of space-time do not coincide with the space of spins, which is not shown in the drawing for obvious reasons.

- Of course, we also project everything said here to the particle-anti-particle pairs as those involved in the Hawking's radiation [13].

- In Physics, the Casimir effect or the Casimir-Polder force [201] is an effect predicted by the quantum field theory that is measurable and consists of the following: given two metallic objects separated by a small distance compared to the size of the objects, an attractive force appears between both of them due to an effect associated with the quantum vacuum or vacuum energy density. In QM, vacuum energy is a kind of zero point energy existing in space even in the absence of all kinds of matter, being this absolutely consistent with everything said up to here. This attractive force: the CasimirPolder's, is nothing other than entangled pairs that pull between the plates together. In other words, the Casimir effect is a proof of what is said here and what is said here is a proof of how the Casimir effect actually works.

- What about dark matter and energy? In physical cosmology, dark energy is a form of energy [202] that would be present in all space, producing a pressure that tends to accelerate the expansion of the universe, resulting in a repulsive gravitational force [203]. We know that both dark matter and dark energy are a property of gravity [204]. Dark matter and dark energy are reflected in a) the large scale space curved structure of the universe caused by gravity, and b) the gravitational attracting and repelling aspects of gravity. We recognize three instances involved in this topic: dark matter, dark energy and inflation [205]. If we adapt the tools presented in this subsection, then we can explain all the elements involved in the Principle of Interaction Dynamics (PID) induced by the cosmological model [206]: the cosmic radius (of the 3D spherical universe), the negative pressure generated by the repulsive aspect of gravity, the dual gravitational potential, and the cosmic density. In this context, a great achievement would be to explain the expansion of the universe through the tools discussed in this subsection. For this purpose, we propose two possibilities:

a) Through the force of equation (6.40). Unfortunately, this possibility is a bit confusing, given that this force is the product of an entanglement and therefore attractive. So, how could it represent a repulsive force? In the upper part of Figure 6.14, we showed two completely independent spins that did not attract each other and we concluded that there was a kind of indifferent state due to the lack of entanglement. But, it happens that for the gravity there is no indifference, that is, there is attraction, or there is repulsion.

$$
\begin{aligned}
& F=\xi_{o} \frac{\left.-\mu_{\mid 0^{A} 0^{B}} \mu_{\left|1^{A} 1^{B}\right\rangle}\right\rangle}{r^{2}} \vec{r}\left(\mu_{\left|0^{A} 0^{B}\right\rangle}>0, \mu_{\left|1^{A} 1^{B}\right\rangle}<0, F>0\right) \text { (entanglement = attraction) } \\
& F=\xi_{o} \frac{\left.-\mu_{\left|0^{A} 0^{B}\right\rangle} \mu_{\left|1^{A} 1^{B}\right\rangle}\right\rangle}{r^{2}} \vec{r}\left(\mu_{\left|0^{A} 0^{B}\right\rangle}>0, \mu_{\left|1^{A} 1^{B}\right\rangle}>0, F<0\right) \text { (no entanglement = repulsion) }
\end{aligned}
$$


b) Perhaps, for this adventure of thought proposed by $\mathrm{GR}=\mathrm{QM}$, the universe expands from one point (Big Bang) and meets in another point (Big Crunch) as we can see in Figure 6.17. If we interpret this process as a big entanglement, then, on one side, things are separated, and on the other side, they meet since $\mathrm{BB}$ and $\mathrm{BC}$ are entangled. It is as if $\mathrm{BB}$ was the victim of a great decay. But if we remember that the avatars are only sensitive to their own space, i.e., the space of the spins, then, what in the Euclidean space is expansion, in the space of the spins is simply transit and certainly of duration equal to zero. That is, all the time of the universe is an instant for the avatars who hold this effect since they are at an intermediate point between BB and BC all together. It is as if all the matter we see is going through a huge multidimensional WH and that the time that seems immense to us, for the avatars that manage such a colossal event is instantaneous. All this happens in presence of a dilation of the locality due to avatars. Recall that at the beginning of the universe, the speed of light was $c=\infty$, that is, the entanglement with the dilatation of the locality brings us back to the origins of the universe itself. Moreover, Friedmann [192-196] considered models with matter that expanded from a singularity of infinite density to explain the expansion of the universe, which also speaks of a concentration of matter characterristic of the avatars. Finally, as we have shown in this paper, mass is not necessary for attraction and repulsion, but what is only necessary is the entanglement or its absence, respectively, then based on the above we can explain several more phenomena such as: gravitational lens and gravitational waves, among others.

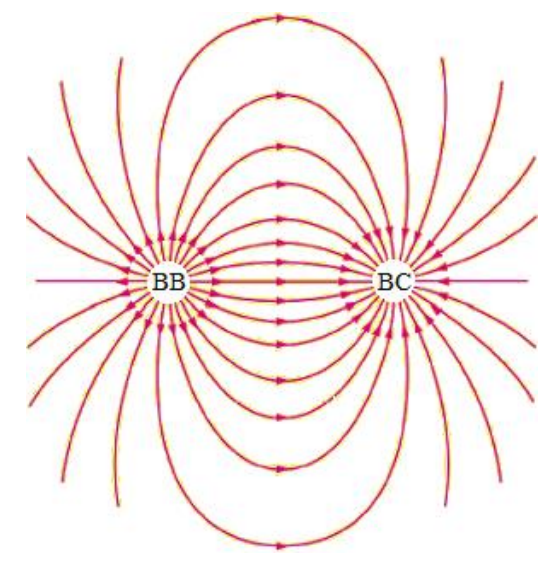

Figure 6.17 From a Big Bang to a Big Crunch.

\section{Conclusions and Future Works}

We must not lose the perspective that the most important thing is beyond evaluating the viability of the slogan GR=QM or the dissatisfaction of any result obtained through an experiment based on Bell's theorem, in fact, this research is a lifting shot above these towards our primigenius commitment which is related to quantum communications and the exceptional attributes of a link based on entanglement for that purpose. Honoring this commitment, the present section is divided into two parts: conclusions and future works, where the first part is organized by sections and finishes with a general analysis, whereas, future works represents the pending experiments that has arisen from this work.

\subsection{Conclusions}

\section{In §3, we saw that:}

- as a result of the interaction between original particles some shadows, reflexes, ghosts, spectra, alteregos, or avatars appear in the exact half of the distance that separates such original particles, although each one is in opposite places of the universe,

- the avatars are all together in that single and intermediate point,

- the ones who really communicate are the avatars, 
- the avatars are the operative representatives of the original spins, that is, it is as if the original spins behave like the avatars and thus justify the exceptional attributes of entanglement,

- the avatars of equation (3.43) were obtained from equation (3.34), i.e., the scalar version of $S^{A \cup B}$. We would have never found the avatars with the Dirac's notation based on <bra| and $|k e t\rangle$ of equation (3.31) since with that notation: values, signs and polarizations (or orientations) of spins are blurred; which is not advisable, in particular, when we try to study different degrees of correlation between them. All this is particularly important considering that the spin is the most conspicuous element of QM. In the end, everything was reduced to a problem of symbolic logic, i.e., a simple problem of mathematical treatment. For this reason, two apparently incompatible theories (GR and QM) have always been united even if we did not see it [71]. Although equation (6.2) collects both theories with traditional tools, we would have never reached it without equation (6.1), since it comes exclusively from scalar notation.

\section{In $\$ 4$, we saw that:}

- when in Table 4.3 of $\S 4.2$ we speak of negative mass, we are talking about negative matter and negative energy; where mass and energy are completely conserved, i.e., they are neutralized in the total balance. However, this tells us that the entanglement brings together the matter and antimatter (where it is) of the particles entangled through the avatars. It is as if the avatars reflect the fact that there is a counterpart of each particle entangled in another part. In a way, it is as if Alice and Bob looked at themselves in a mirror, in other words: a kind of self-entanglement between matter and antimatter. All this leads us to ask ourselves if the entanglement is the result of anti-matter almost exclusively and if without anti-matter entanglement would not even exist. This has automatic consequences on: a) the Casimir's effect [201], which being the result of the joint action of matteranti-matter pairs, would then be directly linked to entanglement, b) dark energy and matter [89, 202] is a similar case to the latter almost for the same reasons,

- in $\S 4.3$, we saw that the avatars sustain the effect called entanglement.

\section{In §5, we saw that:}

- in Figure 5.17 as a result of a quantum measurement, the avatars go from being 4 with infinite value in the case of entanglement, to being 2 with finite value in the case of completely independent particles,

- the rest of $\S 5$ talks about the extension of the avatars-based analysis to all the possibilities of entanglement.

\section{As a consequence of $\S 6$, we know that:}

- the entanglement is a hinge that connects the two most important theories of Physics (GR and QM) and not the reason for their disagreement,

- the avatars are an approach to the Theory of Everything (TOE) through the equations (6.1) and (6.2),

- the avatars have infinite energy of both signs on both sides of the WREP, which is the kind of necessary energy to keep a wormhole open. This feature is the fuel for a natural, equivalent and instantaneous transit through the WREP while the entanglement lasts,

- a complete explanation of the instantaneity of the entanglement is possible for the first time without the need of a confrontation between GR and QM. This instantaneity takes place between entangled pairs which constitute a unique state from its member states, in such a way that, when we touch something of this set, we touch everything. Both literature and experiments indicate that entanglement is a non-local effect, justifying its instantaneity at the expense of a dispute between GR and QM. Such dispute is saved, in part, by the following slogan: entanglement is instantaneous but does not allow us to transmit useful information without using superluminal signaling which collides with the postulates of the Theory of Relativity. In this work, it has been shown that this is not the case, 
- we should not confuse a link based on entanglement (LBoE) with teleportation, which is one of the many uses of an LBoE, but it is not the only one. LBoE has infinite bandwidth, null latency, and absence of noise; however, teleportation uses EPR pairs and a series of optical elements which introduce noise in said pairs. Therefore, teleportation in itself will have the bandwidth of the component with the lowest bandwidth,

- no superluminal velocities are needed to justify the instantaneity of entanglement; therefore, QM is a complete theory, since avatars can communicate at speeds well below that of light. If the original spins were the ones that communicated between themselves, then they would need an infinite speed to do so,

- non locality $=$ dilated locality,

- since gravity and entanglement share the same exceptional attributes:

a) there is not a screen that can stop them,

b) they are instantaneous, and

c) they have an infinite range;

and based on $\$ 6.5$, we concluded that there is a strong clue that leads us to say that gravity and entanglement would be expressions of a common phenomenon:

$$
(\text { Gravity }=\text { Entanglement }) \Rightarrow(\mathrm{GR}=\mathrm{QM}),
$$

where, in both cases the equal sign "=" means avatars.

\subsection{Future works}

From everything seen so far, we consider that there are pending tasks which can be carried out using the tools developed in this work, therefore, we think that the following is possible:

- that LBoE becomes unnecessary to disciplines such as Cryptography and Data Compression,

- the implementation of qubits and quantum gates based on LC circuits cooled with liquid helium to eliminate their resistive components,

- the analysis of superconductor qubits, relatives to Cooper's pairs for Josephson's junction,

- the simulation of an LBoE and a teleportation with LC circuits,

- that, if there are gravitational waves, then, there would be entanglement waves, and, if so: will they be useful to explain the double slit experiment? [211,212],

- a better analysis of positronium and anti-matter,

- the analysis of other apparently superluminal elements, e.g., the tachyons,

- the teleportation of energy with the simplified protocol of $\S 6.2$,

- the analysis of quasi-entanglement for Bio-entanglement. Since in the macroscopic nature (classical world) there are not two perfectly equal things, then we cannot speak of entanglement, but of quasientanglement,

- the analysis of an eventual relationship between entanglement and dark matter [89, 202-206], the explanation of it, the analysis of its existence, and an evaluation of whether or not it is necessary since the entanglement could do everything,

- an experiment to proof that two entangled photons attract each other,

- a trainable entanglement or quasi-entanglement, that is, to see the entanglement as a learning phenomenon. This question has always been latent: is the entanglement trainable? Can we make a supervised or unsupervised purification or distillation? In the supervised case the objective is to try to reach a target, and in the unsupervised case minimize an energetic functional. The answer to these questions constitutes a bridge between the two most important current scientific-technological disciplines: Quantum Information Processing [51-53] and Artificial Intelligence [207-209], and the so called Quantum Artificial Intelligence [210].

- the idea that entanglement can take place among particles that have never coexisted spatially. This is equivalent to the case of particles that did not emerge from a decay process, or the fact of these being in different places do not imply that a distribution existed. This eventual possibility constitutes the holy grail of the entanglement. 


\begin{abstract}
Epilogue - We believe that the only glimpse of viability of GR=QM is through such an eclectic and teratological tool as the avatars, and we also believe that these would never have arisen from the Theory of Strings which seems to be the putative territory of the creator of the slogan. After all, how else can two universes as vast as GR and QM be joined if something does not go out of range, at least for a little while?
\end{abstract}

\title{
Competing Interests
}

The author declares that there are no competing interests.

\section{Funding Statement}

The author acknowledges funding by Qubit Reset LLC under contract QComm-01\#05/01/2015.

\section{Acknowledgments}

M. Mastriani thanks boarding of Qubit Reset LLC for his tremendous help and support.

\section{References}

1. Audretsch, J. (2007) Entangled Systems: New Directions in Quantum Physics. Wiley-VCH Verlag GmbH \& Co, Weinheim.

2. Jaeger, G. (2009) Entanglement, Information, and the Interpretation of Quantum Mechanics. The Frontiers Collection. Springer-Verlag, Berlin.

3. Horodecki, R. et al. (2007) Quantum entanglement. (https://arxiv.org/abs/quant-ph/0702225)

4. Einstein, A., Lorentz, H.A., Minkowski, H., Weyl, H. (1952) The Principle of Relativity: a collection of original memoirs on the special and general theory of relativity. Courier Dover Publications. N.Y.

5. Phillips, A.C. (2003) Introduction to Quantum Mechanics. Wiley. N.Y.

6. Newton, R.G. (2002) Quantum Physics: A text for Graduate Students. Springer, N.Y.

7. Gasiorowicz, S. (2003) Quantum Physics. John Wiley and Sons, N.Y.

8. Peres, A. (2002) Quantum Theory: Concepts and Methods. Kluwer Academic Publishers, N.Y.

9. - (2009) Compendium of Quantum Physics: Concepts, Experiments, History and Philosophy. Greenberger, Daniel, Hentschel, Klaus, Weinert, Friedel (Eds.) 404-405. Springer-Verlag, Berlin.

10. Al-Khalili, J. (1999) Black Holes, Wormholes and Time Machines, CRC Press. N.Y.

11. Hawking, S.W. (1976) Black holes and thermodynamics. Physical Review D 13:2, 191-197. (https://doi.org/10.1103/PhysRevD.13.191)

12. Hawking, S.A. (1998) Brief history of time. Bantam Books. London.

13. Steinhauer, J. (2016) Observation of quantum Hawking radiation and its entanglement in an analogue black hole. Nature Physics, 12, 959-965. (https://doi.org/10.1038/nphys3863)

14. Almheiri, A., Marolf, D., Polchinski, J., Sully, J. (2012) Black Holes: Complementarity or Firewalls? (https://arxiv.org/abs/1207.3123)

15. Hawking, S. (1975) Particle Creation by Black Holes. Commun. Math. Phys. 43:3, 199-220. (https://doi.org/10.1007/BF02345020)

16. Susskind, L. (2008) The Black Hole War: My Battle with Stephen Hawking to Make the World Safe for Quantum Mechanics. Hachette Inc.

17. Maldacena, J., Susskind, L. (2013) Cool horizons for entangled black holes. (https://arxiv.org/abs/1306.0533)

18. Susskind, L. (2014) ER=EPR, GHZ, and the Consistency of Quantum Measurements. (https://arxiv.org/abs/1412.8483)

19. Susskind, L. (2016) Copenhagen vs Everett, Teleportation, and ER=EPR. (https://arxiv.org/abs/1604.02589)

20. De Aquino, F. (2012) TOE: Theory of Everything. (https://arxiv.org/abs/gr-qc/9910036)

21. Einstein, A., Podolsky, B., Rosen, N. (1935) Can Quantum-Mechanical Description of Physical Reality Be Considered Complete? Physical Review. 47:10, 777-780.

(https://doi.org/10.1103/PhysRev.47.777)

22. Busch, P., Lahti, P., Pellonpää, J.P., Ylinen, K. (2016) Quantum Measurement. Springer, N.Y. 
23. Bancal, J-D. et al. (2012) Quantum non-locality based on finite-speed causal influences leads to superluminal signaling. Nature Physics. 8, 867-870. (https://doi.org/10.1038/nphys2460)

24. Ghirardi, G.C. et al. (1988) Experiments of the EPR Type Involving CP-Violation Do not Allow Faster-than-Light Communication between Distant Observers. Europhys. Lett. 6:2, 95-100. (https://doi.org/10.1209/0295-5075/6/2/001)

25. Eberhard, P.H., Ross, R.R. (1989) Quantum field theory cannot provide faster-than-light communication. Foundations of Physics Letters. 2:2, 127-149. (https://doi.org/10.1007/BF00696109)

26. Chashchina, O.I., Silagadze, Z.K. (2012) Breaking the light speed barrier. (https://arxiv.org/abs/1112.4714)

27. Herbert, N. (1982) FLASH-A superluminal communicator based upon a new kind of quantum measurement. Foundations of Physics. 12:12, 1171-1179. (https://doi.org/10.1007/BF00729622)

28. Weinstein, S. (2006) Superluminal Signaling and Relativity. Synthese, 148:2, 381-399. (https://doi.org/10.1007/s11229-004-6231-5)

29. Weinstein, G. (2012) Einstein on the Impossibility of Superluminal Velocities. (https://arxiv.org/abs/1203.4954)

30. Bell, J. (1964) On the Einstein Podolsky Rosen paradox. Physics Physique Fizika. 1:3, 195-200. (https://doi.org/10.1103/PhysicsPhysiqueFizika.1.195)

31. Aspect, A., Grangier, P., Roger, G. (1982) Experimental Realization of Einstein-Podolsky-RosenBohm Gedankenexperiment: A New Violation of Bell's Inequalities. Physical Review Letters. 49:2, 91-94. (https://doi.org/10.1103/PhysRevLett.49.91)

32. Aspect, A., Dalibard, J., Roger, G. (1982) Experimental Test of Bell's Inequalities Using TimeVarying Analyzers. Physical Review Letters. 49:25, 1804-1807. (https://doi.org/10.1103/PhysRevLett.49.1804)

33. Bohm, D. (1952) A suggested interpretation of the Quantum Theory in terms of 'Hidden' Variables, I and II". Physical Review. 85, 166-193. (https://link.aps.org/doi/10.1103/PhysRev.85.166)

34. Clauser, J.F., Horne, M.A., Shimony, A., Holt, R.A. (1969) Proposed experiment to test local hidden-variable theories. Physical Review Letters, 23:15, 880-884. (https://doi.org/10.1103/PhysRevLett.23.880)

35. Hnilo, A.A. (2016) Dos hipótesis ocultas en las desigualdades de Bell. An. AFA. 27:3, 109-114. (http://www.scielo.org.ar/pdf/aafa/v27n3/v27n3a06.pdf)

36. Hnilo, A.A. (2017) Using Measured Values in Bell's Inequalities Entails at Least One Hypothesis in Addition to Local Realism. Entropy, 19:4, 180. (https://doi.org/10.3390/e19040180)

37. Doplicher, S. (2009) The Principle of Locality. Effectiveness, fate and challenges. (https://arxiv.org/abs/0911.5136)

38. Blaylock, G. (2009) The EPR paradox, Bell's inequality, and the question of locality. (https://arxiv.org/abs/0902.3827)

39. Aspect, A. (2007) Quantum mechanics: To be or not to be local. Nature. 446:7138, 866-867. (https://doi.org/10.1038/446866a)

40. Horodecki, M. et al. (2003) Local information as a resource in distributed quantum systems. Physical Review Letter, 90, 100402. (https://doi.org/10.1103/PhysRevLett.90.100402)

41. Popescu, S., Rohrlich, D. (1997) Causality and Nonlocality as Axioms for Quantum Mechanics. (https://arxiv.org/abs/quant-ph/9709026)

42. Wald, R.M. (2006) Resource letter TMGR-1: teaching the mathematics of general relativity. Am. J. Phys. 74, 471-477. (https://doi.org/10.1119/1.2178850)

43. Weingard, R. (1972) Relativity and the reality of past and future events. Br. J. Philos. Sci., 23:2, 119-121. (https://doi.org/10.1093/bjps/23.2.119)

44. Petkov, V. (2005) Relativity and the Nature of Space-time. Springer, Berlin. Chap. 10.

45. Rindler, W. (1977) Essential Relativity. Springer, Berlin. 244.

46. Synge, J. L. (1960) Relativity: The General Theory. Nord-Holand, Amsterdam. 109.

47. Hanson, R. (2015) Loophole-free Bell inequality violation using electron spins separated by 1.3 kilometres. Nature. 526: 682-686. (https://doi.org/10.1038/nature15759)

48. Bell, J.S. (2004) Speakable and unspeakable in quantum mechanics, Cambridge, 52-62. 
49. Tittel, W. et al (1998) Experimental demonstration of quantum correlations over more than 10 km. Physical Review A, 57:5, 3229-3232.

50. Abellán, C., et al (2018) Challenging local realism with human choices: The BIG Bell Test Collaboration. Nature, 557, 212-216

51. Nielsen, M.A., Chuang, I.L. (2004) Quantum Computation and Quantum Information. Cambridge University Press, Cambridge.

52. Kaye, P., Laflamme, R., Mosca, M. (2004) An Introduction to Quantum Computing. Oxford University Press, Oxford.

53. Stolze, J., Suter, D. (2007) Quantum Computing: A Short Course from Theory to Experiment. WILEY-VCH Verlag GmbH \& Co. KGaA, Weinheim.

54. Dieks, D. (1982) Communication by EPR devices. Physics Letters A, 92:6, 271-272. (https://doi.org/10.1016/0375-9601(82)90084-6)

55. Bouwmeester, B.D. et al. (1998) Experimental quantum teleportation, Phil. Trans. R. Soc. Lond. A, 356, 1733-1737. (https://doi.org/10.1098/rsta.1998.0245)

56. Bennett, C.H. et al. (1993) Teleporting an Unknown Quantum State via Dual Classical and Einstein-Podolsky-Rosen Channels. Phys. Rev. Lett. 70, 1895. (https://doi.org/10.1103/PhysRevLett.70.1895)

57. Bouwmeester, D. et al. (1997) Experimental Quantum Teleportation. Nature, 390, 575-579. (https://doi.org/10.1038/37539)

58. Boschi, D. et al. (1998) Experimental Realization of Teleporting an Unknown Pure Quantum State via Dual Classical and Einstein-Podolsky-Rosen Channels. Phys. Rev. Lett., 80, 1121. (https://doi.org/10.1103/PhysRevLett.80.1121)

59. Kurucz, Z., Koniorczyk, Z., Janszky, J. (2001) Teleportation with partially entangled states. Fortschr. Phys. 49,:10-11, 1019-1025. (https://doi.org/10.1002/1521-3978(200110)49:10/11<1019::AID-PROP1019>3.0.CO;2-Z)

60. Yu, X.-T., Zhang, Z.-C., Xu J. (2014) Distributed wireless quantum communication networks with partially entangled pairs. Chin. Phys. B, 23:1, 010303. (https://doi.org/10.1088/1674-1056/23/1/010303)

61. MacKay, D.J.C. (2003) Information Theory, Inference, and Learning Algorithms Cambridge University Press.

62. NIST (2014) Quantum Computing and Communication. CreateSpace Independent Publishing Platform.

63. Pathak, A. (2013) Elements of Quantum Computation and Quantum Communication. Taylor \& Francis Group, Boca Raton.

64. Anderson, R., Brady, R. (2013) Why quantum computing is hard - and quantum cryptography is not probably secure. (https://arxiv.org/abs/1301.7351)

65. Cariolaro, G. (2015) Quantum Communications. Springer International Publishing. N.Y.

66. Mishra, V.K. (2016) An Introduction to Quantum Communication. Momentum Press, N.Y.

67. Imre, S., Gyongyosi, L. (2012) Advanced Quantum Communications: An Engineering Approach. Wiley-IEEE Press, N.Y.

68. Hadamard, J. (2008) LESSONS IN GEOMETRY: I. Plane Geometry. American Mathematical Society, Providence, R.I.

69. Meserve, B.E. (1955) Homothetic transformations: Fundamental Concepts of Geometry, Addison -Wesley, 166-169.

70. Tuller, A. (1967) A Modern Introduction to Geometries, University Series in Undergraduate Mathematics, Princeton, N.J.: D. Van Nostrand Co.

71. Susskind, L. (2017) Dear Qubitzers, GR=QM. (https://arxiv.org/abs/1708.03040)

72. Karris, S.T. (2004) Circuit Analysis I with MATLAB ${ }^{\circledR}$ Applications. Orchard Publications, Fremont, California.

73. Alexander, C.K., Sadiku, M.N.O. (2007) Fundamentals of Electric Circuits. McGraw-Hill, N.Y.

74. Malacara, D., Malacara, Z. (2004) Handbook of Optical Design, Marcel Dekker Inc, N.Y.

75. Hecht, E. (2017) Optics, Pearson, Essex.

76. Huggins, E.R. (2000) Physics 2000: Geometrical Optics. Moose Mountain Digital Press, New Hampshire.

77. Benjamin Crowell, B. (1999) Optics. Light and Matter. Fullerton, California. 
78. Cramer, J., Witek, B., Zwiller, V. (2011) Violation of Bell's Inequality: Manual for the Quantum Entanglement Setup. Zwiller Lab, TUDelft. (http://zwillerlab.tudelft.nl/wp-content/uploads/2011/04/manual_entanglement.pdf)

79. Edgren, L., Marnelius, R., Salomonson, P. (2005) Infinite spin particles. (https://arxiv.org/abs/hep-th/0503136)

80. Longo, R., Morinelli, V., Rehren, K-H. (2016) Where Infinite Spin Particles are Localizable. Commun. Math. Phys. 345, 587-614. (https://doi.org/10.1007/s00220-015-2475-9)

81. Zinoviev, Y.M. (2017) Infinite Spin Fields in $d=3$ and Beyond, MDPI Universe, 3, 63. (https://doi.org/10.3390/universe3030063)

82. Bengtsson, A. K. H. (2014) BRST Theory for Continuous Spin. (https://arxiv.org/abs/1303.3799)

83. Bekaert, X., Skvortsov, E. (2017) Elementary particles with continuous spin. (https://arxiv.org/abs/1708.01030)

84. Schroer, B. (2017) Wigner's infinite spin representations and inert matter. Eur. Phys. J. C 77:362. (https://doi.org/10.1140/epjc/s10052-017-4903-9)

85. Porrati, M. (2008) Universal Limits on Massless High-Spin Particles. (https://128.84.21.199/abs/0804.4672v2)

86. Baskal, S., Kim, Y.S. (1997) Little groups and Maxwell-type tensors for massive and massless particles. Europhys. Lett., 40:4, 375-380. (https://doi.org/10.1209/ep1/11997-00474-0)

87. Schuster, P., Toro, N. (2013) A Gauge Field Theory of Continuous-Spin Particles. (https://arxiv.org/abs/1302.3225)

88. Schuster, P., Toro, N. (2014) A CSP Field Theory with Helicity Correspondence. (https://arxiv.org/abs/1404.0675)

89. Schroer, B. (2014) Dark matter and Wigner's third positive energy representation class. (https://arxiv.org/abs/1306.3876)

90. Fernando, S., Günaydin, M. (2016) Massless conformal fields, AdS(d+1)/CFTd higher spin algebras and their deformations. Nuclear Physics B, 904, 494-526. (https://doi.org/10.1016/j.nuclphysb.2016.01.024)

91. Pashnev, A., Tsulaia, M. (1997) Dimensional reduction and BRST approach to the description of a Regge trajectory. (https://arxiv.org/abs/hep-th/9703010)

92. Abbott, L.F. (1976) Massless particles with continuous spin indices. Physical Review D, 13:8, 2291-2294. (https://doi.org/10.1103/PhysRevD.13.2291)

93. Brink, L. (2002) Continuous Spin Representations of the Poincare and Super-Poincare Groups. (https://arxiv.org/abs/hep-th/0205145)

94. Schuster, P., Toro, N. (2013) On the Theory of Continuous-Spin Particles: Helicity Correspondence in Radiation and Forces. (https://arxiv.org/abs/1302.1577)

95. Külske, C., Orlandi, E. (2006) A simple fluctuation lower bound for a disordered massless random continuous spin model in $\mathrm{d}=2$. (https://arxiv.org/abs/math/0604068)

96. Hirata, K. (1977) Quantization of Massless Fields with Continuous Spin. Progress of Theoretical Physics, 58:2, 652-666. (https://doi.org/10.1143/PTP.58.652)

97. Ünsal, M. (2008) Topological symmetry, spin liquids and CFT duals of Polyakov model with massless fermions. (https://arxiv.org/abs/0804.4664)

98. Didenko, V.E. Skvortsov, E.D. (2015) Elements of Vasiliev theory. (https://arxiv.org/abs/1401.2975)

99. Peach, A. (2013) MSc Particles, Strings \& Cosmology: Dissertation Higher-Spin Gauge Theories, Vasiliev Theory and Holography, Durham University.

100. Boulanger, N. et al. (2016) Higher spin interactions in four-dimensions: Vasiliev versus Fronsdal. J. Phys. A: Math. Theor. 49, 095402, 52. (https://doi.org/10.1088/1751-8113/49/9/095402)

101. Moradi, H. (2012) Higher-Spin Holographic Dualities and W-Algebras, Master's Thesis Faculty of Science, University of Copenhagen.

102. Kessel, P. (2016) The Physics of Higher-Spin Theories, Ph.D. Thesis, Humboldt-Universität zu Berlin.

103. Lepage-Jutier, A. (2014) Black Holes and Toy Cosmologies in Higher Spin Gravity, Ph.D. Thesis, McGill University.

104. Bauke, H. et al. (2014) What is the relativistic spin operator? (https://arxiv.org/abs/1303.3862) 
105. Börner, G., Ehlers, J., Rudolph, E. (1975) Relativistic Spin Precession in Two-body Systems. Astro. \& Astrophys. 44, 417-420. (http://hdl.handle.net/11858/00-001M-0000-0013-5ED0-C)

106. Weder, R.A. (1974) Spectral properties of one-body relativistic spin-zero hamiltonians. Ann. Inst. Henri Poincaré, XX:2, 211-220. (http://eudml.org/doc/75804)

107. Polyzou, W.N., Glöckle, W., Witala, H. (2012) Spin in relativistic quantum theory. (https://arxiv.org/abs/1208.5840)

108. Hamdan, N., Chamaa, A., López-Bonilla, J. (2008) On the Relativistic Concept of the Dirac's electron spin. Lat. Am. J. Phys. Educ. 2:1, 65-70. (http://www.lajpe.org/jan08/LAJPE_126_Hamdan_Bonilla.pdf)

109. Koelling, D.D., Harmon, B.N. (1977) A technique for relativistic spin-polarised calculations? J. Phys. C: Solid State Phys., 10, 3107-3114. (https://doi.org/10.1088/0022-3719/10/16/019)

110. Feshbach, H., Villars, F. (1958) Elementary Relativistic Wave Mechanics of Spin 0 and Spin 1/2 Particles. Reviews of Modern Physics, 30:1, 24-45. (https://doi.org/10.1103/RevModPhys.30.24)

111. Choi, T. (2013) Relativistic Spin Operator and Lorentz Transformation of the Spin State of a Massive Dirac Particle. Journal of the Korean Physical Society, 62:8, 1085-1092. (https://doi.org/10.3938/jkps.62.1085)

112. McKenzie, C-A. (2015) An Interpretation of Relativistic Spin Entanglement Using Geometric Algebra. Electronic Theses and Dissertations. 5652.

113. Alsing, P.M., Milburn, G.J. (2002) Lorentz Invariance of Entanglement. (https://arxiv.org/abs/quant-ph/0203051)

114. Ahn, D., Lee, H-J., Hwang, S.W. (2002) Relativistic entanglement of quantum states and nonlocality of Einstein-Podolsky-Rosen (EPR) paradox. (https://arxiv.org/abs/quant-ph/0207018)

115. Alba, D., Crater, H.W., Lusanna, L. (2010) Relativistic Quantum Mechanics in the Rest-Frame Instant Form of Dynamics. (https://arxiv.org/abs/0907.1816)

116. Friis, N. et al. (2010) Relativistic entanglement of two massive particles. (https://arxiv.org/abs/0912.4863)

117. Lusanna, L. (2010) Relativistic Entanglement from Relativistic Quantum Mechanics in the RestFrame Instant Form of Dynamics. (https://arxiv.org/abs/1012.3374)

118. Crater, H.W., Lusanna, L. (2014) On Relativistic Entanglement and Localization of Particles and on their Comparison with the Non-Relativistic Theory. (https://arxiv.org/abs/1306.6524)

119. Horwitz, L., Arshansky, R.I. (2017) Relativistic Entanglement. (https://arxiv.org/abs/1707.03294)

120. Barrett, J.A. (2015) Entanglement and Disentanglement in Relativistic Quantum Mechanics, Studies in History and Philosophy of Science Part B: Studies in History and Philosophy of Modern Physics, 48, 168-174. (https//doi.org/10.1016/j.shpsb.2014.08.004)

121. Palge, V. (2013) Relativistic entanglement of single and two particle systems, Ph.D. Thesis, University of Leeds.

122. Dunningham, J., Vedral, V. (2009) Entanglement and nonlocality of a single relativistic particle. (https://arxiv.org/abs/0901.0844)

123. Palge, V., et al (2018) Relativistic entanglement of two particles driven by continuous product momenta. (https://arxiv.org/abs/1409.1316)

124. Lusanna, L. (2011) Relativistic entanglement from relativistic quantum mechanics in the restframe instant form of dynamics. 5th International Workshop DICE2010, Journal of Physics: Conference Series 306, 012039. (https://doi.org/10.1088/1742-6596/306/1/012039)

125. Jafarizadeh, M.A., Mahdian, M. (2011) Quantifying entanglement of two relativistic particles using optimal entanglement witness. Quantum Inf Process, 10:501-518. (https://doi.org/10.1007/s11128-010-0206-x)

126. Zych, M. et al. (2011) Quantum interferometric visibility as a witness of general relativistic proper time. (https://arxiv.org/abs/1105.4531)

127. Pikovski, I. et al. (2015) Universal decoherence due to gravitational time dilation. (https://arxiv.org/abs/1311.1095)

128. Ruiza, E.C., Giacominia, F., Bruknera, C. (2017) Entanglement of quantum clocks through gravity. PNAS, E2303-E2309. (https://doi.org/10.1073/pnas.1616427114)

129. Anastopoulos, C., Hu, B.L. (2014) Problems with the Newton-Schrödinger equations. New Journal of Physics 16, 085007. (https://doi.org/10.1088/1367-2630/16/8/085007)

130. Smerlak, M., Rovelli, C. (2007) Relational EPR. (https://arxiv.org/abs/quant-ph/0604064v3) 
131. Hamel, J.S. (2009) Relativity as a Consequence of Quantum Entanglement: A Quantum Logic Gate Space Model for the Universe. (https://arxiv.org/abs/0905.1119)

132. Friis, N. (2010) Relativistic Effects in Quantum Entanglement. (https://arxiv.org/abs/1003.1874)

133. Nikolic, H. (2012) EPR before EPR: a 1930 Einstein-Bohr thought experiment revisited. (https://arxiv.org/abs/1203.1139)

134. Cowen, R. (2015) The quantum source of space-time. Nature News. 527, $290-293$. (https://doi.org/10.1038/527290a)

135. Roston, G. B. et al (2005) Quantum entanglement, spin-1/2 and the Stern-Gerlach experiment. Eur. J. Phys. 26, 657-672. (https://doi.org/10.1088/0143-0807/26/4/012)

136. Castro Ruiz, E., Giacominia, F., Brukner, C. (2017) Entanglement of quantum clocks through gravity. PNAS, E2303-E2309. (https://doi.org/10.1073/pnas.1616427114)

137. Deng, D.L., Li, X., Das Sarma, S. (2017) Quantum Entanglement in Neural Network States. Physical Review X 7, 021021. (https://doi.org/10.1103/PhysRevX.7.021021)

138. Schlosshauer, M. (2005) Decoherence, the measurement problem, and interpretations of quantum mechanics. Reviews of Modern Physics. 76:4, 1267-1305.

(https://doi.org/ 10.1103/RevModPhys.76.1267)

139. Megidish, E. et al. (2012) Entanglement Between Photons that have Never Coexisted. (https://arxiv.org/abs/1209.4191)

140. Sackett, C.A., et al. (2000) Experimental entanglement of four particles. Nature, 404, 256-259. (https://doi.org/10.1038/35005011)

141. Barreiro, J.T., Kwiat, P.G. (2008) Hyperentanglement for advanced quantum communication. Quantum Communications and Quantum Imaging VI, edited by Ronald E. Meyers, Yanhua Shih, Keith S. Deacon, Proc. of SPIE, 7092, 70920P. (https://doi.org/10.1117/12.793513)

142. Deng, F.G., Ren, B.C., Li, X.H. (2017) Quantum hyperentanglement and its applications in quantum information processing. (https://arxiv.org/abs/1610.09896)

143. Krenna, M. et al. (2014) Generation and confirmation of a $(100 \times 100)$-dimensional entangled quantum system. PNAS, 111:17, 6243-6247. (https://doi.org/10.1073/pnas.1402365111)

144. Lanzagorta, M. (2012) Quantum Radar, Morgan \& Claypool Publishers, N.Y.

145. Eldar, Y.C. (2001) Quantum Signal Processing. Doctoral Thesis, MIT.

146. Eldar, Y.C. (2002) Oppenheim, A.V.: Quantum Signal Processing. Signal Process. Mag. 19, 12 32. (https://doi.org/10.1109/MSP.2002.1043298)

147. Cai, X.-F., Yu, X.-T., Shi, L.-H., Zhang, Z.-C. (2014) Partially entangled state bridge in quantum teleportation. Springer Front. Phys., 9:5, 646-651. (https://doi.org/10.1007/s11467-014-0432-2)

148. Li, W.-D., Zhang, J.-L., Gu, Y.-J. (2006) Quantum circuits for realizing deterministic and exact teleportation via two partially entangled pairs particles. IOP Chinese Physics, 5:3. (https://doi.org/10.1088/1009-1963/15/3/005)

149. Fuwa, M. et al. (2015) Experimental proof of nonlocal wave-function collapse for a single particle using homodyne measurements. Nature Communications. 6:6665. (https://doi.org/10.1038/ncomms7665)

150. Horodecki, P.L., Horodecki, R. (2001) Distillation and Bound Entanglement, Quantum Information and Computation, Rinton Press, 1:1, 45-75. (http://www.rintonpress.com/journals/qic-1-1/distill.pdf)

151. Kalb, N., et al. (2017) Entanglement Distillation between Solid-State Quantum Network Nodes. (https://arxiv.org/abs/1703.03244)

152. Briegel, H. J., et al. (2000) Entanglement Purification. In: Bouwmeester D., Ekert A., Zeilinger A. (eds) The Physics of Quantum Information. Springer, Berlin, Heidelberg.

153. Parker, S., Bose, S., Plenio M.B. (2003) Entanglement Purification via Entanglement Swapping. In: Braunstein S.L., Pati A.K. Eds. Quantum Information with Continuous Variables. Springer, Dordrecht.

154. Dür, W., Briegel, Hans.-J. (2006) Purification and Distillation, in Lectures on Quantum Information. D. Bruß and G. Leuchs Eds. Wiley-VCH Verlag GmbH, Weinheim, Germany.

155. Dür, W., Briegel, H.J. (2007) Entanglement purification and quantum error correction. (https://arxiv.org/abs/0705.4165)

156. Kiktenko, E.O., Popov, A.A. and Fedorov, A.K. (2016) Bidirectional imperfect quantum teleporttation with a single Bell state (https://arxiv.org/abs/1602.01420) 
157. Metcalf, H.J., van der Straten, P. (2003) Laser Cooling and Trapping. Journal of the Optical Society of America B. 20. (https://doi.org/10.1364/JOSAB.20.000887)

158. Vuletic, V., Chu, S. (2000) Laser Cooling of Atoms, Ions, or Molecules by Coherent Scattering. Physical Review Letters. 84:17 (https://doi.org/10.1103/PhysRevLett.84.3787)

159. Morris, M.S., Thorne, K.S. (1988) Wormholes in space-time and their use for interstellar travel: A tool for teaching general relativity. American Journal of Physics, 56:5, 395-412. (https://doi.org/10.1119/1.15620)

160. Chena, P., Wua, C-H., Yeomaz, D-h. (2017) Broken bridges: A counter-example of the ER=EPR conjecture. (https://arxiv.org/abs/1608.08695)

161. Gao, P., Jafferis, D.L., Wall, A. C. (2017) Traversable Wormholes via a Double Trace Deformation. (https://arxiv.org/abs/1608.05687)

162. Maldacena, J., Stanford, D., Yang, Z. (2017) Diving into traversable wormholes. (https://arxiv.org/abs/1704.05333)

163. Susskind, L., Zhao, Y. (2017) Teleportation Through the Wormhole. (https://arxiv.org/abs/1707.04354)

164. Nandi, K.K., Zhangb, Y-Z., Kumar, K.B.V. (2004) Semiclassical and Quantum Field Theoretic bounds for traversable Lorentzian Stringy Wormholes. (https://arxiv.org/abs/gr-qc/0407032)

165. Mastriani, M. (2018) Simplified Protocol of Quantum Teleportation. <hal-01833706v3>

166. Wootters, W.K., Zurek, W.H. (1982) A single quantum cannot be cloned. Nature, 299, 802-803. (https://doi.org/10.1038/299802a0)

167. Zhong-Xiao M., et al. (2015) Cavity-based architecture to preserve quantum coherence and entanglement. Nature Scientific Reports 5, 13843. (https://doi.org/10.1038/srep13843)

168. .- (2005) CRAF Handbook for Radio Astronomy. Edited by Cohen, J., Spoelstra, T., Ambrosini, R. van Driel, W., European Science Foundation.

169. Milligan, T.A. (2005) Modern antenna design. IEEE Press. Hoboken, N.J.

170. Moreira, A. et al (2013) A Tutorial on Synthetic Aperture Radar. IEEE Geoscience and remote sensing magazine. March, 6-43.

(http://www2.geog.ucl.ac.uk/ mdisney/teaching/PPRS/PPRS_7/esa_sar_tutorial.pdf)

171. Crowell, B. (2006) Newtonian Physics. Lightandmatter, Fullerton, California.

172. ATLAS Collaboration (2018) Observation of Higgs boson production in association with a top quark pair at the LHC with the ATLAS detector. (https://arxiv.org/abs/1806.00425)

173. Ellisa, J., Murphyc, C.W., Sanz, V., You, T. (2018) Updated Global SMEFT Fit to Higgs, Diboson and Electroweak Data. (https://arxiv.org/abs/1803.03252)

174. CMS Collaboration (2018) Measurements of properties of the Higgs boson decaying to a W boson pair in pp collisions at Vs $=13 \mathrm{TeV}$. (https://arxiv.org/abs/1806.05246)

175. Kima, J.H., Sakaki, Y., Son, M. (2018) Combined analysis of double Higgs production via gluon fusion at the HL-LHC in the effective field theory approach. (https://arxiv.org/abs/1801.06093)

176. Rubio, J. (2018) Higgs inflation. (https://arxiv.org/abs/1807.02376)

177. Jana, S., Nandia, S. (2018) New Physics Scale from Higgs Observables with Effective Dimension-6 Operators. (https://arxiv.org/abs/1710.00619)

178. Athron, P., et al (2018) Impact of vacuum stability, perturbativity and XENON1T on global fits of Z2 and Z3 scalar singlet dark matter. (https://arxiv.org/abs/1806.11281)

179. Cannas, S.A., Zamar, R. (2016) Relatividad General y Cosmología: Física Contemporánea. Notas de cátedra. FAMAF, Universidad Nacional de Córdoba.

(http://www.famaf.unc.edu.ar/wp-content/uploads/2014/07/notas-relatividad1.pdf)

180. Debono, I. Smoot, G.F. (2016) General Relativity and Cosmology: Unsolved Questions and Future Directions. (https://arxiv.org/abs/1609.09781)

181. Olesen, P. (2008) General Relativity and Cosmology. Lecture notes. The Niels Bohr Institute. (http://www.nbi.dk/ polesen/GRC.pdf)

182. Pützfel, D. (2004) Introduction to General Relativity and Cosmology. Iowa State University. (http://puetzfeld.org/files/lecture1.pdf)

183. Collett, T.E. (2018) A precise extragalactic test of General Relativity. Science, 360:6395, 13421346. (https://doiu.org/10.1126/science.aao2469)

184. Saravanan, S.K. (2016) Spin Dynamics in General Relativity. Ph.D. Thesis, Lorentz Institute for Theoretical Physics, Leiden University. 
185. Birks, T. (2016) General relativity without tensors. PH30101 General Relativity. University of Bath. (http://people.bath.ac.uk/pystab/PH30101_notes.pdf)

186. Carroll, S.M. (1997) Lecture Notes on General Relativity. (https://arxiv.org/abs/gr-qc/9712019v1)

187. Hobson, M.P., Efstathiou, G.P., Lasenby, A.N. (2006) General Relativity: An Introduction for Physicists. Cambridge University Press.

188. Lambourne, R.J.A. (2011) Relativity, Gravitation and Cosmology. Cambridge University Press.

189. Blau, M. (2016) Lecture Notes on General Relativity. University of Bern. (http://www.blau.itp.unibe.ch/Lecturenotes.html)

190. Wald, R.M. (1984) General Relativity. The University of Chicago Press.

191. Weinberg, S. (1972) Gravitation and Cosmology: Principles and Applications of the General Theory of Relativity. Wiley, N.Y.

192. Friedman, A. (1922) Über die Krümmung des Raumes. Zeitschrift für Physik, 10:1, 377-386. (https://doi.org/10.1007/BF01332580)

193. Friedman, A. (1924) Über die Möglichkeit einer Welt mit konstanter negativer Krümmung des Raumes. Zeitschrift für Physik, 21:1, 326-332. (https://doi.org/10.1007/BF01328280)

194. Ryden, B.S. (2003) Introduction to Cosmology. San Francisco: Addison-Wesley

195. Tropp, E.A. (2006) Alexander A. Friedmann: The Man Who Made the Universe Expand. Cambridge: Cambridge University Press.

196. Nemiroff, R.J., Patla, B. (2007) Adventures in Friedmann Cosmology: An Educationally Detailed Expansion of the Cosmological Friedmann Equations. (https://arxiv.org/abs/astro-ph/0703739)

197. Einstein, A., Rose, N. (1935) The Particle Problem in the General Theory of Relativity. Physical Review. 48:1, 73-77. (https://doi.org/10.1103/PhysRev.48.73)

198. Coey, J.M.D. (2009) Magnetism and Magnetic Materials. Cambridge University Press.

199. Jonassen, N. (2002) Electrostatics. Springer, Boston, MA. (https://doi.org/10.1007/978-1-4615-1073-4)

200. Smirnov, B.M. (2018) Atomic Particles and Atom Systems: Data for Properties of Atomic Objects and Processes. Springer. N.Y.

201. Milton, K.A. (2001) The Casimir Effect: Physical Manifestations of Zero-Point Energy. World Scientic. N.J.

202. - (2011) Dark Matter and Dark Energy: A Challenge for Modern Cosmology. Matarrese, S., Colpi, M., Gorini, V., Moschella, U. (Eds.), Springer, N-Y.

203. Peebles, P.J.E., Ratra, B. (2003) The Cosmological Constant and Dark Energy. Reviews of Modern Physics, 75:2, 559-606. (https://doi.org/10.1103/RevModPhys.75.559)

204. - (2007) The Invisible Universe: Dark Matter and Dark Energy. Lefteris Papantonopoulos (Eds.). Springer, Berlin, Heidelberg. (https://doi.org/10.1007/978-3-540-71013-4)

205. - (2011) Dark Matter and Dark Energy: A Challenge for Modern Cosmology. Matarrese, S., Colpi, M., Gorini, V., Moschella U. (Eds.). Springer, Berlin, Heidelberg. (https://doi.org/10.1007/978-90-481-8685-3)

206. Hernandez, M., Ma, T., Wang, S. (2015) Theory of Dark Energy and Dark Matter. J. Math. Study, 48:3, 199-221. (https://doi.org/10.4208/jms.v48n3.15.01)

207. Winston, P.H. (1992) Artificial Intelligence. Addison-Wesley Publishing Company, N.Y.

208. Poole, D.L., Mackworth, A.K. (2017) Python code for Artificial Intelligence: Foundations of Computational Agents. Cambridge University Press.

209. Rich, E., Knight, K., Nair, S.B. (2009) Artificial Intelligence. McGraw-Hill, New Delhi.

210. Wichert, A. (2014) Principles of Quantum Artificial Intelligence. World Scientific Publishing, N.J.

211. Kenmoku, M, Kume, K. (2011) Young's Double Slit Experiment in Quantum Field Theory. (https://arxiv.org/abs/1103.0100v1)

212. Samuela, J. (2018) Gravity and decoherence: the double slit experiment revisited. (https://arxiv.org/abs/1706.04401v2) 